\title{
Wing in the loop: Integrating the wing into the dynamics of insect flight
}

A Dissertation
Presented to
the Faculty of the School of Engineering and Applied Science
University of Virginia
In Partial Fulfillment
of the requirements for the Degree
Doctor of Philosophy (Mechanical and Aerospace Engineering)
by
Samane Zeyghami
May 2016


(C) 2016 Samane Zeyghami 


\section{Approval Sheet}

This dissertation is submitted in partial fulfillment of the requirements for the degree of Doctor of Philosophy (Mechanical and Aerospace Engineering)

\section{Samane Zeyghami}

This dissertation has been read and approved by the Examining Committee:

Haibo Dong, Advisor

Hossein Haj-Hariri, Committee Chair

Carl Knospe

Silvia Blemker

Gavin Garner

Accepted for the School of Engineering and Applied Science:

Craig Benson, Dean, School of Engineering and Applied Science

May 2016 
To my family. 


\section{Abstract}

Insects were the first animals to evolve active flight and remain unsurpassed in many features of flight dynamics and performance. The capacity to move in 3D space provided them with superior abilities in predator-prey interactions and in exploring novel habitats. Among insects, we find animals capable of precise hovering, taking off backwards, flying sideways and landing upside down. Some insects are capable of generating forces many times their body weight. Others perform intricate mating rituals in flight. The outstanding flight capabilities of insects has intrigued scientists for centuries. However, only recently, with the advent of high speed videography and accurate tracking techniques, have researchers been able to appreciate the elegant motion of the wings and body of insects in flight. Over the past few decades, researchers have explored insect flight, searching for the clues to these unique flight capabilities. Much progress has and is being made toward understanding the unsteady aerodynamics of flapping flight. Yet many aspects of how insects maneuver and control their flight is still poorly understood.

The capacity to control and alter the flight is as essential for aerial locomotion as the generation of aerodynamic force. To control their flight, insects alter the motion of their rapidly beating wings. The wings of insects with high flapping frequencies, such as fruit flies, move more than 10 times faster than their bodies. For other insects, such as dragonflies and damselflies, this number is only 3-5 times. Currently, it is not known how the time scales of the wing and body motions are related and how the frequency of flapping affects the dynamics and control of flight. Toward addressing these questions, this work is specifically aimed at investigating the dynamics of the insect wing and integrating it into the open-loop dynamics of flight.

In this work, we focused on low flapping frequency insect flight, where the time scales of the wing and body motions are close. The pursuit of our goal is carried out via two different branches. First, we gathered accurate data on the free flight of several species of insects during different aerial maneuvers. Quantitative analysis of the flight behavior was then conducted and comparisons were made with maneuvering flight of high flapping frequency insects. In the second branch of this work, we developed a physics-based model of the insect wing and the mechanical properties of its hinge, and combined it with a model of the unsteady aerodynamics of flapping flight to study actuation of the 
wing pitch. Generation of the aerodynamic force is sensitive to the wing pitch and its dynamics and therefore this motion plays a unique role in executing aerial maneuvers. Quantitative investigations of the wing dynamics were then carried out by varying the wing geometry, kinematics and structural properties. The ratio of the aerodynamic to elastic force, termed as Cauchy number, was identified as the most important parameter in governing wing motion. The analysis of the wing dynamics also revealed a mechanism by which the motions of the wing and body are coupled together. During aerial maneuvers, the body motion alters the balance of forces on the wing, causing the wing kinematics to passively change in response. Furthermore, the changes in the wing kinematics results in altering the force and consequently the body motion. This chain of actions and reactions is the source of coupling in the dynamics of the wing and the body. This phenomenon, which was predicted by our theoretical model and verified by our experimental measurements, cannot be explained by the currently accepted model of the insect flight dynamics. The modifications that were made to the current model based on our analysis and findings resulted in an improved model of the insect flight dynamics that can successfully predict the connection between the wing and body motions during aerial maneuvers. 


\section{Acknowledgements}

Five years ago, I started my $\mathrm{PhD}$ studies wanting to learn how to comprehend and interpret the world around me. Today, I am even more eager to continue learning and I owe that to the great experience I had working on my research. I learned a great deal from my predecessors and colleges in this field and I owe my incremental contribution to all whom came before me and enlightened the path. I am especially very thankful of my advisor, Haibo Dong, who has supported me intellectually, critically and financially throughout these years. He gave me the freedom to explore and has always encouraged me and believed in me, even when I lost my faith. I would like to thank my committee, Hossein Haj-Hariri, Silvia Blemker, Carl Knospe and Gavin Garner, for their comments and guidance throughout this research. I am also grateful for all the help and support that I have received from the faculty, staff and students in Department of Mechanical and Aerospace Engineering here at UVa. I want to also thank my previous advisor, M. Malaek, who had a great impact on shaping my scientific personality. As I worked on different projects during these past years, there were several of my teammates that helped me with gathering and reconstructing insect flight videos, with discussions and constructive criticism and with editing my writing. I would like to thank them all; Ayodeji, Chengyu, Geng, Yan, Nidhin and John. In addition, I could not have got through these years without the support I received from my friends: Daniil, Michael, Neal, Laura and Rachel. And finally, I thank my family for their constant support and encouragement. 


\section{Contents}

Contents iii



List of Figures . . . . . . . . . . . . . . . . . . . . vii

1 Introduction $\quad 1$

2 Background $\quad 5$

2.1 Insect maneuvering flight $\ldots \ldots \ldots \ldots \ldots \ldots$

2.2 Insect wing and its motion . . . . . . . . . . . . . . . 6

2.3 Unsteady aerodynamics of flapping flight . . . . . . . . . . . . . . . . . . . . . . . . . . . . . . .

2.4 Application of quasi-steady approximations . . . . . . . . . . . . . . . . 14

2.5 Flight dynamics . . . . . . . . . . . . . . . . . . . . 16

2.6 Wing actuation . . . . . . . . . . . . . . . . . . . 19

2.7 Development of flapping Micro Air Vehicles (MAVs) . . . . . . . . . . . . . 22

3 Dynamic coupling in dragonfly free flight 25

3.1 Introduction . . . . . . . . . . . . . . . . . . . . 25



3.3 Experimental setup . . . . . . . . . . . . . . . . . . 27



3.5 Description of the turn takeoff maneuver . . . . . . . . . . . . . . . 28

3.6 Calculation of body moment of inertia . . . . . . . . . . . . . . . . . . 29

3.7 Frames of reference and coordinate systems . . . . . . . . . . . . . . 32

3.8 Equations of motion and effect of coupling term . . . . . . . . . . . . . . 33

3.9 Conceptual model . . . . . . . . . . . . . . . . . . . . . 35

3.10 Turning takeoff maneuver and patterns of change in body orientation . . . . . . . 36

3.11 Effect on angular velocities during the course of maneuver . . . . . . . . . . . 37

3.12 Extra mechanism to enhance maneuverability . . . . . . . . . . . . . . . . 40

3.13 Comparison between two maneuvers of one dragonfly . . . . . . . . . . . . . 42

3.14 Flight direction change with minimum effort . . . . . . . . . . . . . . 43

3.15 Acknowledgement . . . . . . . . . . . . . . . . . 46

4 Cicada flight mechanics $\quad 47$

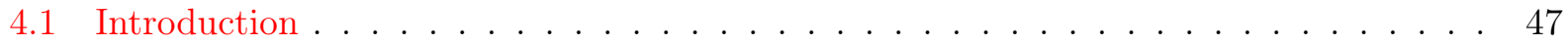

4.2 Tracking and measurement of cicada motion in free flight . . . . . . . . . . . . 49

4.3 Quasi-steady calculation of aerodynamic force . . . . . . . . . . . . . . . . 50

4.4 Body kinematics of cicada free flight . . . . . . . . . . . . . . . . . 50

4.5 Wing kinematics . . . . . . . . . . . . . . . . . 52 
4.6 Investigation of the flexibility of aerodynamic force direction . . . . . . . . . . 54

4.7 Control of the force direction and magnitude . . . . . . . . . . . . . . 59

4.8 Change of flight path . . . . . . . . . . . . . . . . . . 62

4.9 Discussion . . . . . . . . . . . . . . . . . . . . . . . . 64

4.10 Conclusions . . . . . . . . . . . . . . . . . . 66

4.11 Acknowledgement . . . . . . . . . . . . . . . . . 66

5 Damselfly yaw turn $\quad \mathbf{6 7}$

5.1 Introduction . . . . . . . . . . . . . . . . . 67

5.2 Damselflies; Body and wing morphology . . . . . . . . . . . . . . . 69

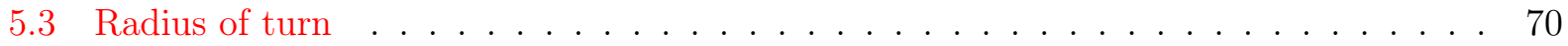

5.4 Wing area calculation . . . . . . . . . . . . . . . . 71

5.5 Quasi-steady estimation of the aerodynamic yaw torque . . . . . . . . . . . 71

5.6 Body kinematics in yaw turn . . . . . . . . . . . . . . . . . . . 74

5.7 Wing kinematics . . . . . . . . . . . . . . . . . . . . 76

5.8 Aerodynamic yaw torque generation . . . . . . . . . . . . . 84

5.9 Discussion . . . . . . . . . . . . . . . . . . . . . . . 86

5.10 Acknowledgement . . . . . . . . . . . . . . . . . . . . 89

6 Integrating wing into the dynamics of flapping flight $\quad 90$

6.1 Introduction . . . . . . . . . . . . . . . . . . . . . . 90

6.2 Wing morphology and kinematics . . . . . . . . . . . . . . . . . 92

6.3 Dynamics of the wing pitching . . . . . . . . . . . . . . . . . 94

6.4 Aerodynamic pitching torque model . . . . . . . . . . . . . . . 96

6.4 Added mass . . . . . . . . . . . . . . . . . . . . . . . . . 98

6.5 Validation of the prediction results with experiment . . . . . . . . . . . 99

6.6 Numerical simulation of the wing-air interactions . . . . . . . . . . . . . 100

6.7 Non-dimensional numbers governing the dynamics of the wing pitch . . . . . . . 102

6.8 Passive wing pitching . . . . . . . . . . . . . . . . . . . 104

6.9 Effect of the body rotations on the wing pitch . . . . . . . . . . 107

6.10 Conclusions . . . . . . . . . . . . . . . . . . . . . . . . 112

7 Passive wing Pitch Asymmetry (PPA) in aerial turns of free flying insects 115

7.1 Introduction . . . . . . . . . . . . . . . . . . . 115

7.2 Damselfly's yaw turn dynamics . . . . . . . . . . . . . . . . . 117

7.3 Response of the physics-based model of the wing to the body rotation . . . . . . . 122

7.4 Estimation of the damselfly Wing hinge stiffness . . . . . . . . . . . . . 128

7.5 PPA in damselfly yaw turn . . . . . . . . . . . . . . . . . . 128

7.6 Force and torque due to the PPA . . . . . . . . . . . . . 130

7.7 Broader application of the wing-body coupling in insect flight . . . . . . . . . 134

7.8 Energy saving due to PPA . . . . . . . . . . . . . . . . . 136

7.9 Conclusions . . . . . . . . . . . . . . . . . . . . 139

8 Conclusions $\quad 141$

8.1 Summary of contributions . . . . . . . . . . . . . . . . . . . 141

8.2 Suggestions for future work . . . . . . . . . . . . . . . . . 144

$\begin{array}{ll}\text { Bibliography } & 146\end{array}$ 


\section{List of Tables}

3.1 Morphological data base of dragonflies in experiment. . . . . . . . . . . . . . . . . . 27

3.2 Geometric Data of each slice of the dragonfly body . . . . . . . . . . . . . . 31

3.3 DCA comparison in two different maneuvers of the same dragonfly. . . . . . . . . . 43

4.1 Wing loading of several species of flying animals. . . . . . . . . . . . . 48

4.2 Performance parameters of different cicada flights . . . . . . . . . . . . . 52

6.1 Cauchy number of several species of insects. . . . . . . . . . . . . . 105

6.2 Slope of the variation of wing pitch and force coefficient with normalized body yaw velocity.110

6.3 Slope of the variation of wing pitch and force coefficient with normalized body roll velocity.112

7.1 Morphology of damselflies. . . . . . . . . . . . . . . . . . . 116

7.2 Kinematics of the body yaw maneuver . . . . . . . . . . . . . . . . . 121

7.3 Measured and predicted yaw velocity half-life for three damselfly species . . . . . . 134 


\section{List of Figures}

2.1 Wing tip trajectory asymmetry due to body motion in aerial maneuver of cicada. . . 9

2.2 Body deflection in aerial maneuver of dragonfly. . . . . . . . . . . . . . . 17

2.3 Flapping Micro Air Vehicles. . . . . . . . . . . . . . . . . . . . . . 23

3.1 Dragonfly turning takeoff. . . . . . . . . . . . . . . . . . . . . 29

3.2 Dragonfly model for MOI calculations. . . . . . . . . . . . . . . . 30

3.3 DCA conceptual model. . . . . . . . . . . . . . . . . . 36

3.4 Body kinematics of dragonfly turning takeoff maneuver. . . . . . . . . . . . . . 38

3.5 DCA in dragonfly free flight. . . . . . . . . . . . . . . . . . . 39

3.6 Effect of DCA on dragonfly body reorientation. . . . . . . . . . . . . . . . . . . . . . . . . . .



3.8 Optimized flight reorientation. . . . . . . . . . . . . . . 45

4.1 Cicada in free flight. . . . . . . . . . . . . . . . . . . . . 49

4.2 Validation of quasi-steady force calculations in cicada. . . . . . . . . . . . . 51

4.3 Reconstructed wing and body of cicada in free flight. . . . . . . . . . . . . . . 53

4.4 Wing tip trajectory in cicada free flight. . . . . . . . . . . . . . . 54

4.5 Wing and body kinematics of cicada in Flight 1. . . . . . . . . . . . . . . . . 55

4.6 Wing and body kinematics of cicada in Flight 2. . . . . . . . . . . . 56

4.7 Wing and body kinematics of cicada in Flight $3 . \ldots \ldots \ldots$

4.8 Wing and body kinematics of cicada in Flight $4 \ldots \ldots \ldots$. . . . . . . . 58

4.9 Aerodynamic force direction relative to the body in cicada cruise. . . . . . . . . . . 60

4.10 Orientation of the aerodynamic force relative to the body normal in cicada free flight. 61

4.11 Effect of wing trajectory on aerodynamic pitch torque in cicada flight. . . . . . . . 63

5.1 Three species of damselfly. . . . . . . . . . . . . . . . . . . 68

5.2 Morphology of three damselfly species. . . . . . . . . . . . . . . . . 70

5.3 Damselfly wing model used for area measurements. . . . . . . . . . . . . . . . . . 72

5.4 Definition of the wing and body coordinate systems. . . . . . . . . . . . 73

5.5 Damselfly body motion during an aerial yaw turn. . . . . . . . . . . . . 75

5.6 Wing kinematics of damselflies $\# 1$ in yaw turn. . . . . . . . . . . . . . . 78

5.7 Wing kinematics of damselflies $\# 2$ in yaw turn. . . . . . . . . . . . . . . . . 79

5.8 Wing kinematics of damselflies $\# 3$ in yaw turn. . . . . . . . . . . . 80

5.9 Wing tip trajectory in the body fixed coordinate system for damselfly $\# 2$. . . . . . 81

5.10 Correlation between the kinematics of bilateral wings. . . . . . . . . . . . 83

5.11 Aerodynamic yaw torque. . . . . . . . . . . . . . . . . . . 84

5.12 Mechanism of torque generation in damselfly yaw turns. . . . . . . . . . . 87 
6.1 Conventional model of the flapping flight dynamics. . . . . . . . . . . . . . . . 91

6.2 Proposed model of the flapping flight dynamics. . . . . . . . . . . . . . . . . 92

6.3 Insect wing model. . . . . . . . . . . . . . . . . . . . . . . . . 93

6.4 Validation of the wing pitch angle prediction. . . . . . . . . . . . 101

6.5 Dynamics of passive wing pitch across different insect wing kinematics and morphology.106

6.6 Variation of force coefficient with $C h$ for passively pitching wing. . . . . . . . . 106

6.7 Passive bilateral wing pitch difference versus $\Omega_{y a w} / \omega \ldots \ldots$. . . . . . . . . . 109

6.8 Bilateral force coefficient difference versus $\Omega_{y a w} / \omega \ldots \ldots \ldots$. . . . . . . . . . . 111

6.9 Passive bilateral wing pitch difference versus $\Omega_{\text {roll }} / \omega \ldots \ldots$. . . . . . . . . . . 112

6.10 Bilateral force coefficient difference versus $\Omega_{\text {roll }} / \omega \ldots \ldots$. . . . . . . . . . 113

7.1 Motion reconstruction of the 160 degree turn of damselfly $\# 1 . \quad \ldots \ldots$. . . . . 118

7.2 Wing and body kinematics of damselfly $\# 1 . \ldots \ldots$. . . . . . . . . . . 119

7.3 Stroke plane in damselfly yaw turns . . . . . . . . . . . . . . . . . . 120

7.4 Ratio of the average body to wing angular velocity in damselfly yaw turn. . . . . . 120

7.5 Average pitch angle versus the corrected wing angular velocity. . . . . . . . . . . 123

7.6 Coordinate systems of the wing and the body. . . . . . . . . . . . . . . . 124

7.7 Wing and body velocity in Damselfly in yaw turn. . . . . . . . . . . . 126

7.8 Passive wing pitch asymmetry during a model turn maneuver. . . . . . . . . . . . 127

7.9 Estimation of the damselfly wing hinge stiffness. . . . . . . . . . . . . . . . . 129

7.10 Prediction of the wing pitch in damselfly yaw turn . . . . . . . . . . . . . 130

7.11 Horizontal force coefficient asymmetry. . . . . . . . . . . . . . . . . 131

7.12 Measured and predicted yaw velocity. . . . . . . . . . . . . . . 134

7.13 Predicted versus measured wing pitch angle in insect maneuvers. . . . . . . . . 135

7.14 Kinetic energy decay with and without wing-body coupling in yaw turns. . . . . . . 137 


\section{Chapter 1}

\section{Introduction}

It has been over a century since the first controlled flight of manmade machines. This unprecedented feat enabled humans to conquer the skies with aircrafts that employ powerful engines and large lifting surfaces. However, while there has been much development in understanding fixed winged flight, not much known is known about flapping flight which has evolved in nature for hundreds of million of years, allowing flying insects to surpass other animals in locomotion. Unlike manmade aircraft, insects fly at miniature scales at which current technologies cannot compare in performance. To gain understanding of how insects fly, scientists and engineers have pursued this topic for decades. Much progress has and is being made toward understanding the unsteady aerodynamics of flapping flight. Yet many aspects of how insects maneuver and control their flight is poorly understood.

In this thesis, our overarching goal is to investigate some of the fundamental questions about the flapping flight dynamics such as; what are the similarities and differences between the fixed versus flapping wing flight dynamics, or how the frequency of the wing flapping affects the overall performance of flight. To address our goal there are two main challenges to overcome. First, most observations and direct measurements have been conducted on high flapping frequency insects such as fruit flies. With the lack of quantitative information on the detailed wing and body kinematics as well as flight performance, it is difficult to evaluate how the dynamics of the insect flight is affected by the flapping frequency. Second, the majority of the methods and techniques that are currently employed in the study of the insect flight are those that were borrowed from fixed wing flight dynamics. This is because at high flapping frequencies, motion of the wings is so fast compared to that of the body that body only endures the effect of the stroke averaged aerodynamic force and torque. 
Therefore, the time dependency of the system dynamics can be eliminated and the traditional techniques used in the fixed wing flight can be applied [Cheng and Deng, 2011]. However, the validity of these assumptions in low frequency flapping flight is questionable. Without appropriate theoretical modeling and experimental validations, it is not possible to gain a comprehensive understanding of the insect flight, across the wide range of their morphology and kinematics. These challenges are addressed in this thesis through two main areas of research:

(1) Studying free maneuvering flight of several species of low flapping frequency insects and identifying the key maneuverability mechanisms. These studies focused on different areas such as the change in the body motion, the connection between the wing and the body motion and the generation of aerodynamic torque for the body rotations. Lessons learned from these studies not only improves our understanding of the flight mechanics of the specific insect species, but also provides guidelines for design of agile flapping wing Micro Air Vehicles (MAVs).

(2) The second branch of the work establishes an interface between the wing and the body motion by integrating bio-mechanical models of the wing with appropriate models of the unsteady aerodynamics of flapping flight. We used this model to investigate the basics of the wing kinematics control and force alternation in maneuvering flight. Lastly, we combined the wing and the body dynamics to achieve a comprehensive view on the dynamics of flapping flight.

The pursuit of these goals is documented in this thesis, with one chapter focusing on the overview on the insect flight related topics that were investigated in this dissertation. This chapter is followed by five chapters that include the major results of this work. Each result chapter stands alone, starting with an introduction and ending with the conclusions. The final chapter of the dissertation summarizes the major contributions of the dissertation and list some possible avenues to continue this research along. The layout of the thesis is outlined in more detail as follows.

Chapter 2 gives an overview on the insect flight capabilities, reviews the unsteady aerodynamics of flapping flight and discusses the applicability of quasi-steady approximations of the force generation mechanisms. The chapter is continued by an overview of the flight dynamics of insects and current methodologies and approaches employed in this field, review of the physiology and physics of the wing actuation and finally it outlines the state of the art in development of flapping Micro Air Vehicles (MAV). 
Chapter 3 is an investigation of a specific type of aerial maneuvers of dragonflies that is termed as turning takeoff within which the insect changes the flight heading while the center of mass is being elevated. While the previous studies on the flapping wing flight dynamics mostly focused on the separated longitudinal and lateral dynamics, the contribution of the current work is to show that the coupling of angular velocities about different principal axes may have significant effect on the flight dynamics. We show that combining rotations about pitch and roll axes of the body allows a dragonfly to perform fast flight heading changes. More importantly, our results indicate that by controlling the roll velocity, dragonflies can execute a wide variety of flight heading changes with minimal alternation in the average aerodynamic pitch and yaw torque.

Chapter 4 investigates the underlying mechanics of cicada free flight through studying a wide variety of flight modes, including a forward flight, a banked turn, an Immelmann turn and a vertical takeoff. Using the high-speed videography and a 3D surface reconstruction technique, we discovered that the motion of the cicada wings and associated aerodynamic force directions present a stereotypical pattern regardless of the body orientation, indicating a common and simple control strategy employed in these flights. The results of this research opens several new avenues of research in the study of the insect flight dynamics, neuromuscular control of the wing and body motion, energy economy in maneuvering flight, and control of the wing kinematics and flight forces in insect flight.

Chapter 5 presents experimental measurements on the wing and body kinematics of three different species of damselfly in free aerial yaw turns. Providing these measurements is one of the main contributions of this work, considering the scarce number of reports on the flight of damselflies. We also systematically analyzed the flight data to identify the key differences and similarities in techniques employed by different species of damselfly to execute a yaw turn. Furthermore, we compared the yaw turns of damselflies to those of higher flapping frequency insects, such as flies, with regard to the wing and body kinematics as well as the underlying mechanisms. Our results indicate the manipulation of the angle of attack of the bilateral wings is the single most important parameter in generating the yaw torque to execute the maneuver.

Chapter 6 represents a model of the flapping wing system which mimics the functional properties of the wing and the hinge structure, combined with a model of the aerodynamic force and torque. The dynamics of the wing is systematically investigated and characterized based on the wing geometry, kinematics and its structural properties across a wide range relevant to the flying 
insects. The results of the parametric study can be used to inform design of insect-size flapping wings. Furthermore, we showed a mechanism by which the motion of the body can alter that of the wing. The wing response to lateral body rotations is systematically investigated as well.

Chapter 7 investigates the connection between the wing and the body dynamics via analyzing fast yaw turns of three different species of damselflies. In this combined experimental and theoretical study, we show that the dynamics of the wings and the body are coupled together in low frequency flapping flight. As a results, damselflies benefit from a passive mechanism termed as Passive wing Pitch Asymmetry (PPA) to maintain the body rotational velocity. The model was has also successfully predicted the wing pitch angle of a dragonfly in turning takeoff and a cicada in a banked-turn, showing its broader application to the maneuvering flight of different insect species. The model also provides a framework for linking morphology, kinematics and dynamics of the wing and the body of the flying insects.

Chapter 8 summarizes the conclusions from the five results chapters and lists the major contributions of the thesis. This is followed by a brief discussion of the limitations of the methods and conclusions. At the end, some suggestions were made for the future continuation of the work. 


\section{Chapter 2}

\section{Background}

Evolution of flight in nature has been central to diversity of flying animals [Dudley, 2002]. The capacity to move in $3 \mathrm{D}$ space provided them with superior abilities in predator-pray interactions and in exploring novel habitats. This was frequently argued to cause exceptional taxonomic richness of the winged insects [Dudley, 2002]. Conservative estimations indicate that there are between 5 to 10 million different species of insects [Gaston, 1996] (whereas there are only 42,000 species of vertebrate). The richness of the insect species also lead to a diverse range of flight capabilities. Some insects are capable of fast flights, precise hovering and executing three dimensional changes in the flight trajectory within portion of a second, while others may exhibit irregular and erratic flights or even be incapable of making a controlled landing [Dudley, 2002]. Insects are also remarkably diverse in shape, size, body and wing kinematics. Some of them, such as butterflies, flap their wings only 4-5 times a second, whereas others, such as minute ceratopogonidae flies oscillate their wings at frequencies as high as $1000 \mathrm{~Hz}$. Although flying insects were a subject of interest to scientists for centuries, the functional impact of these differences with regard to the aerial locomotion is not yet understood [Dudley, 2002].

Insects fly in flow regimes that are not accessible to human-made flying vehicles. They are miniature in size and extremely agile which makes them even more attractive to scientists. The superiority of insect flight is a result of many elements including unsteady aerodynamics of the flight, neuro-muscular actuation and control of the wing, rapid sensory systems and body dynamics. Scientists studied insects for decades to unreveal different aspects of their flight. Flies have specially served as a model for many features of flight mechanics [Muijres et al., 2014, Cheng et al., 2010], 
unsteady aerodynamics [Fry et al., 2003], wing kinematics control [Lehmann and Dickinson, 1998], sensory system [Muijres et al., 2014] and muscle physiology [Lehmann and Dickinson, 1997], in study of insect flight.

\subsection{Insect maneuvering flight}

Linear flight alone is insufficient to ensure aerial locomotion and survival [Dudley, 2002]. Most importantly, insects should be able to ascend, descend and change the flight trajectory. The capacity to change the flight trajectory is specially important in predator-prey interactions. Aerial maneuvers of different insect species were studied widely in the last decades and many aspects of maneuverability and stability in insect flight were explored. Although insects are capable of performing a variety of aerial maneuvers, technical challenges in acquiring accurate three dimensional data limited diversity and depth of our knowledge about flapping flight in nature. Most of the early investigations of insect flight were conducted on tethered insects. Recently high speed photogrammetry technology together with different auto-tracking and reconstruction techniques [Ristroph et al., 2009, Koehler et al., 2012, Wagner, 1986] inspired more comprehensive and accurate studies on the different aspects of the insect flight. Aerial maneuvers of different insects have been recorded and detailed body and wing kinematics were measured. In free flight, predation and evasive flights have been studied, as well, which revealed significant information about population dynamics and individual fitness [Combes et al., 2012, McLachlan et al., 2003].

\subsection{Insect wing and its motion}

Insect wings are thin cuticular structures enforced by veins that spread across the wing in complex patterns. Leading edge of the wing contains thickened veins that provide structural rigidity. There are several radially stretched flexion lines on the wing which represent regions of increased flexibility and along them wing can deform and yield variable camber [Dudley, 2002]. Wing mass, that mostly arises from the wing venation, varies among species with wing to body mass ratio ranging between 0.5-4 \% in dipterans and hymenopterans [Ellington, 1984b] and 3-10\% [Betts and Wootton, 1988] in butterflies. The distribution of the wing mass has mechanical importance. Spanwise mass distribution defines the wing's moment of inertia about its hinge point and therefore indicates 
the power required for flapping the wings. The center of mass of the insect wings usually lies at about 30 to $40 \%$ of the wing length from the wing hinge, reducing the wing moment of inertia [Ellington, 1984a]. The chordwise distribution of the wing mass also is important in easing wing rotation at stroke reversal. The wing center of mass is located below the longitudinal axis of rotation of the wing [Ennos, 1988]. Therefore, the inertial forces due to the wing acceleration helps flipping the wing at the stroke reversal [Ennos, 1988, Bergou et al., 2007].

Geometrical wing shape is also of great importance in generation of the aerodynamic force. Wing total area directly affects the magnitude of the aerodynamic force. The wing loading, defined as the ratio of the body mass over the wing area, is an indicative of the flight performance. Wing area tends to increase linearly with the body dimension, whereas body mass is a function of volume increasing with the cube of the body dimension. Therefore, insects with larger bodies usually have higher wing loading. In addition to the area, wing's aspect ratio, defined as the ratio of the wing span squared to the wing area, is used to described the wing shape. Aspect ratio of the insect wings varies in a wide range from 2, for some butterflies, to 10, for some Odonata [Dudley, 2002]. Additionally, virtual mass of the wing is derived from the wing geometry. It is proportional to the chord length of the wing and the air density [Ellington, 1984a]. Therefore, the spanwise distribution of the wing chord influences this variable. Virtual mass of the wing which represents the volume of the air accelerating with the wing, can be comparable to the wing mass itself and therefore can play an important role in the required power for flapping the wing [Dudley, 2002].

In addition to generating aerodynamic lift to stay aloft, wings of the insect generate the propulsive force for locomotion and modulate the force magnitude to execute aerial maneuvers. Motion of the wings directly influences the magnitude as well as the temporal variations in the aerodynamic force. While the details of the wing kinematics varies among species of insects as well as from one flight mode to another, the general motion is composed of two translational phases, downstroke and upstroke, and two rotational phases, supination and pronation. Wing rotation occurs at the transition between the half strokes and flips the leading edge of the wing allowing generation of lift in both half strokes. Wings of most insects flap back and forth in a plane, referred to as stroke plane. The orientation of the stroke plane relative to the body longitudinal axis is anatomically limited in most insects [Dudley, 2002], varying in small range. The mean number of oscillatory cycles of the wing per second, in the stroke plane, is defined as the flapping frequency, ranging between 10-1000 
among different insects. Flapping frequency is the most important factor determining the velocity of the wing relative to the air, which is directly associated with the aerodynamic force generation. Within an approximately unvarying behavioral context, flapping frequency of individual insects remain unchanged [Dudley, 2002]. Flapping amplitude is measured based on the range of the motion of the wing in the stroke plane. While insects such as fruit flies flap their wings in large amplitudes (120-160 deg) [Fry et al., 2003], others such as dragonflies have significantly smaller flapping amplitudes, sometimes as low as 40-50 deg. Deviation of the wing from the stroke plane causes oval or figure eight wing tip trajectories. deviation of the wing from the stroke plane is under active control for some insects such as flies [Tu and Dickinson, 1996], whereas others insects such as bumblebee [Dudley and Ellington, 1990] and moth [Willmott and Ellington, 1997] show systematic variations in the wing tip trajectory with forward speed. The geometric angle of attack of the wing, the orientation of the wing chord relative to the stroke plane, is modulated by pitching the wing about an axis which is close to the leading edge. Together with the wing velocity, the wing pitch angle has a large influence on the aerodynamic force generation. studies on free flight maneuvers of inscets shows that they primarily modulate wing pitch to generate in flight turns [Ellington, 1999, Bergou et al., 2010]. 




Figure 2.1: Wing tip trajectory asymmetry due to body motion in aerial maneuver of cicada. Wing tip trajectories of a cicada in a banked turn are shown with two different colors. Motion of the body stretches the trajectory of the outer wing and compresses that of the inner wing.

Insects modulate their wing kinematics to change the aerodynamic force magnitude and direction. The body motion also affects the net motion of the wing relative to the air and therefore influences the aerodynamics of flight. In maneuvering flights, for instance, the rotation of the body can cause significant asymmetry in the trajectory of the bilateral wings. This effect is more pronounced in low flapping frequency insects where the rotation of the body within one wingbeat is large. Figure 2.1 shows the bilateral wing tip trajectory of a cicada in a banked turn. It is evident that the motion of the body can potentially alter the wing trajectories and therefore affect the net wing kinematics. 


\subsection{Unsteady aerodynamics of flapping flight}

Study of aerial locomotion is strongly tied to the aerodynamic forces generation. The flapping wings transfer momentum to the surrounding air and the subsequent reaction forces on the wings support the body weight and propel the insect in the air. Air density, representing inertial resistance of the fluid to motion, and viscosity, representing internal resistance to deformation, are the essential determinants of aerodynamic force generation. The ratio of inertial to viscus forces is defined as Reynolds number (2.1).

$$
R e=\rho L U / \mu
$$

where $L$ and $U$ are the characteristic length and velocity, respectively. Due to the small length and slow velocities, Re number of insect flight is small (ranging between 100-10000), indicating that viscous effects may play an important role in force generation. Both lift and drag generations are a function of $R e$. Unsteadiness of the flow and force generation is defined via another non-dimensional number named Strouhal number, St, also referred to as reduced frequency $((2.2))$.

$$
S t=f c / U
$$

where $f$ is the flapping frequency. Force generation in flapping flight is dominated by unsteadiness of the motion and the flow. Larger $S t$ numbers indicate larger unsteadiness of the flow. By examining the flapping frequency and amplitude of more than 40 species of bats, birds and insects in cruising flight, Taylor et. al. [Taylor et al., 2003] showed that these flying animals operate within a narrow range of $S t$ numbers; between 0.2 and 0.4. It was argued that this range provides the largest propulsive efficiency, defined as the ratio of thrust to power coefficients, by generating optimal wake formation. However, other studies show that the swimming economy (defined as the ratio of freeswimming velocity to the input power) varies monotonically with swimming velocity, implying that swimming slower will allow larger distance traveled per unit energy consumption [Kem, ].

The importance of unsteadiness in aerodynamics of flapping flight is largely known. In fact, it has been argued that conventional aerodynamic mechanisms in steady flight cannot explain the generation of lift by the wings of small insects [Ellington, 1984b]. Several unsteady mechanisms are

known to play a vital role in force generation of insect wings. These mechanisms are tightly asso- 
ciated with the complex and unsteady motion of the wing through the air [Dickinson et al., 1999]. The first unsteady effect to be identified was named "clap-and-fling", cause by the close proximation of the wings preceding pronation which increases development of circulation in downstroke [Spedding and Maxworthy, 1986]. During clap-and-fling, that was first described by Weis-Fogh for wasp, the wings start from clap position at the end of upstroke. Each one of the wings then start to rotate about its own trailing edge and air flows into the region between the wings. The net angular momentum of the air remains zero since the rotation of the wings are in opposite directions. Circulation begins to develop about each wing and continues as the wings start moving in downstroke. When the wings separate completely circulation is substantial about each wing although there has been no vortex shedding [Dudley, 2002]. However, this mechanism is not used by all insects [Marden, 1987] and thus cannot represent a general approach for enhancing lift production.

Another potential unsteady aerodynamic mechanism is the added (or virtual) mass effect, associated with the fluid mass that moves with the wing as wing accelerates in the air. The magnitude of added mass forces, which varies with wing geometry and its orientation relative to the flapping axis [Jones, 1941, Scruton et al., 1941], can be comparable to the wing mass [Ellington, 1999]. Although the instantaneous magnitude of the added mass force is high, the contribution to the stroke-averaged force may not be significant due to relatively symmetric wing kinematics [Dudley, 2002]. However, added mass effect was shown to play an important role in generating torque about the wing pitching axis, helping the wing to flip at stroke reversal [Bergou et al., 2007, Ishihara et al., 2009, Whitney and Wood, 2010]. The importance of the added mass in maneuver is also unclear but may be significant due to more complex wing kinematics.

Stabilized leading edge vortex is one of the well known unsteady mechanisms identified in insect flight, also known as "delayed stall" or "dynamic stall". Flapping at large angles of attack, a strong vortex is generated on the wing leading edge. As the angle of attack increase, the vortex enlarges which can potentially result in burst and loss of aerodynamic force. It has been argued that in rotary and flapping wings, the radial pressure difference due to variable flow velocity along the span convects the vortex to the wing tip, preventing it from growing too large. This stabilizes the leading edge vortex and delays stall. Therefore, the insect wing can maintain high lift generation that occurs right before stall [Ellington et al., 1996, Van Den Berg and Ellington, 1997, Willmott et al., 1997]. Flow visualizations on tethered insects has shown that in contrast to downstroke, no stable leading edge vor- 
tex is evident in upstroke which results in less aerodynamic force magnitudes [Willmott et al., 1997]. The mechanism of the leading edge vortex attachment may be different at low Re numbers such as that of smaller insects like fruit flies. In an experimental investigations, Birch et. al. placed vertical plates along the wing span to limit spanwise flow at Re 200. They found that the structure of the leading edge vortex is not affected by the attenuated spanwise flow through the vortex core. They hypothesized that at low Re numbers the downward flow induced by both the tip vortex and the previously shed vortex, decreases the effective angle of attack and delays stall [Birch and Dickinson, 2001]. Finally, in a combined experimental and theoretical approach, Lentink and Dickinson [Lentink and Dickinson, 2009] argued that generation of the leading edge vortex is independent of the $R e$ number (for $100<R e<14000$ ) and is caused by "quasi-steady" centripetal and Coliriolis accelerations that result from propeller-like swiping of the wing through the air. They concluded that the back and forth motion of a flapping wing does not play a significant roll in this regard. To further study correlation between the forces and leading edge vortex structure Birch et. al. [Birch et al., 2004] estimated force from the velocity filed in an enclosed area around the wing, by using a method introduced in [Noca et al., 1997]. Equation 2.3 gives steady force on each section of the wing along the span.

$$
F=\rho \int_{A} u \times \omega d A-\rho \oint_{S} n . u(x \times \omega) d s+\oint_{S} n .[x \cdot(\nabla \cdot T) I-x(\nabla . T)+T] d s
$$

Where $A$ is the area enclosing the wing section, $S$ is the boundary of this area, $u$ is velocity, $\omega$ is vorticity, $n$ is the outward unit normal vector, $x$ is position vector and $T$ is the stress tensor which for an incompressible flow is given by equation 2.4 .

$$
T=\mu\left(\nabla u+\nabla u^{T}\right)
$$

As is expected by continuity equation, change in circulation along the span $(d \Gamma / d z)$ was the same for chordwise and spanwise circulation which means that tip and leading edge vortex can be represented by continues array of vortex filaments bent into the wake. Their study revealed that most of the flapping wing's force (about 70\%) comes from the spanwise circulation, although 2 dimensional steady flow was assumed. They have shown that stability of the leading edge vortex 
won't be affected by change in Re number (from 120 to 1400) nor by inhibiting spanwise flow. Regardless of the stabilizing mechanism, delayed stall, which is a translational mechanism, is the predominant unsteady aerodynamic mechanism. for force generation in large insects [Dudley, 2002].

Rotational circulation is another unsteady aerodynamic mechanism employed by insects. This mechanism is akin to the Magnus effect, which makes a spinning baseball curve from its path. As the baseball spins, it increases the total air velocity on one side and decreases it on the other. It was shown that the wing rotation at stroke reversal has a similar effect and can cause large peaks in force depending on the rotation timing of the wing relative to the reversal of the translational motion [Dickinson et al., 1999]. Rotational circulation is a quasi-steady effect and its magnitude varies with the position of the axis of rotation as well as the magnitude of the wing rotational velocity [Sane and Dickinson, 2002]. Studies on maneuvering flights of insects have shown that they adjust the timing of the wing rotation to modulate the magnitude and direction of the aerodynamic force [Dickinson et al., 1993].

In flapping flight, the back and forth motion of the wing allows the wing to move through its own shed wake causing change in the effective angle of attack. This mechanism is known as wake capture and was investigated by experiments on robotic flapping wings. Dickinson and his colleges showed that a flapping wing continues to generate aerodynamic force at the end of each half-stroke even if it comes to complete stop [Dickinson et al., 1993]. In another study, Birch and Dickinson attempted to isolate the effect of the wake capture from other aerodynamic mechanisms by subtracting forces and flow fields in the first wing stroke from that of the forth stroke [Birch and Dickinson, 2003]. They showed that the wake capture influences force generation via two mechanism; generation of the rotational starting vortex which enhances force generation and attenuation of the effective angle of attack which decreases the force magnitude.

In addition to what we have already discussed, there are other unsteady aerodynamic mechanisms such as wing-wing interaction in four winged insects. In dragonflies, the wake shed from the forewings affects the aerodynamic performance of the hindwings enhancing force production [Wang and Russell, 2007] and reducing power consumption [Lehmann, 2009]. In addition, in maneuvering flights, the wake generated by the hindwings can affect the aerodynamic performance of the forewings [Dong and Liang, 2010]. Another unsteady mechanism reported in insect flight is the wing-body interaction which is adopted by wide body insects such as cicada [Wan et al., 2015]. 
Through a computational investigation, Wan et. al. [Wan et al., 2015] showed that the merging of the thorax vortex with the leading edge vortex enhances lift production.

In addition to rotating about the hinge, wings of the insects flex and deform while flapping. Wing deformations in the insects is mainly passive, resulted from the interaction of the wing structure with the aerodynamic and inertial loads [Combes and Daniel, 2003]. They influence the instantaneous shape of the wing which in return affects the aerodynamic performance. However, the aerodynamic effect of the wing flexibility is not quite known. Recent studies have suggested that wing deformation can improve wake capture [Vanella et al., 2009] or improve lift to drag ratio [Zhao et al., 2009]. However, these effects are minimal and the gross motion of the wing is responsible for the largest portion of the aerodynamic performance.

\subsection{Application of quasi-steady approximations}

An accurate estimation of the aerodynamic force can only be obtained by direct measurement of the forces or employing high fidelity numerical simulations of the flow. Concurrent measurement of the force and the flow field allows investigation of the potential underlying aerodynamic mechanisms of force production. However, these methods are complex and costly, limiting their applicability in large scale data processing and heavy computations such as optimization and flow-body interaction investigations. Therefore, the applicability of the quasi-steady aerodynamics to the force production in flapping flight has been a subject of great interest for decades. The unsteadiness of the flapping flight, which rises from temporal changes in the wing motion, can be interpreted as the superposition of series of consecutive steady state conditions. In this method, the aerodynamic forces are only a function of the current state of the motion, assuming that the flow field do not pose a memory of the previous states. Such estimations were previously applied to insect flight. Osborne [Osborne, 1951] conducted the first detailed quasi-steady approach to insect flight that neglected the induced velocity in estimation of the wing relative velocity. There were a number of more accurate methods developed for estimation of the aerodynamic force in flapping flight. For instance, Ellington [Ellington, 1984b] developed a detailed vortex wake theory to incorporate the effect of the induced flow into the prediction of the wing's effective angle of attack. He used a Rankine-Froude actuation disk model. He modified the otherwise constant downward momentum flux produced by 
Rankine-Froude actuator disk to reflect periodically applied momentum pulses across the area as the wing flaps back and forth within the stroke plane. In addition, he incorporated the effect of spatial variability of the wing circulation across the span. He showed that these modifications improve the estimation of the induced velocity by 15 percent in hovering. More recently, Nabawy and Crowther [Nabawy and Crowther, 2014] employed a method based on the combination of lifting line theory and actuator disk to quantify the losses due to non-uniform downwash, tip losses and finite flapping amplitude on the induced power factor. They obtained good agreements between their prediction of the distribution of the wing circulation along the span with experimental and numerical data in literature. The importance of this sort of analysis is the insight it provides regarding the effect of the wing shape and kinematics on the aerodynamic performance without necessitating intensive parametric studies.

Another class of studies, attempt to incorporate unsteady aerodynamic mechanisms into the force calculations using theoretical modelings combined with numerical and experimental approaches. In these studies the underlying elements of the wing morphology and kinematics that affect the aerodynamic performance are identified through theoretical modeling. The non dimensional coefficients are then quantified using high fidelity approaches. For instance, the augmented force due to dynamic stall can be quantified by dimensionless lift and drag coefficients as a function of angle of attack. This was achieved by employing experimental [Dickinson et al., 1999] and numerical approaches [Wang et al., 2004]. Unsteady ideal flow theory was used to build models of the rotational circulation in flapping flight. Despite the complexity of the 3D motion of the wing and small Re number in insect flight, studies have shown that this model can accurately predict the augmentation in the circulation due to its rotation. Modeling and validations were independently performed in experiment, using dynamically scaled robotic wings [Dickinson et al., 1999, Sane and Dickinson, 2002] and numerical simulations based on falling plates [Pesavento and Wang, 2004]. Experiments show that the coefficient of the rotational circulation varies with the location of the rotation axis, as predicted by unsteady aerodynamics theory. However, it also varied with the magnitude of the wing angular velocity, in oppose to the theoretical predictions. The slope of the variations of the coefficient of rotational circulation with the wing angular velocity is smaller for rotation axes located closer to the wing leading edge, which is the case for insect wings. Effect of the added mass was also implemented treating the wing as a series of 2 dimensional airfoils that do not interact along 
the wing span [Whitney and Wood, 2010]. This method is referred to as blade element method and is commonly used in quasi-steady approximation of the aerodynamic force on three dimensional wings [Dudley, 2002]. Several attempts were conducted to quantify the effect of the wake capture on the force production [Sane and Dickinson, 2002]. However, no theoretical modeling of this effect is available. Yet, the good agreement that was achieved between the prediction of the current theoretical models and the experimental and numerical measurements of the force indicates that this effect plays a secondary role in aerodynamics of flapping flight.

\subsection{Flight dynamics}

While unsteady aerodynamics of flapping flight plays a fundamental role in insect flight, flight would have not been possible without implementing proper means and techniques for adjusting, controlling and stabilizing the motion. Just as Wright brothers were the first to implement control devices into their airplane that made the first stable flight possible. Investigating the dynamics of flight usually involves combining different aspects of flight including rigid body dynamics, aerodynamics, sensory feedback and wing actuation. However, there are many challenges facing these investigations. In flapping flight the nature of the flight forces is time varying (and in simple cases periodic) which makes the motion nonlinear and time dependent. The nonlinearities arise from coupling between the velocities of the body (due to three dimensional motions) as well as the coupling in the aerodynamic terms which are a function of both wing and body motions. The latter effect is even more pronounced in low flapping frequency flight where the time scales of the wings and body motions are close. for larger insects, which usually have lower flapping frequencies, the rigid body assumption may be questionable as well. for instance, dragonflies actively use their tails in turning [Bode Oke et al., 2015]. Figure 3.2 shows a selected sequence of images of a dragonfly in turning takeoff. It is evident from the figure that the tail deflects significantly during the maneuver. Both magnitude and velocities of these deflection are potentially large, indicating that the rigid body assumption may not be valid in the analysis of these flights. My coworker and I studied the potential effect that tail deflection can pose on the dynamics of turning takeoff flights of dragonflies by defining an optimization problem to find a dynamics configuration of the dragonfly body that will minimize the aerodynamic torque required to execute the turn. Our results show that the 
tail movement significantly reduces the average magnitude of the required torque. In addition, our model predicts that larger deflections are more suitable for faster turns which is in agreement with our observations [Bode Oke et al., 2015]. Tail deflection was observed in other large insects such as locust [Camhi, 1970], hawk moth [Hinterwirth and Daniel, 2010] and even small insects like fruit flies [Gotz et al., 1979].

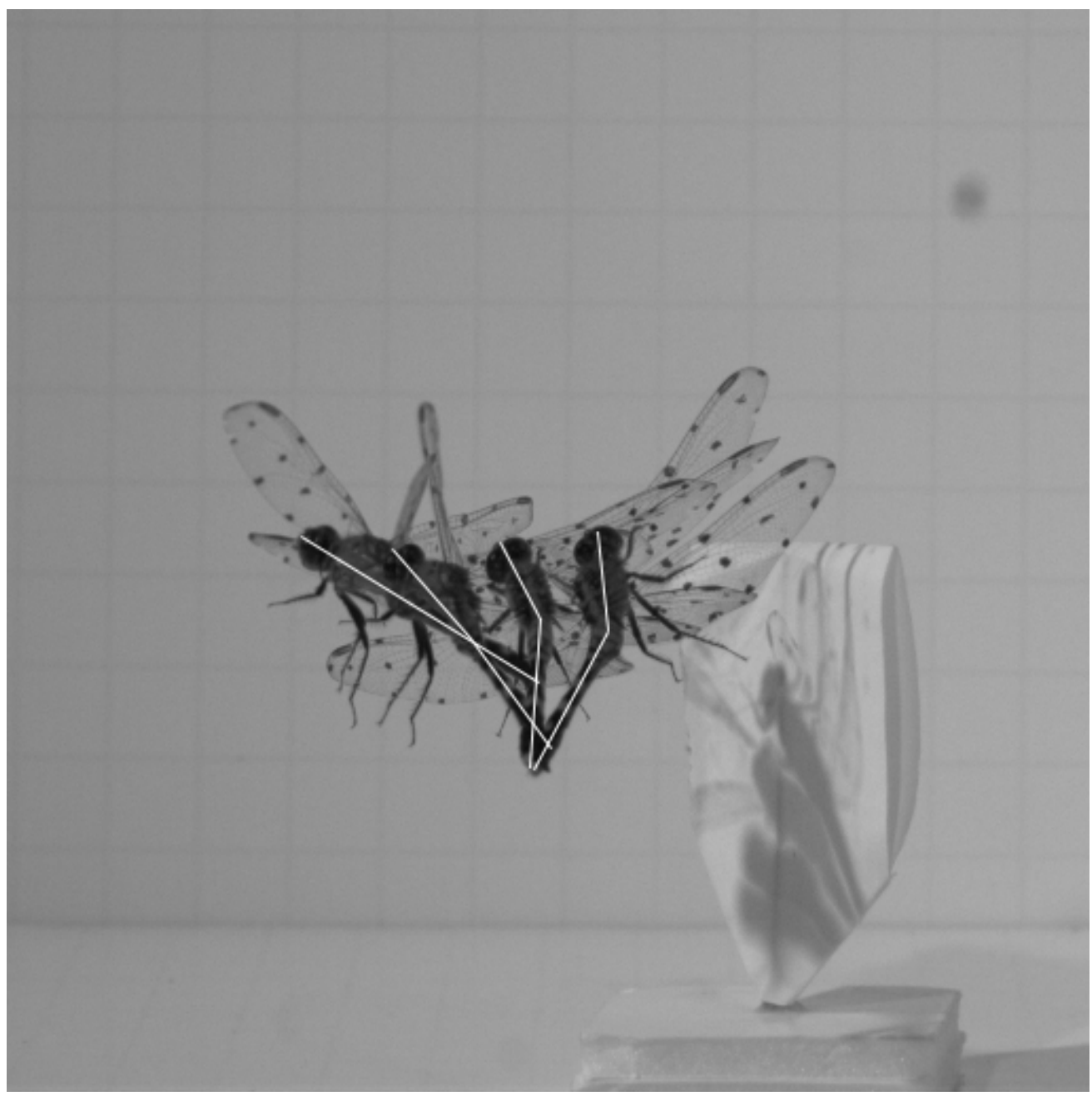

Figure 2.2: Body deflection in aerial maneuver of dragonfly. Tail bending of dragonfly in turning takeoff. The instantaneous magnitude and as well as the velocity of the tail deflections are potentially large in these maneuvers. The while line shows the centerline of the thorax and the tail.

Many techniques employed in the study of insect flight dynamics are borrowed from our knowledge on the conventional aircrafts. For instance, similar to the conventional flight dynamics techniques, stability of the insect flight is commonly investigated by linearizing the equations of motion about a specific flight state (usually equilibrium). These equations can be solved by Floquet theory 
[Nayfeh and Mook, 2008, Wu and Sun, 2012] or by numerical simulations [Taylor and Zbikowski, 2005]. Furthermore, in high flapping frequency flight, another simplification can be applied. Assuming that the time scale of the wing motion is significantly smaller than that of the body motion, the time dependence of the dynamics can be eliminated using averaging theory. In this method only the stroke averaged aerodynamic forces and torques are considered. Therefore, a system of linear equations can be obtained that characterizes the dynamics of the system about a specific equilibrium condition. The sensitivity of the average aerodynamic force and torque to the changes in the flight state are quantified by stability and control derivatives [Taylor and Thomas, 2003]. The behavior of this system can be then identified by analyzing the roots of the characteristic equation. It is important to note that the perturbations may also change the motion of the wing relative to the body, since wings are not rigid attached. In this case, the stability derivatives are different from that of the fixed wing aircrafts. In addition, insects use their wing for modulating the force and torque and those active changes should be included in the form of control derivatives. Stability derivatives are commonly calculated using numerical simulations or quasi-steady approximations based on the fixed wing kinematics in the equilibrium condition [Sun and Xiong, 2005, Zhang and Sun, 2010]. Alternatively Taylor and Thomas [Taylor and Zbikowski, 2005] experimentally measured the stability derivatives of tethered locusts in a wind tunnel. They systematically changed the flight path angle to evaluate the variations in the aerodynamic force and torque. However, in using this method there is no guarantee that the wing kinematics stay invariable by changing the flight condition. In addition,the effect of tethering on the wing kinematics is unknown and therefore the wing kinematics maybe different from that of freely flying insect.

Recent advancements in high speed videography allow researchers to directly measure the response of free flying insects to external disturbances. Ristroph et. al. [Ristroph et al., 2010] investigated the flight autostabilier of fruit flies by artificially inducing aerial stumbles. By attaching a pin to the fly's body they applied torque impulses to freely flying flies and measured their wing and body kinematics before and after the disturbance. To explain the flight recovery behavior they formed a feedback control model that includes the sensory input from the body rotation, the processing of the data and generation of the wing modulations that produces the corrective torque. They discovered that flies process feedback from the body angular velocity and respond to the perturbation via a combined active and passive mechanisms. Similar approach was taken to unreveal 
the control strategy of pitching maneuvers in hawkmoth [Cheng et al., 2011]. The maneuver was trigged by sudden visual stimulus in front of the freely flying hawkmoths and the kinematics of the wings and the body were measured simultaneously. The open loop dynamics of the motion was then extracted by recreating the wing motion on a dynamically scaled robotic wing. Assuming that hawkmoth uses feedback on translational velocity, pitch angle and pitching velocity, they formed a closed loop model of this flight. They show that the control of the body pitching is based on the feedback from both the orientation and angular velocity of the body while the translation is mainly dampened by passive mechanisms. Similarly, pure analytical and numerical approaches were applied to insect flight to understand the architecture of the control system. Cheng and Deng showed that stable body rotations can be achieved by a simple proportional feedback control. They modeled the dynamics of the body using Euler equations governing 6DOF motion of a rigid object whereas the stability and control derivatives were derived using a quasi-steady approximation of the force and torque [Cheng and Deng, 2010].

\subsection{Wing actuation}

In order to understand the insect flight and construct satisfying models which can be used for agile flapping wing Micro Air Vehicle (MAV) design, it is necessary to understand how the complex motion of the wings generates aerodynamic forces and even more importantly, how the force can be altered during the flight. The modulations in the wing kinematics is the primary means by which insects and birds control their flight force. Insects, such as flies, steer and maneuver primarily by altering the wing stroke amplitude and the frequency [GOTZ, 1987, Lehmann and Dickinson, 1998, Shih and Buchanan, 1971] as well as the speed and the timing of the wing rotation [Dickinson and Gotz, 1993].

Other insects such as dragonflies and damselflies accurately modulate their wing flapping amplitude, flapping frequency, inclination of the stroke plane with respect to the flight path, downstroke to upstroke ratio and phase difference between the forewings and the hindwings to control body motion through the air [Ruppell, 1989, Wakeling and Ellington, 1997]. The bio-inspired experimental studies, systematically, studied correlation between the wing kinematics and the flight force production [Sane and Dickinson, 2002]. It is equivalently important to investigate how insect can effectively alter the wing kinematics. The flight muscles in the insect thorax are responsible for these changes. 
The muscles are the motor of the locomotion. In the nature, flying insets employ two types of neuromuscular systems for flight. In most of them, such as diptera, the flight muscles are organized into two anatomically, physiologically, and functionally distinct groups: the large asynchronous indirect muscles that generate the power required for the flight, and the small synchronous direct muscles that are responsible for steering the maneuvers [Dickinson et al., 1998]. The bulk of the space inside the thorax of the insect is filled with the power muscles which are not directly connected to the base of the wing. They rather distort the thorax and an elaborate hinge transforms that to the wing kinetic energy. Power (indirect) muscle are arranged in two antagonist groups: large dorsal longitudinal fibers (six pairs in flies) and (seven pair in flies) dorsal ventral fibers. Each fiber is a single syncitial cell innervated by one motor axon [Dickinson et al., 1998]. Contraction in asynchronous muscles are initiated and terminated by the process of stretch activation and shortening deactivation [Huxley, 1974]. During flight, the contraction of each antagonist muscle group provides the required mechanical stretch to activate the other. Stretch activation property of flight muscle has been first discovered by Pringle in 1949 [Pringle, 1949]. For a muscle that is cyclically contracting, stretch activation and shortening deactivation cause stress to lag behind strain and ensures positive output work within each cycle. The firing rate of neuron motors innervating asynchronous muscles is an order of magnitude smaller than contraction frequency [Dickinson et al., 1998]. There are also several pairs of the direct muscles in the thorax, which through their action on the wing hinge, they affect how the mechanical power from the power muscles transfers to the wing kinematics [Dickinson et al., 1998]. In order to function these muscles don't have to generate high levels of mechanical power. At high frequencies the predominate effect of stimulation was a tonic increase in dynamic stiffness. The change in the wing kinematics then alters the flight forces and allows steering and maneuvering. Employing high speed videography techniques and electromyograms (EMGs), researchers were able to correlate the muscle activities to the wing kinematics changes by recording the muscle activity and the wing kinematics simultaneously. Today's technology allows conducting these measurements in free flight [Wang et al., 2008, Springthorpe et al., 2012] as well as tethered [Tu and Dickinson, 1996, Balint and Dickinson, 2001].

Flight muscles of Odonata (such as dragonflies and damselflies) [Pfau, 1986] and Blattaria (such as cockroaches) [Tiegs, 1955] are different. They use direct muscles to generate downstroke. In addition to their role in rotating the wing downward in downstroke, the direct flight muscles also 
affect the wing rotation about its longitudinal axis [Dudley, 2002]. Pronation refers to the rotation of the leading edge downward, whereas supination is the rotation in the opposite direction. For instance, in locust, the nearly simultaneous contraction of the basalar and subalar muscles generates the downward motion of the wing in downstroke, while the variation in the contraction of the basalar muscle causes the wing pronation [Wolf, 1990]. In beetle subalar muscles regulate wing's angle of attack as well as other more subtle changes in the wing kinematics [Kammer, 1971]. Odonata also posses limited dorsolongitudinal musculature that assist with wing depression, although they are generally considered to utilize direct flight muscle [Pfau, 1986]. Muscle function during both downstroke and upstroke are therefore widely variable among winged insects [Dudley, 2002].

Taken together, investigations show that the biomechanics of the wing hinge as well as the neuro-muscular control of the wing motion is quiet complex. For any given taxon, only a few flight muscles were studied thoroughly. There is especially a lack of investigation of the muscle action in free flight. It is not known to what extent insects are capable of independently controlling the distinct wing kinematics parameters. In addition, there is far less known about the correlation between the neuromuscular control (muscle activities) and the resultant aerodynamic force output. Only a few studies investigated this matters, for instance [Springthorpe et al., 2012].

Although insects are capable of actively controlling the wing kinematics, the mechanics of the wing and its hinge allows the wing to passively flip in response to the aerodynamic, inertial and the elastic torques. There is a growing body of evidence that supports passive maintenance of the wing angle of attack [Ishihara et al., 2009] as well as the passive wing rotation at stroke reversal [Bergou et al., 2007, Ennos, 1988] in hovering flight. In addition, it was suggested that fruit flies may combine active and passive wing kinematic changes in order to perform a turn maneuver. In this theory, the asymmetry in the bilateral wing pitch angle is indirectly induced by actively changing the properties of the elastic material in the wing hinge [Bergou et al., 2010]. Therefore, understanding the underlying physics of the wing kinematics control as well as the overall dynamics of aerial maneuvers is not possible without comprehending the interactions of the wing and its environment. 


\subsection{Development of flapping Micro Air Vehicles (MAVs)}

Flapping MAVs are potentially capable of achieving exceptional flight agility and maneuverability as that of flying insects. They are also low cost compared to the large air vehicles and small in size which makes them suitable for flying in inaccessible environments. The reciprocatory nature of the wing motion allows implementing simple and effective wing actuation techniques with no need for heavy engines or motors. Using a network of small, agile and potentially disposable MAV, rather than larger and more complex ones, allows better mission coverage and lowers the failure risk. During the past several decades there have been multiple research projects aiming to develop flying robots with flapping wings, such as butterfly-like robot designed and manufactured in University of Tokyo [Tanaka et al., 2005]. Mechanical Flying Insect (MFI) project is another project based at U.C. Berkeley. The wings of MFI are piezoelecric actuated and flappinmg frequency can be increased to $275 \mathrm{~Hz}$ for high lift generation. Single wing length is $8 \mathrm{~mm}$ and can generate up to $1400 \mu N$ of lift (Figure 2.3A). "RoboBee" is the smallest man-made flapping wing robot designed and manufactures at Harvard (Figure 2.3B). Its wingspan is $3 \mathrm{~cm}$ and it is capable of tethered flight. the wings are actuated by piezoelectric and the wing hinge is plastic to allow passive wing rotation at stroke reversal. Each wing can be controlled independently [Wood, 2007, Ma et al., 2013]. Delfly at University of Delf is another flapping wing robot inspired by dragonfly. It has a camera onboard and is remotely controlled. It uses DC electric motor for transmission (Figure 2.3C)[de Croon et al., 2012]. Nano Hummingbird at AeroVironment is another flapping wing robot inspired by hummingbird. It also uses DC motor for wing transmission (Figure 2.3D) [Keennon et al., 2012]. 




Figure 2.3: Flapping Micro Air Vehicles. State of the art flapping wing Micro Air Vehicles. (A) MFI, (B) RoboBee, (C) DelFly, (D) Nano Hummingbird.

The challenge of developing an insect size MAV spans across many disciplines. One of the major challenges is low cost and effective wing actuation. Recent developments have shown promising results in application of piezoelectric materials for generating reciprocatory motion of the wings. These actuators are commonly designed to operate at resonance, which in theory allows inertial and restoring elastic forces to balance. As the size shrinks, standard macro-scaled machining techniques can not be applied to the manufacturing. Furthermore, as the size decreases losses such as friction and electro-static become dominant and the traditional bearing joints can not be used anymore . Similar argument cam be applied to the transducers which their effectiveness decreases by decrease in size due to the friction and limits on the current density [Wood et al., 2012]. Another aspect of the design that is significantly affected by the size is the control. Larger vehicles can carry 
sensors, computers and complex actuators. Insect size MAV have a significantly reduced payload capacity compared to the larger air vehicles. Therefore, the control challenges are centered about the stabilizing and controlling the flight with minimum sensory input and computation cost. 


\section{Chapter 3}

\section{Dynamic coupling in dragonfly free flight}

\subsection{Introduction}

Aerial maneuvers of different insect species are studied widely in the last decades and many aspects of maneuverability in insect flight are explored. Although insects are capable of performing a variety of aerial maneuvers, technical challenges in acquiring accurate three dimensional data limited diversity and depth of our knowledge about flapping flight in nature. Recently high speed photogrammetry technology together with different auto-tracking and reconstruction techniques [Ristroph et al., 2009, Koehler et al., 2012, Wagner, 1986] inspired more comprehensive and accurate studies on the different aspects of the insect flight. Aerial maneuvers of different insects have been recorded and detailed body and wing kinematics were measured [Ribak and Swallow, 2007, Wang et al., 2003]. In free flight, predation and evasive flights have been studied which revealed significant information about population dynamics and individual fitness [Combes et al., 2012, McLachlan et al., 2003] But yet most of the studies on the dynamics of the insects' aerial maneuvers are either limited to tethered flight or narrowed down to the turns that are modeled mainly by conventional "banked turn" model. Similar to the fixed wing aircrafts, in banked turn, insect redirects flight force vector by banking to the direction of turn. Studies revealed that many insects and birds use this strategy for performing aerial turns such as fruit fly [Fry et al., 2003], locust [Berger and Kutsch, 2003], dragonfly [Alexander, 1986], fruit bats and cockatoos [Hedrick and Biewener, 2007, Iriarte-Diaz and Swartz, 2008]. Banked turn is a semi-2D model mapped to the three dimensional nature of these maneuvers, derived from decoupling governing equations of motion (EOM). However some studies recorded aerial maneu- 
vers of insects in which rotational velocities are simultaneously high [Schilstra and Hateren, 1999]. Dynamics of these maneuvers cannot be modeled without considering dynamic coupling effects. But then it's not clear what role these couplings play in the maneuver and why insects employ both kinds of strategies. Since flight is an expensive type of locomotion, insects (and birds) have learned to use their wings, body and legs together to increase efficiency of their flight and enhance stability and maneuverability [Dudley, 2002]. Although wings play the most important role in the flight, as the main source for aerodynamic forces and torque generation, insects know how to use their abdominal deflections to enhance flight stability [Dyhr et al., 2013]. Several studies have shown that leg extensions play an important role in stabilizing the flight as well [Gotz et al., 1979]. However, in our study of dragonfly's turn takeoff we have shown another mechanism that they use to enhance maneuverability, together with using their four wings and abdominal deflections (abdominal deflections in these maneuvers are investigated separately in another study). Since dragonfly is taking off and changing the flight direction at the same time, extra mechanisms to execute the maneuver successfully and efficiently are possibly required. In this chapter, we start with modifying governing equations of rigid body rotation consistent with wing-body configuration of dragonfly. Individual terms are then discussed and named. One of these terms, called DCA (Dynamic Coupling Acceleration) which represents strength of the coupling in dynamics. More discussion on the physical interpretation of this term in the case of dragonfly's maneuver is provided later in this work (in discussion section). Afterward, a simple conceptual model is represented and used to examine effectiveness of the DCA term in manipulating the orientation. Argument is followed by representing free maneuvering flight measurements and calculating contribution of DCA term. Correlation of pitch and yaw velocity with this acceleration term is then examined. We finally discussed that dragonfly's body reorientation maneuver is rather a three dimensional phenomena than planar and decoupling EOM may result in inaccurate models. Later on in the discussion, we compared two maneuvers of the same dragonfly and showed how difference in the acceleration throughout the course of maneuvers can be explained by including DCA term in the analysis. We concluded that in spite of the more complex appearance of trajectories of 3D maneuvers compared to planar, dragonflies are able to perform a variety of 3D maneuvers employing a simpler control strategy by controlling the DCA term. 


\begin{tabular}{ccccc}
\hline Dragonfly & Year & Gender & Weight $\mathbf{( m g )}$ & Flapping frequency $\mathbf{( H z )}$ \\
\hline B & 2010 & Male & 265 & 42 \\
G & 2011 & Female & 83 & 26 \\
R & 2011 & Female & 166 & 33 \\
Y & 2011 & Female & 119 & 26 \\
\hline \hline
\end{tabular}

Table 3.1: Morphological data base of dragonflies in experiment.

\subsection{Dragonflies}

Dragonflies were captured during the summer months of 2010 and 2011 in Dayton, OH, and were identified as Eastern Pondhawk, (Erythimus Simplicicollis) species. Before performing the experiment they were stored outdoor in specific containers which were open to sunlight and air. This ensured that they remained active in the relatively colder camera room. Table 1 includes morphological data for each of the case studies and initial and final orientation of the body. For the sake of convenience we named the dragonflies as blue (B), green (G), red (R) and yellow (Y).Dragonfly $\mathrm{B}$ is a male and all other three are females.

\subsection{Experimental setup}

The photogrammetry setup used for dragonfly image collection consists of three synchronized Photron FASTCAM SA3 60K high-speed cameras with 1024x 1024 pixel resolution. They were aligned orthogonal to each other on an optical table and operated at $1000 \mathrm{~Hz}$ with at least a 1/20000 sec. shutter speed to capture the dragonfly flight videos. The dragonflies were illuminated by 3 halogen photo optic lamps (OSRAM, 54428). The cameras were positioned 1.5 meters away from the insects, giving a depth of field of 3-4 body lengths in all directions depending on the size of the specimen [Koehler et al., 2012].

Dragonflies take off voluntarily from a rod or a paper stool in the middle of the shooting area. The 3D surface reconstruction technique is then [Koehler et al., 2012] applied to the raw output from the high speed cameras. We prepared a dragonfly-body template mesh which was animated by aligning the projection of its outer border to the silhouette of the body in the images. Body orientation and location in each video is measured by matching the wing roots' plane of the body mesh to the insect body. We filmed each dragonfly several times and among those we chose the videos in which at 
least two projections offered good quality images. Orientation of the wing roots plane in 3D space defines body orientation during the maneuver. Normal vector to this plane is used to define the roll angle. The internal consistency of the data further corroborates that the measurements were performed with adequate precision. Furthermore, our measured values were in good agreement with previously reported data on angular velocities of dragonflies in turn [Wang et al., 2003]. Detailed descriptions about the reconstruction method and accuracy can be found in [Koehler et al., 2012].

\subsection{D surface reconstruction}

The 3D surface reconstruction technique [Koehler et al., 2012] is applied to the raw output from the high speed cameras. We prepared a dragonfly-body template mesh which was animated by aligning the projection of its outer border to the silhouette of the body in the images. Body orientation and location in each video is measured by matching the wing roots' plane of the body mesh to the insect body. Sometimes, varying distances of the insect in free flight from each camera causes the images obtained to be out of scale or defocused. For the sake of accurate reconstruction we film each dragonfly several times. Among these we chose videos in which at least two projections offered good quality image. Orientation of the wing roots plane in 3D space defines body orientation during maneuver. Normal vector to this plane is used to define roll angle. The internal consistency of the data further corroborates that the measurements were performed with adequate precision. Furthermore, our measured values were in good agreement with previously reported data on angular velocities of dragonflies in turn [Wang et al., 2003]. Detailed descriptions about the reconstruction method, limitations via image quality and insect shape are discussed in [Koehler et al., 2012].

\subsection{Description of the turn takeoff maneuver}

This terminology is used to describe the maneuver in which dragonfly redirects the flight while it's taking off at the same time. In addition to turn a significant elevation in center of mass, 1-2 body lengths, were observed during performance of this maneuver. Fig. 1 demonstrates a typical turn takeoff. Three sample frames taken directly from the high speed camera output (Fig. 1A). Fig.s 1B-C show the reconstructed body at 5 snapshots of the same maneuver (as in figure 1A) in side and top views. This kind of flight typically starts with pitching up rotation, which lasts for a about 
a wingbeat long, and is followed by pitching down. Based on this observation, in our analysis, the maneuver is divided into two phases.

A

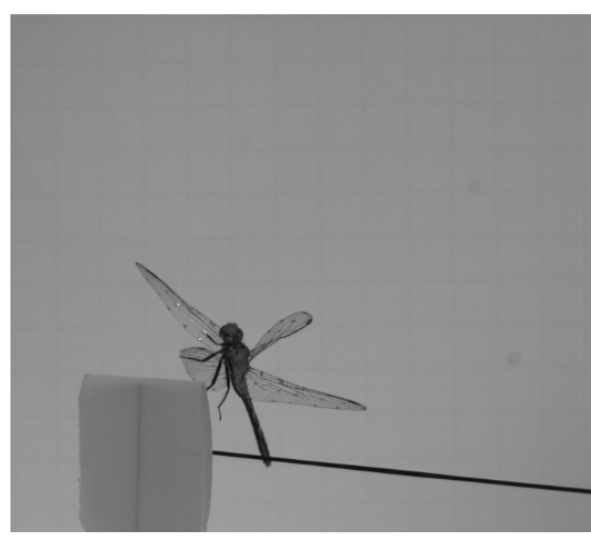

$\mathrm{t}=0 \mathrm{~ms}$

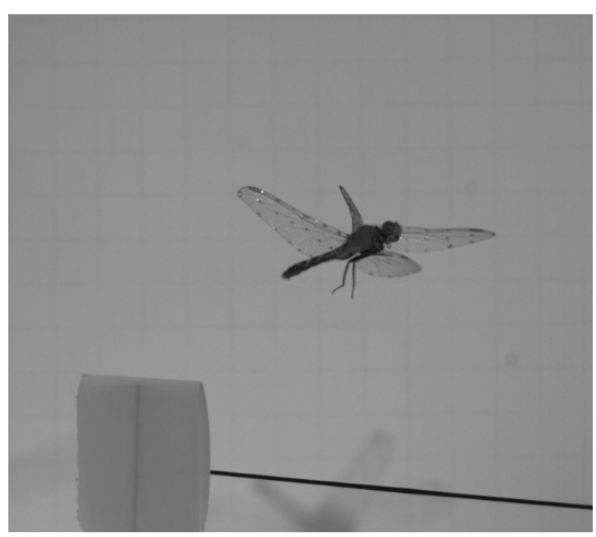

$\mathrm{t}=50 \mathrm{~ms}$



$\mathrm{t}=100 \mathrm{~ms}$

$\mathrm{B}$

$\mathrm{C}$


Figure 3.1: Dragonfly turning takeoff. (A) Sample of 3 snapshots of a typical turn takeoff maneuver. (B) and (C) show reconstructed body surface of the same dragonfly in Fig. 1A. In a typical turn takeoff maneuver center of mass elevates about 1 to 2 body lengths and turn angle is about 80120 degrees. Corresponding time is shown next to each snapshot.

\subsection{Calculation of body moment of inertia}

Moment of inertia (MOI) is a measure of the resistance of a body to rotation and because insect body morphologies are not comprised of regular geometric shapes, their body shapes are ap- 
proximated in order to calculate the MOI [Zeyghami and Dong, 2012, Zeyghami and Dong, 2015, Combes and Dudley, 2009, Hedrick and Daniel, 2006, Taylor and Thomas, 2003]. On the contrary, in this paper, we accurately calculate the moment of inertia tensor with the confidence of reliability in all degrees of freedom. The moment of inertia for the dragonfly was obtained from a 3D model constructed in MAYA (Autodesk, San Rafael, CA, USA) based on images of a common species; Erythimus simplicicollis, found in North America. Two images; a top and side view are necessary for accurately constructing the 3D model. The non-dimensional body morphological data obtained from the dragonfly model is included in Table 3.2. The MOI data is non-dimensionalized in Table 3.2 by $M / L^{2}$, where $\mathrm{M}$ is the body mass $(252.52 \mathrm{mg})$ and $\mathrm{L}$ is the body length $(43.95 \mathrm{~mm})$.

A
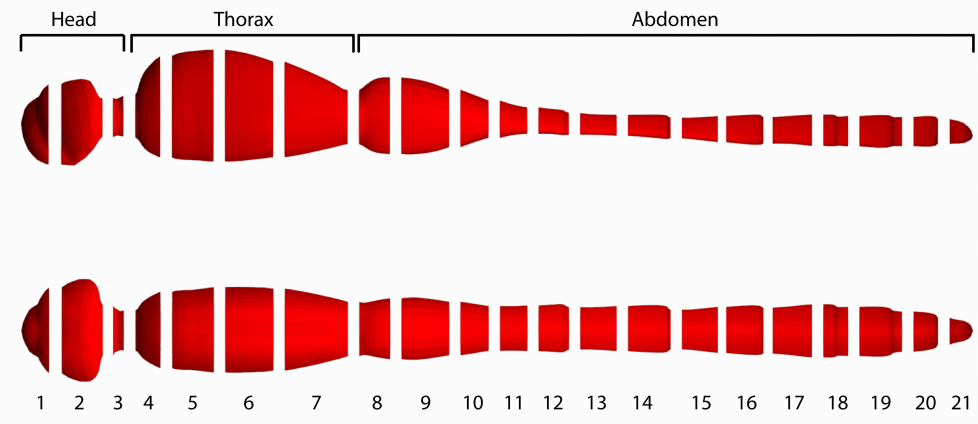

B
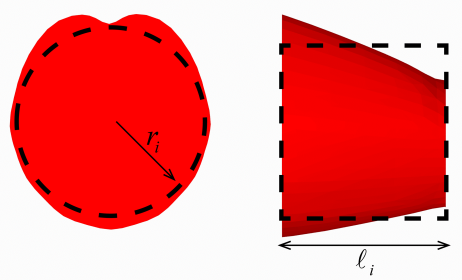

Figure 3.2: Dragonfly model for MOI calculations. (A) depicts a sliced dragonfly model. The model is sliced into 21 pieces. Slices 1-3, 4-7, 8-21 make up the head, thorax, and abdomen, respectively. (B) shows the approximation of an $i^{\text {th }}$ slice as a cylinder having a volume equivalent to the slice. $r_{i}$ shows the corresponding radius of the approximated cylinder and $l_{i}$ shows the length of the cylinder. The length of the cylinder is equal to the length of each slice.

For analysis, the 3D model was sectioned into 21 pieces as shown in Fig. 2. Slices 1-3, 4-7 
and 8-21 make up the head, thorax and tail regions respectively. We established that 21 slices provided enough accuracy for our study. However, since a computer algorithm is responsible for the calculation of MOI, the number of sections can be infinitely increased and the thickness of each section need not be uniform.

\begin{tabular}{|c|c|c|c|c|c|c|c|}
\hline & Section \# & $l_{i}$ & $V_{i}$ & $I_{x x}{ }^{*} \times 10^{4}$ & $I_{y y}{ }^{*} \times 10^{4}$ & $I_{z z}{ }^{*} \times 10^{4}$ & $I_{x z}{ }^{*} \times 10^{4}$ \\
\hline \multirow{3}{*}{ Head } & 1 & $3.93 \mathrm{E}-02$ & $2.00 \mathrm{E}-04$ & \multirow{7}{*}{15.00} & \multirow{7}{*}{97.00} & \multirow{7}{*}{96.00} & \multirow{7}{*}{-0.44} \\
\hline & 2 & $5.71 \mathrm{E}-02$ & $6.24 \mathrm{E}-04$ & & & & \\
\hline & 3 & $6.81 \mathrm{E}-03$ & $3.69 \mathrm{E}-05$ & & & & \\
\hline \multirow{4}{*}{ Thorax } & 4 & $3.52 \mathrm{E}-02$ & $2.76 \mathrm{E}-04$ & & & & \\
\hline & 5 & $5.76 \mathrm{E}-02$ & 7.79E-04 & & & & \\
\hline & 6 & $6.63 \mathrm{E}-02$ & $9.03 \mathrm{E}-04$ & & & & \\
\hline & 7 & 8.66E-02 & $6.85 \mathrm{E}-04$ & & & & \\
\hline \multirow{3}{*}{ Inner tail } & 8 & $4.27 \mathrm{E}-02$ & $2.46 \mathrm{E}-04$ & \multirow{14}{*}{1.50} & \multirow{14}{*}{230.80} & \multirow{14}{*}{230.50} & \multirow{14}{*}{-6.80} \\
\hline & 9 & $6.59 \mathrm{E}-02$ & $4.10 \mathrm{E}-04$ & & & & \\
\hline & 10 & $3.91 \mathrm{E}-02$ & $1.40 \mathrm{E}-04$ & & & & \\
\hline \multirow{11}{*}{ Outer tail } & 11 & $3.76 \mathrm{E}-02$ & $7.71 \mathrm{E}-05$ & & & & \\
\hline & 12 & 4.19E-02 & $6.70 \mathrm{E}-05$ & & & & \\
\hline & 13 & $5.02 \mathrm{E}-02$ & $6.00 \mathrm{E}-05$ & & & & \\
\hline & 14 & $5.86 \mathrm{E}-02$ & $8.12 \mathrm{E}-05$ & & & & \\
\hline & 15 & $4.93 \mathrm{E}-02$ & $6.46 \mathrm{E}-05$ & & & & \\
\hline & 16 & $5.11 \mathrm{E}-02$ & $9.45 \mathrm{E}-05$ & & & & \\
\hline & 17 & $5.40 \mathrm{E}-02$ & $1.01 \mathrm{E}-04$ & & & & \\
\hline & 18 & $3.43 \mathrm{E}-02$ & $7.16 \mathrm{E}-05$ & & & & \\
\hline & 19 & $5.89 \mathrm{E}-02$ & $1.16 \mathrm{E}-04$ & & & & \\
\hline & 20 & $3.42 \mathrm{E}-02$ & $5.05 \mathrm{E}-05$ & & & & \\
\hline & 21 & $3.32 \mathrm{E}-02$ & $1.91 \mathrm{E}-05$ & & & & \\
\hline
\end{tabular}

Table 3.2: Geometric Data of each slice of the dragonfly body. The non-dimensional length and volume of each slice of the dragonfly model are recorded. The non-dimensional MOI included in the table are non-dimensionalized by the body mass and length squared.

From the 3D model, we obtained the non-dimensional geometric center of mass as well as the volume of each slice. Afterward, each individual slice was approximated as a cylinder with a volume equivalent to the actual slice volume to quantify the contribution of the slices to the MOI. The equivalent cylinder has the same length as the slice and the circular cross-sectional area is oriented in the direction of the roll axis. The mass of each slice is computed by sectioning a real dragonfly into three pieces; head, thorax and abdomen. We assumed that density is constant across each section. The real insect body length is used to dimensionalize all the length quantities. From this dimensionalizaion, we obtain the dimensional volume of each slice and then multiply density by volume to obtain the mass of each slice. The tensor of MOI of the dragonfly was calculated by 
adding the MOI of each slice about the axes fixed at the center of mass of the body. $I_{\text {head }}^{\prime}, I_{\text {thorax }}^{\prime}$ , $I_{\text {tail }}^{\prime}$ are tensors of moment of inertia of the slices of the head, thorax, tail, respectively, about the axes originating from their own center of mass and fixed to them. $I_{\text {trans }_{i}}$ represents the tensor which contains the parallel axis theorem that enables us to calculate the effect of distance from the body center of mass to the center of mass of each individual slice.

$$
\begin{gathered}
I=\sum_{i \in n} I^{\prime}{ }_{i}+I_{\text {trans }_{i}} \quad n=\{\text { head,thorax, abdomen }\} \\
I_{i}{ }^{\prime}=\frac{m_{i}}{12}\left[\begin{array}{ccc}
6 r_{i}^{2} & 0 & 0 \\
0 & 3 r_{i}^{2}+l_{i}{ }^{2} & 0 \\
0 & 0 & 3 r_{i}^{2}+l_{i}{ }^{2}
\end{array}\right] \\
I_{\text {trans }_{i}}=m_{i}\left[\begin{array}{ccc}
y_{i}^{2}+z_{i}^{2} & x_{i} y_{i} & x_{i} z_{i} \\
y_{i} x_{i} & x_{i}^{2}+z_{i}^{2} & y_{i} z_{i} \\
z_{i} x_{i} & z_{i} y_{i} & x_{i}^{2}+y_{i}^{2}
\end{array}\right]
\end{gathered}
$$

\subsection{Frames of reference and coordinate systems}

Body orientation with respect to earth-fixed coordinate system is defined with three angles, bank $(\phi)$, elevation $(\theta)$ and heading angle $(\psi)$. Angular velocities, on the other hand, is usually studied about body axes and represented by roll velocity $(P)$, pitch velocity $(Q)$ and yaw velocity $(R)$. Rotational velocities in body and earth-fixed coordinate systems are related as follows:

$$
\begin{aligned}
& \dot{\varphi}=P+Q \sin (\varphi) \tan (\theta)+R \cos (\varphi) \tan (\theta) \\
& \dot{\theta}=Q \cos (\varphi)-R \sin (\varphi) \\
& \dot{\psi}=(Q \sin (\varphi)+R \cos (\varphi)) \sec (\theta)
\end{aligned}
$$

Body coordinate system is right handed and fixed at the body center of mass which is estimated to be in the middle of the four wing roots on the longitudinal body axis which points to the nose referred as $\mathrm{X}$-axis. The $\mathrm{Y}$-axis points toward the right wings and the $\mathrm{Z}$-axis points downward. 


\subsection{Equations of motion and effect of coupling term}

Rotational motion of any rigid flying body can be expressed as follows:

$$
\begin{aligned}
& \frac{d \vec{L}}{d t}+\vec{\omega} \times \vec{L}=\vec{M} \\
& \vec{L}=[I] \vec{\omega}
\end{aligned}
$$

$[I]$ is tensor of moment $\mathrm{f}$ inertia. On the right hand side of Equation 3.5 is vector of flight torque which in the case of insect's free flight is the same as aerodynamic torque. Inertial effects of the flapping wing also have minor contribution in creating torque on the body but because of very small wing to body mass ratio in dragonflies we ignored this term [Hedrick et al., 2009]. Inertial forces via fluid mass which is carried by wings are considered in aerodynamic torque term. The first term on the left hand side is rate of generation of angular momentum (by the means of external torque applied on the system). The second term on the left hand is the cross product of instantaneous rotational velocity vector and angular momentum. In two dimensions both these vectors are normal to the plane of motion and their cross product is zero. Therefore, when decoupling equation of motions in three spatial directions, this term is ignored. Although further discussion on the physical meaning and importance of this term is postponed to discussion section, we will briefly show how it will appear in definition of total acceleration. Governing equations of rigid body rotation, Euler equations, can be expanded as shown in Equation 3.6. On the left hand side of this equation there are configuration parameters such as mass and moment of inertia, along with inertial forces and torques.

$$
\begin{aligned}
& I_{x x} \dot{P}-I_{x z} \dot{R}-I_{x z} P Q+\left(I_{z z}-I_{y y}\right) R Q=L \\
& I_{y y} \dot{Q}+\left(I_{x x}-I_{z z}\right) P R+I_{x z}\left(P^{2}-R^{2}\right)=M \\
& I_{z z} \dot{R}-I_{x z} \dot{P}+\left(I_{y y}-I_{x x}\right) P Q+I_{x z} Q R=N
\end{aligned}
$$

$\mathrm{P}, \mathrm{Q}$ and $\mathrm{R}$ are rolling, pitching and yawing velocities and dot notation shows the first derivative with respect to time. These terms represent angular velocities about body axes. L, M and $\mathrm{N}$ are rolling, pitching and yawing torque and are referred to a "direct aerodynamic torque" in this paper. To get a clearer picture, we rearranged Equation 3.6 as below: 


$$
\begin{aligned}
& \dot{P}=\frac{L}{I_{x x}}+C_{P} R Q+D_{P}(P Q+\dot{R}) \\
& \dot{Q}=\frac{M}{I_{y y}}+C_{Q} P R+D_{Q}\left(R^{2}-P^{2}\right) \\
& \dot{R}=\frac{N}{I_{z z}}+C_{R} P Q+D_{R}(\dot{P}-Q R)
\end{aligned}
$$

For convenience we defined two matrices $\mathrm{C}$ and $\mathrm{D}$ which are defined based on the wing-body configuration as bellow;

$$
C=\left[\begin{array}{c}
C_{P} \\
C_{Q} \\
C_{R}
\end{array}\right]=\left[\begin{array}{c}
\frac{I_{y y}-I_{z z}}{I_{x x}} \\
\frac{I_{z z}-I_{x x}}{I_{y y}} \\
\frac{I_{x x}-I_{y y}}{I_{z z}}
\end{array}\right], \quad D=\left[\begin{array}{c}
D_{P} \\
D_{Q} \\
D_{R}
\end{array}\right]=\left[\begin{array}{c}
\frac{I_{x z}}{I_{x x}} \\
\frac{I_{x z}}{I_{y y}} \\
\frac{I_{x z}}{I_{z z}}
\end{array}\right]
$$

Since body-wing configuration is symmetric about the longitudinal axis then $\mathrm{Ixy}=\mathrm{Iyz}=0$. Note that if body-wing is symmetric about lateral plane (plane which divides body to upper and lower sections) $I_{x z}$ becomes zero similarly. Usually in insects wings are very light compare to body (in case of dragonflies less than $1 \%$ of body weight) and body shape is very close to cylindrical which results in smaller roll moment of inertia compared to moment of inertia about yaw or pitch axes. $I_{x z}$ and $I_{x x}$ terms are ignorable compared to $I_{y y}$ and $I_{z z}$. We accurately estimated body moment of inertia for dragonfly B (table 1A). To obtain moment of inertia of other individuals based on values obtained for dragonfly $\mathrm{B}$, we scaled these values by the ratio of product of mass and body length squared, assuming that body shape is pretty similar for all dragonflies of same species. Note that in calculating moment of inertia we assumed that wings are widely spread on the sides of the body in a plane that passes center of mass of the system. Our calculations, shows that governing dynamics of rotational motion of a dragonfly in turn maneuver reduces to,

$$
\begin{aligned}
\dot{P} & =\frac{L}{I_{x x}}+\frac{(\dot{R}+P Q)}{2} \\
\dot{Q} & =\frac{M}{I_{y y}}+P R \\
\dot{R} & =\frac{N}{I_{z z}}-P Q
\end{aligned}
$$

Second term on right hand side of Equation 3.9 is named "Dynamic coupling terms". Effect of the aerodynamic and dynamic coupling acceleration (DCA) terms in reorienting the body is separable if we measure rotational accelerations and velocities. DCA term at each time moment is just a function of rotational velocities and accelerations (Equation 3.9). Aerodynamic acceleration 
can be calculated by subtracting instantaneous coupling acceleration vector from the total rotational acceleration.

\subsection{Conceptual model}

Unlike two dimensional space, in three dimensions a maneuver can be performed in such way that the cross product of angular momentum and angular velocity is not zero. This implies that the angular momentum can be both generated and redistributed in the space. The aerodynamic torque produced by the insect wings generates angular momentum. In addition, the angular momentum vector can be tilted in the space due to existing angular momentum in the system. To investigate importance of the latter mechanism in dragonfly maneuver, we built a conceptual dynamic model based on the Equation 3.9. Magnitudes of configuration constants are calculated using the aforementioned method for the body moment of inertia. The wings are modeled as two point masses located $40 \%$ of the wing length apart from the wing's root. We then solved for pitch and yaw accelerations and consequently velocities as a function of pitch and yaw torques (inserted on the insect by fluid reaction to the wing's motion) and roll velocity. To investigate importance of the DCA term we assume that pitch and yaw torques are predefined and only DCA term is available for modification to change the course of the maneuver. Although within wingbeat oscillations in flight torque may affect the time course of the maneuver, for simplicity and without losing the generality of the model or conclusions, we assumed a constant pitch and yaw torque during the maneuver $\left(\tau_{\text {Pitch }}, \tau_{\text {yaw }}\right)$. On the other hand because DCA terms are direct functions of roll velocity, a fairly more complicated model has been used for roll velocity, represented as summation of "m" harmonic functions.

$$
P(t)=P_{o}+\sum_{i=1}^{m} P_{i} \sin \left(i \Omega t+\alpha_{P_{i}}\right)
$$

Sis the flapping frequency in Equation 3.10. There are a total number of " $3+2 \mathrm{~m}$ " parameters which should be fixe to describe yaw and pitch torques and roll velocity during the maneuver. Magnitude of aerodynamic torques is chosen consistently with the measured values from insects' flight. Keeping yaw and pitch torques unchanged we calculated body orientation in several simulated maneuvers changing specification parameters of roll velocity (Figure 3.3). Modulation in roll velocity modifies maneuver via modifying the DCA term. We simulated four cases by changing roll velocity 
amplitude. Reference maneuver is simulated without any roll velocity. In this case, aerodynamic torque results in more than 100o change in heading angle during a left turn (solid lines in Figure 3.3). In the next two maneuvers we increased roll amplitude to 2000 and then 3000 (o/s) compatible with measured roll velocity in insect's maneuvers. Last maneuver includes a roll to the left and then back to the right. For instance in the third simulation, dragonfly model ended up in a right turn although yaw torque was generated to turn body to the left. All four cases result in totally different final orientations although yaw and pitch torques are identical for all of them. Note that pitch angle is being affected as much as yaw. In the following sections we'll discuss how DCA term modified motion in real dragonflies' maneuvers.

A



B



C

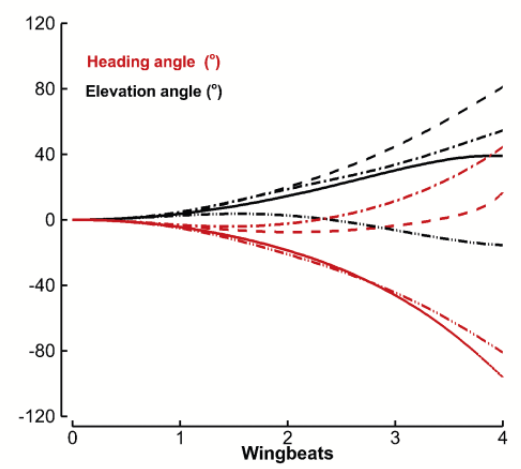

Figure 3.3: DCA conceptual model. We modeled a dragonfly body using moment of inertia inspired by real insect's measurements. Time history of yaw and pitch torque (A) are modeled as simple straight lines and they kept unchanged for all simulations. Roll velocity (B) is altered for each simulation by varying different specification parameters in Eqn 4. It turned out that higher frequency terms in roll velocity doesn't affect the course of maneuver significantly. Therefore we set $\pi \mathrm{i}=0,1,2,3$ to zero. $P_{4}$ is respectively $0,2000,-2000$ and $3000^{\circ} / \mathrm{s}$ for solid, dashed, dashed double dot and dashed dot lines in. Although aerodynamic torque was the same for all four cases, heading and elevation angles showed totally different patterns of change during the maneuver. Note that cycle frequency of roll velocity is written as a multiple of flapping cycle.

\subsection{Turning takeoff maneuver and patterns of change in body ori- entation}

Typically a turn takeoff maneuvers starts with a pitch up which lasts for about a wingbeat. It is then followed by a pitch down starting at the second wingbeat. Change in yaw angle varies from 
almost zero to more than $120^{\circ}$. Roll on the other hand, has a more complicated behavior. In some flights we observed a fast roll to the opposite direction of the turn at the beginning of the flight which then was followed by rolling back to the turn direction on the second wingbeat. Figure 3.4 shows the pitch, yaw and roll angles of the nine flights recorded in this study. Note that these angles are calculated by integrating pitch, roll and yaw velocities which are measured about body fixed axes. Therefore, initially all these angles will be zero. For consistency angles are plotted versus non-dimensionalized time which is defined as actual time over the length of the maneuver and varies between 0 and 1 . Average rotational velocities (of the 9 flights) are shown in the same figure as well. Rotational velocities of each maneuver were interpolated in 100 points along the nondimesionalized time course of the flight, and then averaged over the 9 maneuvers. Interestingly, yaw velocity remains almost constant during the flight and has smallest relative magnitude compared to the other two rotations. On the other hand, pitch velocity has a different behavior. For a small portion of the flight (first quarter of the flight) there is a significant pitching up velocity. At about the same time that insect starts to roll away from the turn direction, pitching velocity decreases and becomes negative. There is a noticeable concurrence between the location of the tops and deeps of the roll and pitch velocities. Roll velocity has a more complicated behavior, but it's evident that insect spends most of the flight time rolling away from the direction of the turn (roll velocity is mostly positive during the maneuver).

\subsection{Effect on angular velocities during the course of maneuver}

Trajectory of these flights were three dimensional rather than planar and all three rotational velocities were high in magnitude, unlike to the conventional yaw turns [Hedrick et al., 2009]. Therefore, it needed to be investigated whether dynamic coupling effects are negligible in governing dynamics of pitch and yaw rotations in these flights or not. Correlation of the yaw and pitch velocity at the end of each wingbeat with the time integral of the measured DCA term (coupling velocity), taken from the beginning of the maneuver to the end of the respective wingbeat, was examined (Figure 3.5). The correlation between pitch velocity and coupling velocity was found to be significant, $r^{2}=0.295$ and ANOVA $p<0.0002$. Yaw velocity on the other hand has shown a rather random correlation with coupling velocity. Dividing the data set into two sets of left and right turns, didn't help 
A
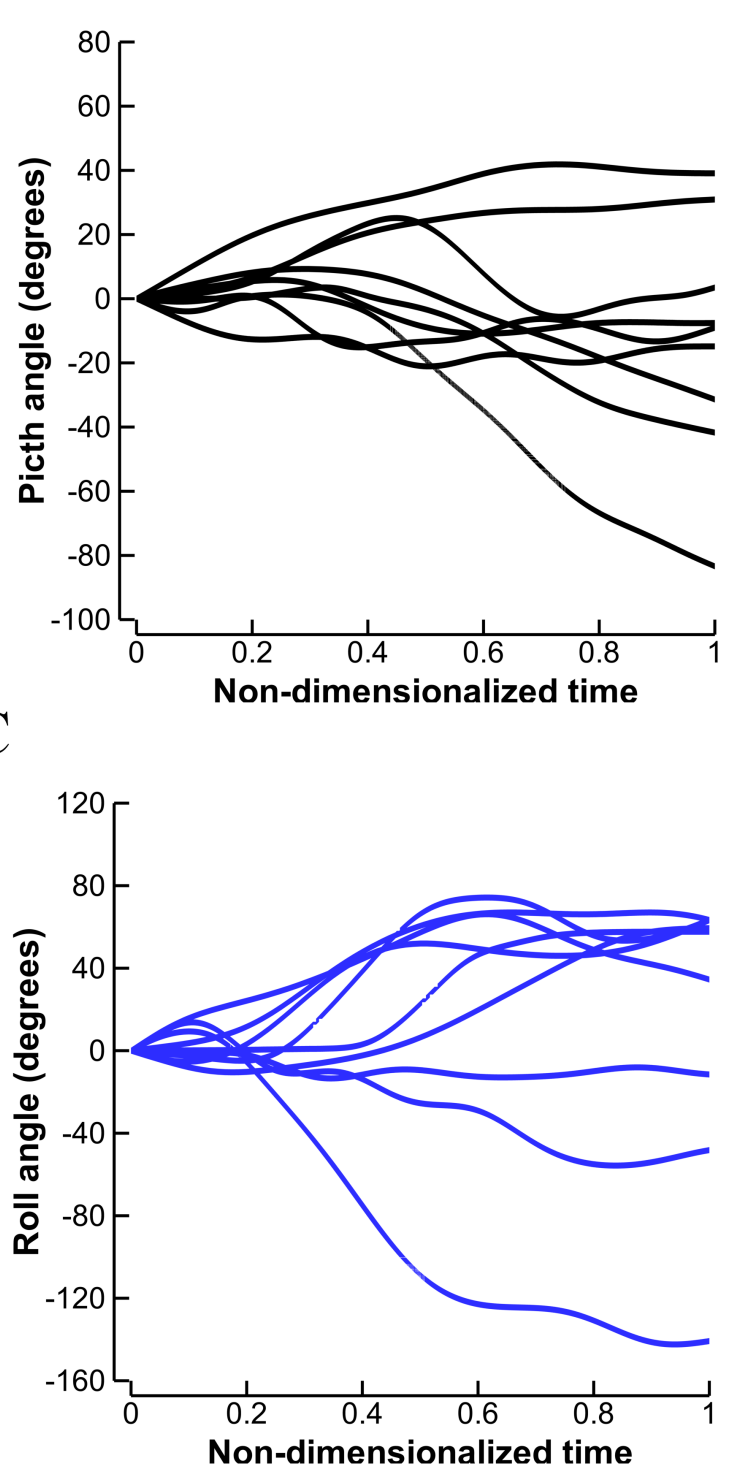

B
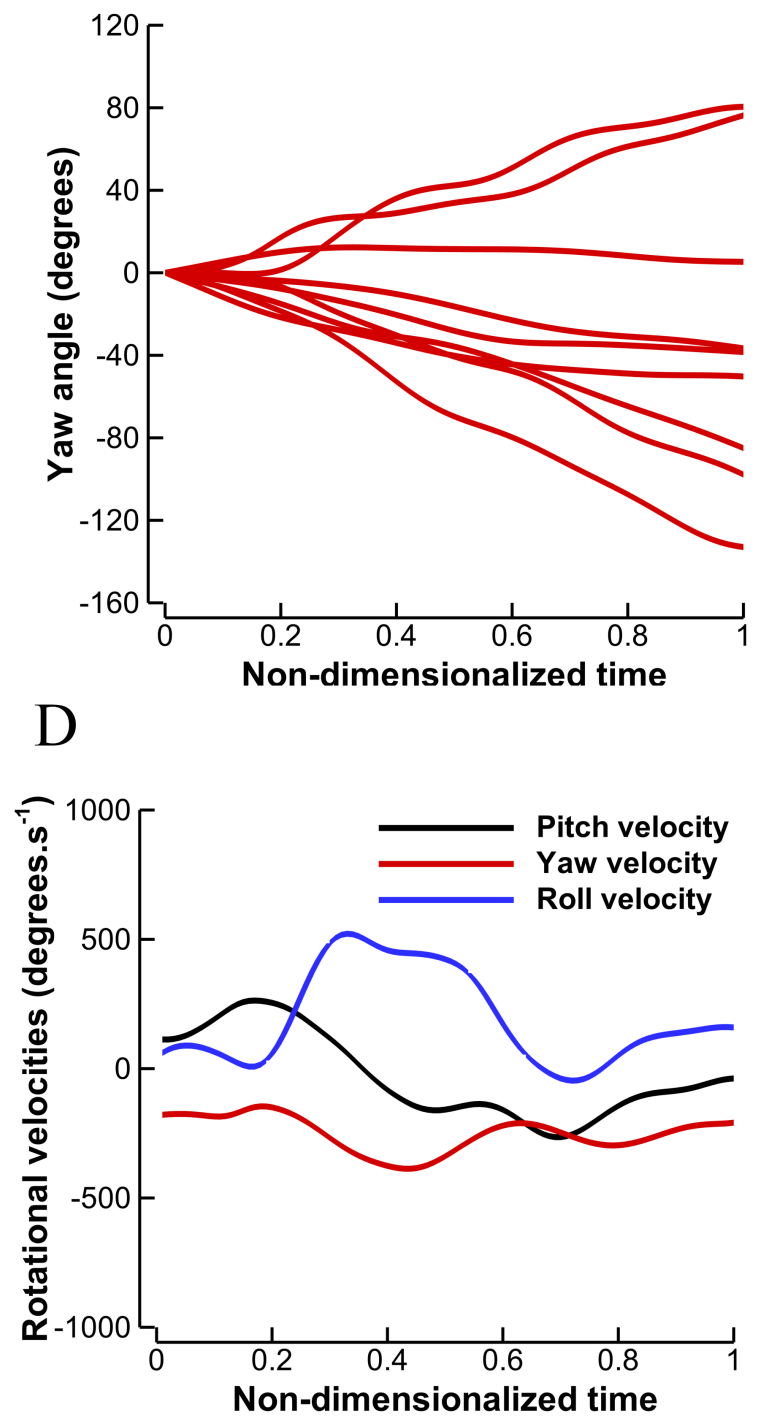

Figure 3.4: Body kinematics of dragonfly turning takeoff maneuver. Time history of pitch (A), yaw (B) and roll (C) angles in the 9 maneuvers. Average rotational velocities of the 9 maneuvers are shown in D. All rotational velocities are in the same order of magnitude. Yaw velocity remains almost constant during the flight. Pitch velocity, on the contrary, is positive for a portion of the flight and then starts to decrease and finally approaches zero. Roll velocity has a more complex behavior. Insect rolls away from the turn direction, during most of the time course of the maneuver. 
improving the trend and correlation was still seemed to be random for yaw velocity. This result however doesn't imply that the effect of the DCA can be neglected on the dynamics of yaw, but suggests that the insect is capable of performing the desired yaw rotation in spite of the effect of the dynamic coupling. We will further show that dynamic coupling can both enhance and reduce the yaw angle significantly. In contrast, conclusion is different for pitch. Strong correlation between pitch velocity and coupling velocity implies that dynamics of pitch in this flight is partially governed by the dynamic coupling effect. Our measurements indicate that about $30 \%$ of the pitch velocity can be provided by the DCA acceleration term. This infers that, compared to the case that all the pitching rotation is caused directly by the generation of pitch aerodynamic torque, performance of this flight requires less pitch torque. The strong correlation of pitch velocity with the coupling velocity may suggest a mechanism for controlling pitch velocity. Turn takeoff is a complicated maneuver in which the insect starts the flight by combining elevation of the center of mass together with changing the flight heading. Soon after the flight heading reaches the desired value (in 2-6 wingbeats), dragonfly continues with a climbing or a forward flight. Therefore control of this flight is expected to be more challenging and the insect may require extra mechanisms for maneuverability enhancement. Our results however suggest that insect would not need to recover the pitch angle completely by generating aerodynamic pitch (down) torque. Interactions between rotations in this flight, via dynamic coupling, can help to stabilize pitch rotations.

A

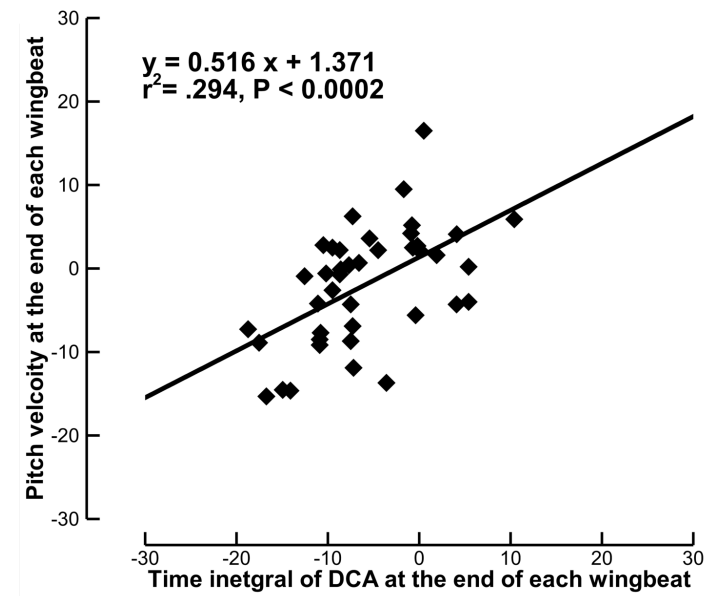

B

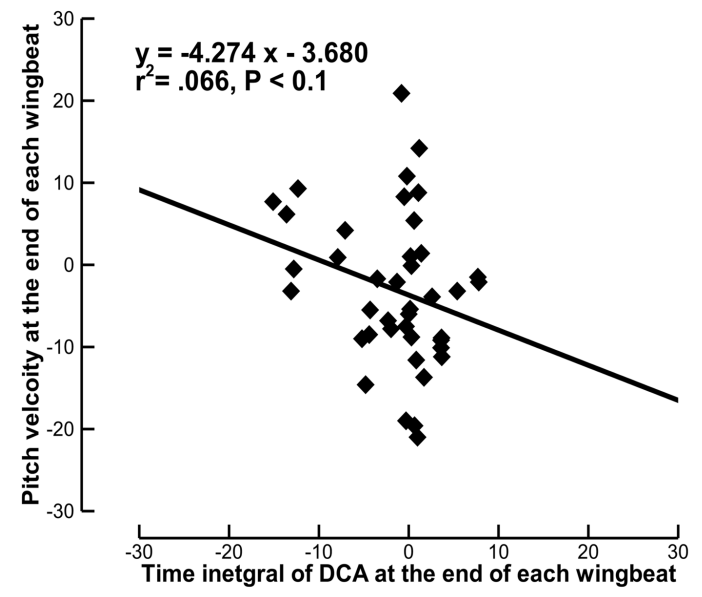

Figure 3.5: DCA in dragonfly free flight. Correlation of pitch (A) and yaw (B) velocities at the end of each wing stroke with time integral of DCA term. 
Although correlation of DCA with angular velocities is examined, it's still unclear how dynamic coupling can affect the course of the maneuver in the sense of orientation of the insect. In addition, we wanted to know why insect sometimes rolls to the opposite direction of the turn, as mentioned earlier, which is not compatible with the observations of the banked turn. Therefore orientation of the insect without the dynamic coupling effect, by integrating the aerodynamic acceleration (Eq.(2)) twice in time, was calculated. Fig. 5 shows the calculated orientation (dashed lines) together with the actual experimental measurements (solid lines) for two of the recorded flights. In most of the flights, DCA term was more important in manipulating pitch angle, in the first flight shown in Fig. 5, yaw angle was actually more affected by DCA. It's evident that although there was no correlation between the yaw velocity and its corresponding coupling velocity term, effect of the dynamic coupling effect on the dynamics of yaw cannot be safely neglected in all the cases. Our analysis on both pitch velocity and orientation supports this hypothesis. Based on whether the flight was a left or right turn, yaw angle was enhanced or reduced in these flights. On the contrary, DCA employed as a breaking mechanism for pitching, in all the recorded flights.

\subsection{Extra mechanism to enhance maneuverability}

In oppose to planar (2D) motion, in three dimensional space, angular momentum vector is not aligned with angular velocity except in very rare geometries or occasions. Therefore, system can change orientation by tilting angular velocity vector (moment free rotation). But more importantly, via modulating DCA, magnitude of this misalignment can be controlled and help modifying motion during the maneuver (Equation 3.11).

$$
\frac{d \vec{L}}{d t}=\vec{M}+\vec{L} \times \vec{\omega} \vec{L}=\left[\begin{array}{c}
I_{x x} P-I_{x y} Q-I_{x z} R \\
I_{y y} Q-I_{x y} P-I_{y z} R \\
I_{z z} R-I_{x z} P-I_{y z} Q
\end{array}\right]
$$

Angular momentum vector is represented in its general form in Equation 3.11, instead of the simplified form for dragonfly's rigid body model. That's to emphasize that body bending may also contribute similarly to the maneuver by modulating angular momentum vector (via changing moment of inertia) and consequently the DCA term and angular acceleration. Therefore in addition 


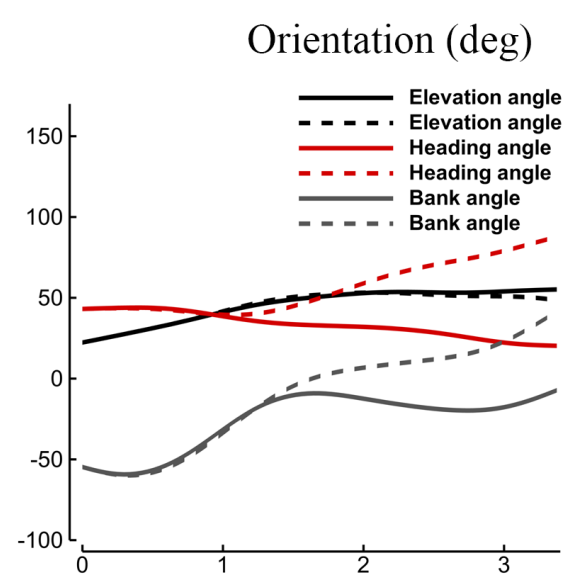

Cumulative orientation (deg)


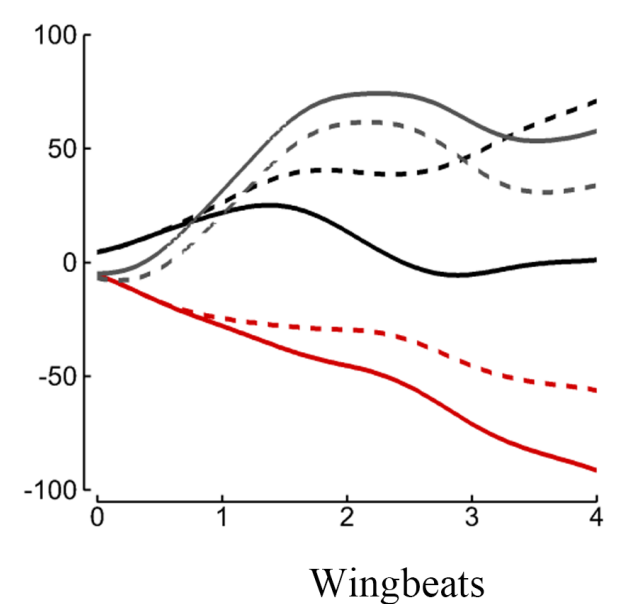

Angular velocity (deg/s)
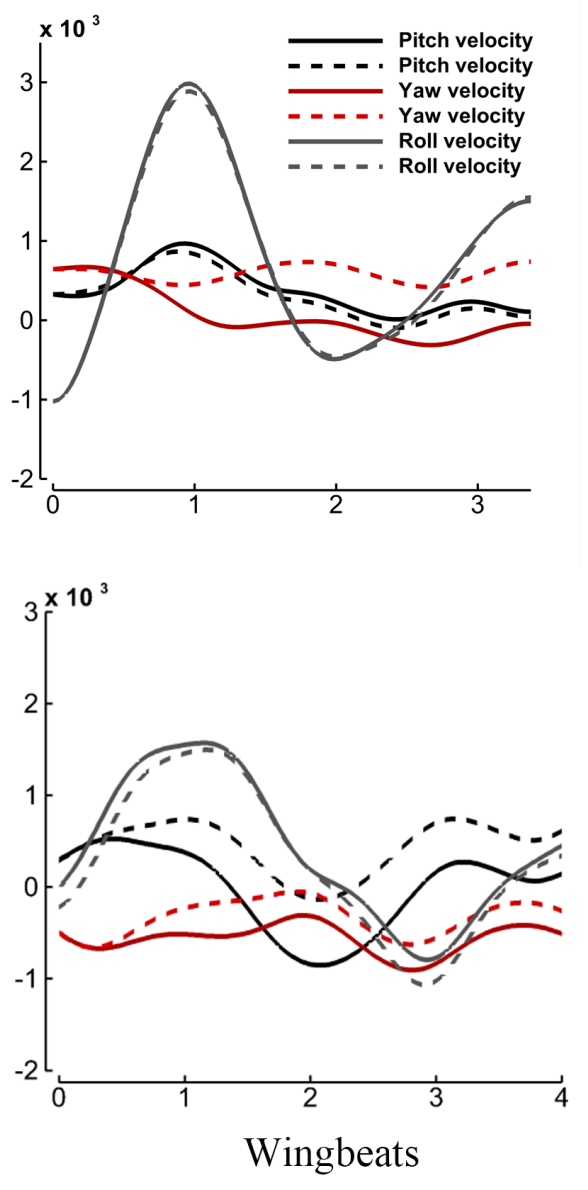

Figure 3.6: Effect of DCA on dragonfly body reorientation. Body orientation, cumulative body angular orientation and rotational velocities about body axes, corresponding to two samples of the recorded flights in this experiment, are shown in first, second and third columns, respectively. All graphs are plotted versus wingbeat number and columns share the same legend. Solid lines show real insect maneuver measurements and dashed lines represent how rotation will be if the coupling effect was neglected. 
to the aerodynamic torque, cross product of the instantaneous angular momentum and velocity can enhance the pitch or yaw, especially in the case of slender geometries like dragonfly's. Most importantly, strong correlation of the pitching velocity with the coupling velocity suggests existence of a non-direct stabilizing mechanism. Since DCA in pitch is product of the roll and yaw velocity, in both left and right yaw turns, dragonfly will be able to benefit from the dynamic coupling to decelerate pitching rotation. That's because in the second phase of the flight, when insect is rolling away from the direction of the turn, always this product will be negative and causes the body to pitch down. Subjectively, we interpreted this phenomenon as a sort of kinetic energy exchange between the rotations in which extra kinetic energy in pitch is rejected to the yaw which alternatively

enhances (or dampens) yaw. Note that not only insects use different mechanisms to rotate body along pitch and yaw axes but also their damping properties are different for each of these rotations [Cheng et al., 2011]. Therefore rejecting the extra energy from pitch to the yaw, which has higher damping, may act like a stabilizing mechanism.

\subsection{Comparison between two maneuvers of one dragonfly}

In two successive turn takeoff maneuvers, one of the dragonflies changed its heading about 90 and 120 degrees respectively (maneuver-I and II). If the effect of the coupling wasn't significant in these maneuvers, dynamics of the turn was expressible as bellow;

$$
\begin{aligned}
& I_{x x} \dot{P}=L \\
& I_{y y} \dot{Q}=M \\
& I_{z z} \dot{R}=N
\end{aligned}
$$

In this case, rotational acceleration is directly derived from the aerodynamics torque. In maneuver-II the dragonfly yawed less than $40^{\circ}$ while in the first one yaw exceeded $80^{\circ}$. The above model predicts that mean yaw torque in the first maneuver is more than 4 times bigger than the second one (based on mean angular accelerations and Equation 3.12). Similarly, mean pitch torque in first maneuver is predicted to be more than 16 times bigger than the second one. This means that for performing maneuver-I insect has to employ significantly higher levels of flight torques compared to the maneuver-II. On the contrary, our study suggests that main difference between these two 
maneuvers is generated via difference in the DCA term. We measured the mean DCA magnitude for each of the maneuvers and calculated mean aerodynamic acceleration based on Equation 3.5. Results are summarized in table 2. Our model suggests significantly lower ratios of aerodynamic torque between the two maneuvers (3.5 in pitch and 2.6 in yaw). Noting that duration of the maneuver-I is about half of the maneuver-II, these ratios are reasonable. Consistent with these results, our analysis also shows that body was forced by flight torque to pitch up about $60^{\circ}$ and yaw about $50^{\circ}$ in both maneuvers. Thus, although two maneuvers are very different in the sense of the trajectory and the time course of the body orientation, aerodynamic torques in yaw and pitch are very similar in both. Further modulation in motion is made possible via modulation in the DCA term.

\begin{tabular}{|c|c|c|c|c|c|c|c|}
\hline & & $\overline{\dot{Q}}\left(\mathrm{rad} / \mathrm{s}^{2}\right)$ & $\overline{\dot{R}}\left(\mathrm{rad} / \mathrm{s}^{2}\right)$ & $\overline{\dot{Q}}_{\text {aero }}\left(\mathrm{rad} / \mathrm{s}^{2}\right)$ & $\overline{\dot{R}}_{\text {aero }}\left(\mathrm{rad} / \mathrm{s}^{2}\right)$ & $\frac{\bar{M}_{I}}{\bar{M}_{I I}}$ & $\frac{\bar{N}_{I}}{\bar{N}_{I I}}$ \\
\hline \multirow{3}{*}{$\begin{array}{c}\text { Without } \\
\text { DCA }\end{array}$} & Maneuver-I & -273 & -14 & - & - & \multirow{3}{*}{17.0} & \multirow{3}{*}{3.5} \\
\hline & & & & & & & \\
\hline & Maneuver-II & -16 & -4 & - & - & & \\
\hline \multirow{3}{*}{$\begin{array}{l}\text { With } \\
\text { DCA }\end{array}$} & Maneuver-I & -131 & -55 & -141 & -70 & \multirow{3}{*}{3.5} & \multirow{3}{*}{2.6} \\
\hline & & & & & & & \\
\hline & Maneuver-II & 23 & 30 & -40 & 26 & & \\
\hline
\end{tabular}

Table 3.3: Estimation of the mean pitch and yaw aerodynamic torques with and without DCA term for two maneuvers of one dragonfly, referred to as Maneuver-I and II. Last two columns show the ratio of pitch and yaw torques of maneuver-I and II.

\subsection{Flight direction change with minimum effort}

Our investigation on the dragonflies' turning takeoff maneuvers suggested that these insects are able to minimize control effort and increase agility by adjusting rolling motion. We found out that roll motion is modified during the maneuver more often than the other two rotations. It was previously suggested that the rolling turn is done to rotate the aerodynamic forces vector consistent withe the banked turn model. In addition to that, our analysis shows that there exists a significant energy 
saving benefit in manipulating roll to transfer kinetic energy between the other two components of rotational motion.



Figure 3.7: B-spline. A third order B-Spline curve with four control points. The optimization problem moves the two red control points to manipulate the shape of the curve. The resulting curve can be used to define the time history of any dynamic variable.

To tease out the idea and to investigate whether dragonflies truly use this mechanism to enhance maneuverability and to save in controlling effort, we formulated an optimization problem which minimizes control effort during a desired maneuver. The optimization problem is solved in MATLAB. The initial and final orientation of the body are inputs to the problem. The optimization algorithm then finds the time history of changes in the body Euler angles which will ensure achieving the desired final orientation within a limited number of wingbeats (3 wingbeats). It also minimizes the aerodynamic pitch and yaw torque that are necessary to execute the maneuver. The cost function is defined as: 


$$
\begin{gathered}
\operatorname{cost}=C_{a}\left\{a b s\left(\psi-\psi_{f}\right)+a b s\left(\theta-\theta_{f}\right)+a b s\left(\phi-\phi_{f}\right)\right\} \\
+\left\{a b s\left(C_{\text {pitch }} \max \left(\bar{\tau}_{\text {pitch }}\right)+C_{\text {yaw }} \max \left(\bar{\tau}_{\text {yaw }}\right)+C_{\text {roll }} \max (\bar{P})\right)\right\}
\end{gathered}
$$


Figure 3.8: Optimized flight reorientation. (A) Time history of the measured body Euler angles for a dragonfly in a free flight turning maneuver. (B) The optimized time history of the body Euler angles for the same initial and final orientations as that measured in free flight.

where $C_{i}, i=a$, yaw, pitch, roll is the cost coefficient associated with accuracy of obtaining the final orientation, manipulations in the yaw torque, pitch torque and roll velocity, respectively. The heavy penalty on deviations of the obtained final orientation from the desired value (usually determined based on the measured orientation in free flight) ensures that the desired final orientation is achieved at the final solution. The defined problem treats roll velocity as the main control component and manipulates pitch and yaw moments for further control over the body orientation. This has been implemented by assigning a larger cost to the manipulations in the pitch and yaw torque when compared to the roll. The time history of roll velocity and the pitch and yaw moments within one wingbeat is modeled by three separate B-spline curves, each one with 4 control points, two of them were fixed (at two ends) and two are free to move (Figure 3.7). While there are other methods that one can generate an arbitrary continuous curve, such as a Fourier expression, a B-spline curve provide similar degree of freedom on the shpae of the curve with fewer variables (position of the control points). The optimization algorithm moves the control points in the 2D 
space, varying the time history of the associated term. At each iteration, the simplified EOM for a slender body (Equation 3.9) are solved to calculate rotational accelerations. The rotational velocities in the body coordinate system were then obtained by integrating the accelerations. Equation 3.4 is applied to calculate the Euler velocities and consequently the Euler angles. The output is the time history of the body Euler angles that allows reorientation of the insect from its initial to the final orientation. The optimized results ensure that the maneuver occurs within a limited number of wingbeats requiring minimum magnitude of pitch and yaw torque. The results are shown in Figure 3.8 and are compared to the measured Euler angles in free flight. Note that there are at least as many solution to this problem as there are ways to connect two points in space. The patterns of the change in the body Euler angles that are obtained from optimization resemble those measured in free flight (Figure 3.8), implying that the path insect takes to execute the maneuver allows it to use minimum aerodynamic torque.

\subsection{Acknowledgement}

The 3D surface reconstruction of the body is performed by Kuo Gai. 


\section{Chapter 4}

\section{Cicada flight mechanics}

\subsection{Introduction}

Cicadas are unique flying insects known for their long development period and large body size [Fry et al., 2005]. While a number of studies on the lifestyle and ecology of cicadas have surfaced in the scientific community, there is a lack of data on the flight characteristics of these insects [OBERDORSTER and Grant, 2007]. Periodic cicadas which emerge every 13 or 17 years are clumsy fliers which fly slowly and over short distances [Karban, 1981]. Conversely, annual cicadas are better fliers and show relatively higher flight speeds and predator-evasive behavior [OBERDORSTER and Grant, 2007]. Compared to insects with superior maneuverability such as fruit flies and dragonflies, cicadas have a large wing loading; meaning that for a unit wing area, a larger body mass needs to be carried (Table 4.1). While wing loading is known to inversely affect the flight performance [Ellington, 1991], our observations show that cicadas are capable of executing various aerial maneuvers. In voluntary turns recorded in this experiment, the average speed of the heading change was about $1000-1500 \mathrm{deg} / \mathrm{s}$ which is at about the same range as that of other insects [Hedrick et al., 2009].

The capacity to change the flight trajectory is essential for aerial locomotion and survival of flying animals. To adjust the flight course, flying animals modulate the magnitude and orientation of aerodynamic force by altering their wing kinematics. Highly maneuverable insects such as dragonflies [Alexander, 1986] and fruit flies [Fry et al., 2003] are capable of adjusting the wing stroke plane angle as well as the orientation of the wing in this plane to achieve exceptional control 


\begin{tabular}{clllllll}
\hline Insect & Fruit fly & Dragonfly & Damselfly & Hawk moth & Cicada & Bumble bee & Hummingbird \\
\hline Wing loading $\left(\mathrm{kg} / \mathrm{m}^{2}\right)$ & 0.25 & 0.1 & 0.14 & 0.89 & 1.5 & 1.65 & 2.9 \\
\hline
\end{tabular}

Table 4.1: Wing loading of several species of flying animals.

over the aerodynamic force. This is mostly achieved by manipulating the wing angle of attack [Bergou et al., 2010]or velocity [Fry et al., 2005] which leads to changing the magnitude and direction of the aerodynamic force. The ability to change the force direction relative to the body allows these insects to generate aerodynamic torque about the center of mass for body reorientation. While this enhances the maneuverability of a flying animal, it imposes complexity to the wing biomechanics as well as the control system of the flight [Ros et al., 2015]. Alternatively, flying insects and birds change the flight trajectory while maintaining the direction of aerodynamic force relative to their bodies. In these maneuvers, animals rely on whole-body rotations to redirect the force in the global frame. This strategy is referred to as force vectoring [Ros et al., 2015] and is widely observed in banked turns of insects [Muijres et al., 2014], bats [Iriarte-Diaz and Swartz, 2008] and birds [Hedrick and Biewener, 2007, Ros et al., 2015]. It was argued that force vectoring allows minimal modulations of the wing motion relative to the body [Ros et al., 2015]. While this is beneficial for simplifying the wing biomechanics, some degree of control over the aerodynamic force direction relative to the body is essential for stability and maneuverability [Ros et al., 2015].

Here, we studied a variety of flights of cicada (Tibicen linnei) to examine the extent to which the aerodynamic force is modulated for achieving this range of flights. Using high speed videography, different flight modes of cicada including forward flight, pitching up and down, banked turn, Immelmann turn, vertical takeoff and spin were recorded. Applying a 3D surface reconstruction technique, the motion of wings and body were tracked. In the voluntary turns recorded in this experiment, the average speed of the heading change was about $1000-1500 \mathrm{deg} / \mathrm{s}$ which is at about the same range as that of other insects [Hedrick et al., 2009]. The orientation of the aerodynamic force relative to the body was calculated using a fully-validated quasi-steady model. Our results indicate that the orientation of the half-stroke-averaged aerodynamic force remains invariant among all flights. Cicadas achieved a wide variety of flights by modulating the force relative to the body in a narrow range.

While a number of studies on the lifestyle and ecology of cicadas, especially periodic cicada, have 
recently surfaced in the scientific community [Williams and Simon, 1995], there is a lack of data on the flight characteristics of these insects [OBERDORSTER and Grant, 2007]. This work presents a comprehensive and accurate measurement on cicada free flight. These measurements are not only essential to unraveling the aerodynamics and flight control mechanism of cicada flight, but also provide insight for understanding the flight capacity of these insects in connection with their biology and ecology. Another unique aspect of this work is that the flight data used for analysis covers a wide range of flight modes and body orientations. On the contrary, most of the previous studies on animals' free flight focused on a specific flight mode such as hover [Fry et al., 2005], forward flight [Wan et al., 2015], yaw turn [Bergou et al., 2010] or banked turn [Fry et al., 2003]. Therefore the conclusions obtained from those studies cannot be generalized to other flights without further investigations. Finally, the large relative body size combined with the simple and effective flight control strategy observed in this work, provides a guideline for the design of Micro Air Vehicles (MAV).



Figure 4.1: Cicada in free flight. A selected sequence of high speed images of cicada in forward flight are shown. It is evident that the wing deforms significantly specially during upstroke.

\subsection{Tracking and measurement of cicada motion in free flight}

Five cicadas (Tibicen linnei) were captured in Dayton, Ohio. After recording several flights of each individual, the wing and body length ( $35 \pm 2 \mathrm{~mm}$ and $30 \pm 1 \mathrm{~mm}$ for wing and body, respectively) as well as the body mass $(1050 \pm 100 \mathrm{mg})$ were measured. The recording area is equipped with three 
orthogonally placed Photron Fastcam SA3 60k high speed cameras synchronized to record at 1000 frames per second. A 3D surface reconstruction technique is then applied to the output from the cameras [Koehler et al., 2012]. Although the body of the cicada stays relatively rigid, the wings deform when flapping (Figure 4.1). To obtain rigid wing kinematics, root mean square plane of the wing is defined based on the position of the marker points on the wing at each frame. The orientation of the rigid wing relative to the body was then expressed by three Euler angles; flapping, deviation and pitch. The flapping angle represents the forward-backward motion of the wing. Deviation is up and downs motion of the wing blade with respect to its joint and pitch is the wing rotation about its hinge axis to the body (Fig). The stroke plane angle is defined by Equation 4.1

$$
\beta=\tan ^{-1}\left(\frac{\dot{\phi}}{\dot{\theta}}\right)
$$

where $\dot{\phi}$ and $\dot{\theta}$ are the flapping and deviation velocities of the wing.

\subsection{Quasi-steady calculation of aerodynamic force}

The aerodynamics force is calculated using the quasi-steady model presented in [Andersen et al., 2005]. The accuracy of the aerodynamic force prediction was examined by comparing the predicted force with computational results on cicada forward flight in [Wan et al., 2015] (Figure 4.2).

\subsection{Body kinematics of cicada free flight}

A total of 42 wingbeats composing of flights with both small and large body reorientations was reconstructed. A selected sequence of images from the reconstructed wing and body are shown in figure $1 \mathrm{~b}$ with the quantitative measurements of the body displacement and orientation being presented in Figure 4.3. Flight 1, consists of two phases of moving on a straight line with a small body pitch angle and an average forward velocity of $1.88 \mathrm{~m} / \mathrm{s}$ followed by pitching up to 45 deg. Flight 2 is a banked-turn during which the flight heading changed by 150 deg. The body rolled to the left within the first two flapping stokes, reaching a 90 deg bank angle. The maximum body roll velocity approached $4000 \mathrm{deg} / \mathrm{s}$ in this phase. The turn is followed by a slow roll back and flying forward while maintaining the body orientation. Flight 3 resembles an Immelmann turn which 


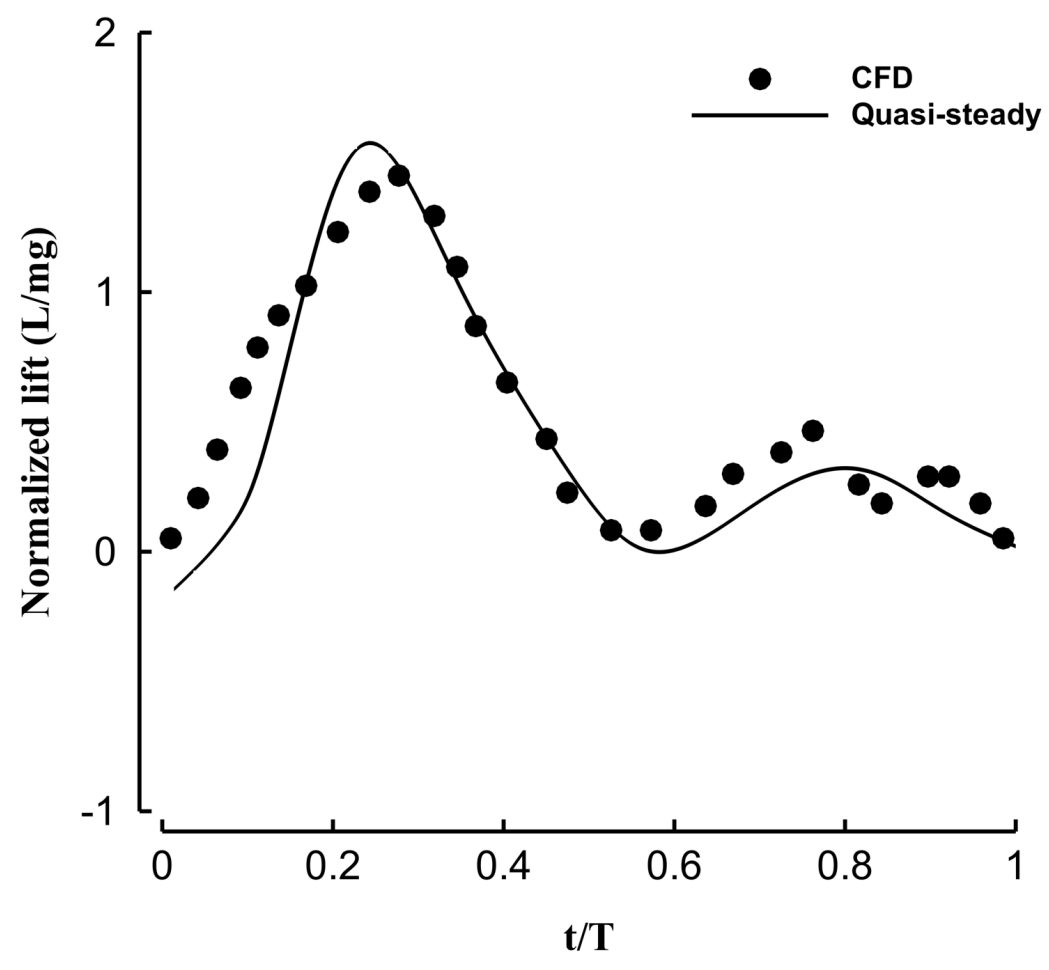

Figure 4.2: Validation of quasi-steady force calculations in cicada. Validation of the quasisteady model with high fidelity simulations of cicada in forward flight in [Wan et al., 2015]. 
consists of an ascending half-loop followed by a fast roll. After takeoff and a short phase of forward flight, the cicada pitched up in a vertical loop until it attained an upside down orientation with respect to the ground. The maximum pitch velocity exceeded $3000 \mathrm{deg} / \mathrm{s}$ and was reached at the early stages of pitching up phase. Subsequently, the cicada rolled to reposition the body in straight flight orientation. The radius of turn in this flight was only 0.9 body lengths. In flight 4 , the cicada body pitched up from 0 to $90 \mathrm{deg}$ within two wingbeats and continued to ascend while maintaining its orientation (body axis normal to the ground) for the next five wingbeats. The vertical velocity of the center of mass was $0.36 \mathrm{~m} / \mathrm{s}$ during this phase. The initial phase was followed by a fast spinning which altered the body's bank angle more than $180 \mathrm{deg}$. Rotations faster than $700 \mathrm{deg} / \mathrm{s}$ occurred about an axis which lies in the body's frontal plane with the angle between rotation axis and the body normal being $92 \pm 22 \mathrm{deg}$. Several performance parameters of these flights are summarized in Table 4.2.

\begin{tabular}{cccccccc}
$\begin{array}{c}\text { Total time } \\
\text { (S, WB) }\end{array}$ & $\begin{array}{c}\text { Avg. hor. } \\
\text { velocity } \\
(\mathbf{m} / \mathbf{s}, \mathbf{B L} / \mathbf{W B})\end{array}$ & $\begin{array}{c}\text { Avg. ver. } \\
\text { velocity } \\
(\mathbf{m} / \mathbf{s}, \mathbf{B L} / \mathbf{W B})\end{array}$ & $\begin{array}{c}\text { Heading } \\
\text { change } \\
(\mathbf{d e g})\end{array}$ & $\begin{array}{c}\text { Avg. pitch } \\
\text { velocity } \\
(\mathbf{d e g} / \mathbf{s}, \mathbf{d e g} / \mathbf{W B})\end{array}$ & $\begin{array}{c}\text { Avg. roll } \\
\text { velocity } \\
\text { (deg/s, deg/WB) }\end{array}$ & $\begin{array}{c}\text { Turn } \\
\text { radius } \\
\text { (BL) }\end{array}$ \\
\hline Flight 1 & $0.15,7$ & $1.88,1.35$ & $-0.51,-0.37$ & 10 & 500,11 & - & - \\
Flight 2 & $0.14,7.5$ & $0.83,0.55$ & $0.15,0.09$ & 150 & - & 2600,42 & 2.5 \\
Flight 3 & $0.21,12.5$ & $0.29,0.16$ & $0.38,0.21$ & 170 & 2800,47 & - & 0.9 \\
Flight 4 & $0.32,15$ & $0.48,0.24$ & $0.36,0.26$ & 10 & 1800,38 & 1300,31 & 0.9
\end{tabular}

Table 4.2: Performance parameters of different cicada flights. BL and WB stand for Body Length and Wing Beat, respectively.

\subsection{Wing kinematics}

Despite the large body reorientation, the motion of the wing relative to the body was stereotypical among all the flights. The wing tip trajectories were oval shaped with the ratio of the minor to major axis lengths of $0.28 \pm .09$ (Figure 4.4). The angle between the body and the stroke plane remains relatively invariant; $48.2 \pm 10.2 \mathrm{deg}(\mathrm{n}=84)$ and $45.9 \pm 13.8 \mathrm{deg}$ for downstroke and upstroke respectively. Besides slight variations in the wing stroke plane, the flapping amplitude of the wing 


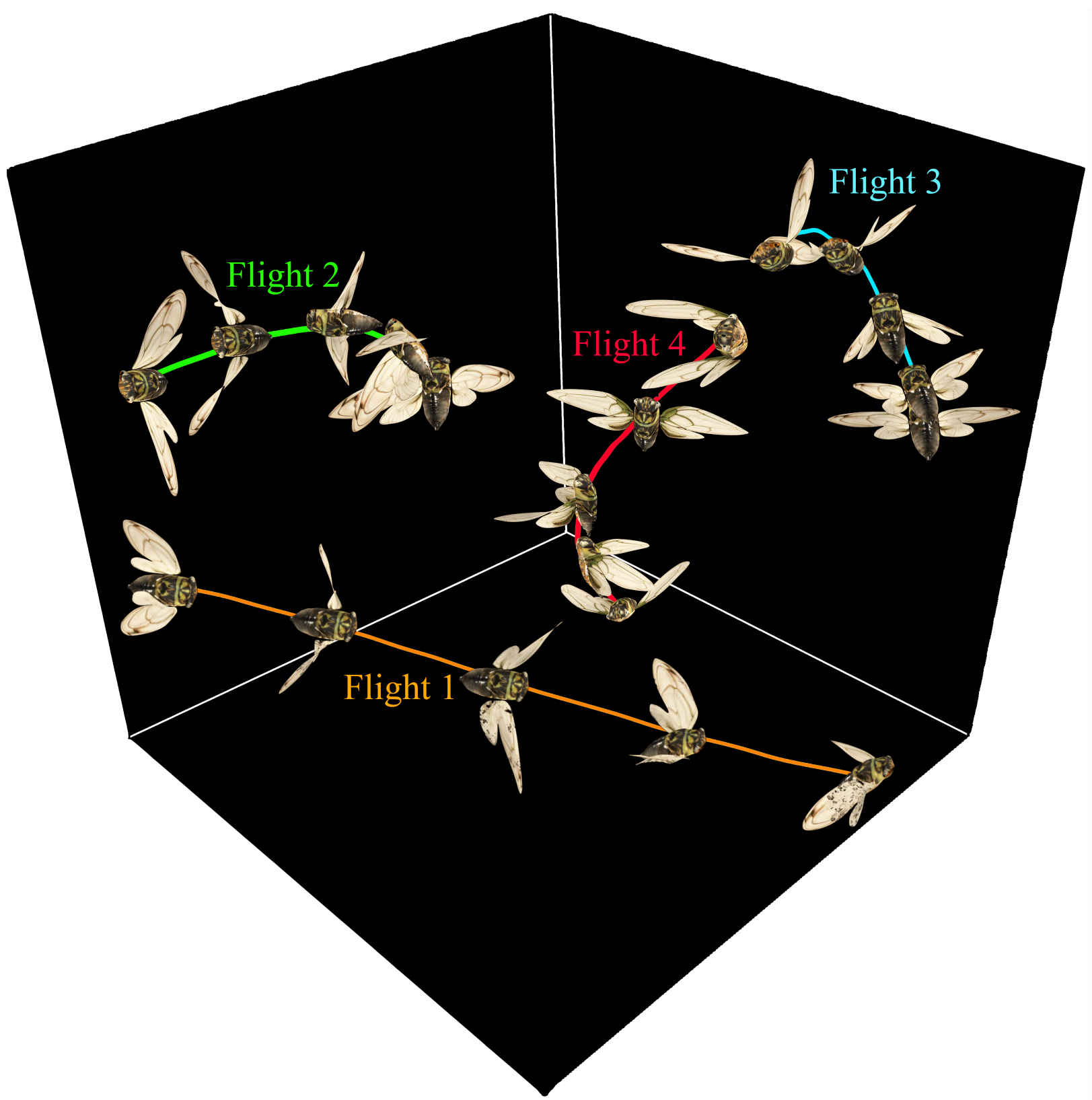

Figure 4.3: Reconstructed wing and body of cicada in free flight. A sequence of images of the reconstructed wing and the body of four cicada in free flight are shown. The trjectory of the center of mass for each flight is indicated by a distinct color. 
in the stroke plane as well as the mean flapping angle (average location of the wing with respect to the center of mass) was modulated from one stroke to another. The flapping frequency varies slightly among the individuals as well as during the maneuver; $50.5 \pm 8.8 \mathrm{~Hz}(\mathrm{n}=42)$. In addition, cicadas were able to adjust the ratio of the downstroke to upstroke duration between 0.6 and 1.4. Orientation of the wing in the stroke plane was adjusted via modulating the wing pitch angle. The pitch angle of the wing is shallow in downstroke and varies largely; $-7.1 \pm 11.5 \mathrm{deg}$. This angle is higher in upstroke; $31.6 \pm 20.1 \mathrm{deg}$. Detailed measurement on the wing kinematics can be found in Figures 4.5, 4.6, 4.7 and 4.8.

A

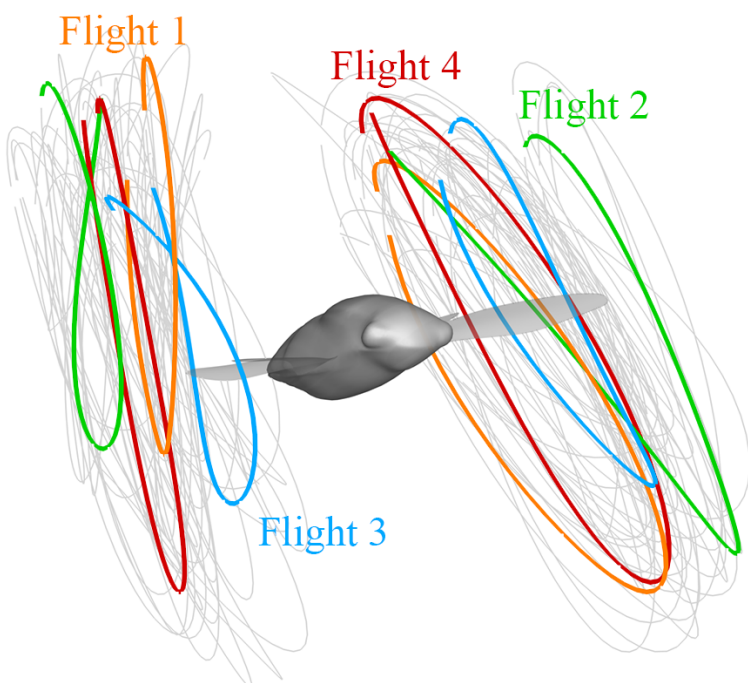

B

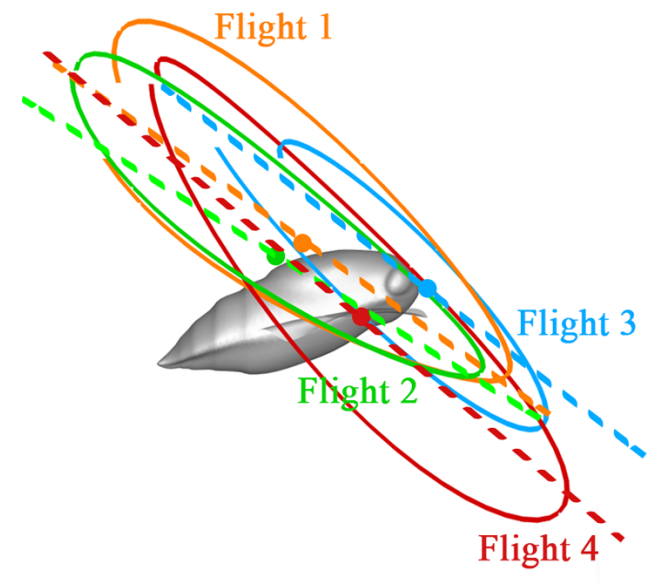

Figure 4.4: Wing tip trajectory in cicada free flight. (A) The wing tip trajectory of all recorded wingbeats are shown in gray. Atypical trajectory from each flight is highlighted by a color. The wing moves on an oval trajectory. (B) Side view of the typical wing tip trajectory shows that the stroke plane angle remains invariant regardless of the body orientation (dashed straight lines). Yet the center of the oval (which is shown by a closed circle with the same color as the trajectory curve) as well as the amplitude of flapping varies among flights and within one flight.

\subsection{Investigation of the flexibility of aerodynamic force direction}

To interpret the dynamics of these flight, we calculated the aerodynamic force generated by the wings, using a quasi-steady model. These forces are then summed up and averaged over each downstroke and upstroke. The vector of the half-stroke-averaged aerodynamic force and normalized by the body weight. This vector as well as the normal vector to the body were then superimposed 
Flight 1



Figure 4.5: Wing and body kinematics of cicada in Flight 1. 
Flight 2



Figure 4.6: Wing and body kinematics of cicada in Flight 2. 
Flight 3



Figure 4.7: Wing and body kinematics of cicada in Flight 3. 
Flight 4



Figure 4.8: Wing and body kinematics of cicada in Flight 4. 
on the images of the reconstructed wing and the body motion for Flight 1 in Figure 4.9. The average force in downstroke and upstroke are shown with different colors for clarity. Aerodynamic force is generated during both upstroke and downstroke. Yet, there was a distinct difference between the two. Visual inspection of Figure 4.9, demonstrates that the downstroke force sustains a relatively normal direction with respect to the body. In a forward flight, downstroke force will generate the lift force required to keep the insect aloft. Force in upstroke points forward and downward making a shallow angle with the flight path. These results are consistent with the previous studies on the aerodynamics of cicada forward flight [Wan et al., 2015]. Several insects and birds use a similar strategy; generating lift in downstroke and thrust in upstroke.

To quantify the alternation of the aerodynamic force direction during flight, we calculated the angle between the averaged aerodynamic force in downstroke, , and upstroke, , and the normal vector to the body for all 42 wingbeats. The results are plotted in Figure 4.10. Visual inspection of this figure illustrates that the orientation of the aerodynamic force in both downstroke and upstroke is restricted relative to the body, regardless of the body orientation. In particular, the downstroke force maintains a uniform orientation with respect to the body's normal vector, with the angle between the two vectors being $17 \pm 7 \mathrm{deg}$. The angle between the upstroke force and the body normal is $135 \pm 10 \mathrm{deg}$.

\subsection{Control of the force direction and magnitude.}

Small wing pitch angle during downstrokes, causes the downstroke force to maintain a relatively normal orientation relative to the body. In upstroke, wings are pitched up relative to the body which directs the force at a slight angle relative to the stroke plane. By altering the wing pitch angle with respect to the body (and therefore to the stroke plane), cicada alters the aerodynamic force direction. The orientation of the aerodynamic force relative to the body normal was strongly correlated with the angle between the wing and the stroke plane; $R^{2}=0.32$ and $R^{2}=0.79$ for downstroke and upstroke, respectively. Yet, modulating the wing pitch angle provides more control over the wing orientation in a vertical plane to the body. However, our analysis shows that cicadas are capable of generating bilaterally asymmetric aerodynamic force without banking the body to redirect the force, as was found to be used by smaller insects. This was achieved by changing 




Figure 4.9: Aerodynamic force direction relative to the body in cicada cruise. Aerodynamic force vector maintains a uniform orientation with respect to the body. The half-stroke-averaged aerodynamic force vector is superimposed on the reconstructed wing and body for flight 1 . The distance between the images in the flight sequence was increased to reduce overlapping. The average force in downstroke and upstroke are shown by green and blue arrows respectively. The normal unit vector to the body is shown by a filled-headed black arrow. The length of the arrows represents their relative magnitude with the aerodynamic force being normalized by body weight. 
A

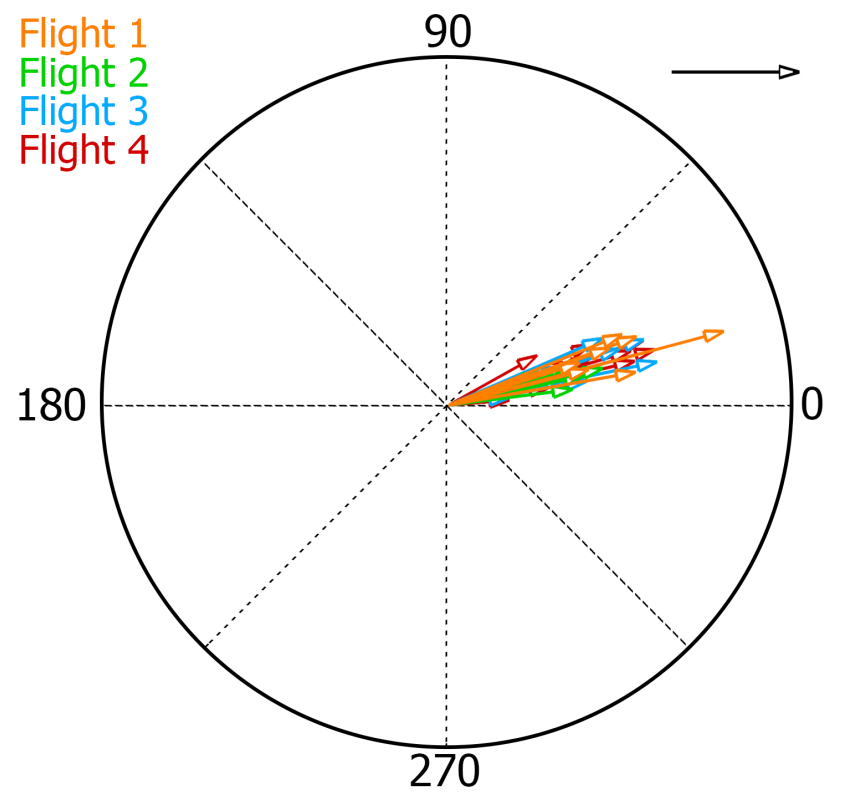

B



Figure 4.10: Orientation of the aerodynamic force relative to the body normal in cicada free flight. The orientations of downstroke (A) and upstroke (B) force with respect to the normal to the body are colored differently for each flight. The length of the arrow represents the magnitude of the normalized force. The clustering of the arrows shows that the orientation of the aerodynamic force relative to the body is independent of the body orientation. 
the wing trajectory, altering the flapping and deviation angle relative to the body. The lateral direction of the force is mainly modified during upstrokes. For instance in 3th upstroke in flight 3, the upstroke force is tilted to the right by the extending the flapping and deviation rotations of the right wing further forward and downward, respectively.

Cicadas were capable of altering the force magnitude. Overall, the magnitude of the force in upstroke as well as its variations were larger than those in downstroke; $0.60 \pm 0.25$ and $0.78 \pm 0.28$. To alter the force magnitude, cicada changes the wing velocity by adjusting the stroke amplitude and flapping frequency. The magnitude of the force was found to be strongly correlated with the square of the wing velocity $\left(R^{2}=0.45\right)$.

\subsection{Change of flight path}

To investigate the mechanism by which cicadas redirect the force in the global frame, we examined the relative orientation of the rotation axis (for rotations faster than $700 \mathrm{deg} / \mathrm{s}$ ) and the half-strokeaveraged force. Body rotations that occur about the force vector preserve the orientation of the force in the global frame and do not result in flight trajectory changes. On the contrary, rotations about an axis that deviates from aerodynamic force, redirects the force and thus alters the flight trajectory. Our results show that body rotations occur about an axis which predominantly redirects the aerodynamic force; the angles between the rotation axis and the downstroke and upstroke forces were $75 \pm 12 \mathrm{deg}$ and $56 \pm 21 \mathrm{deg}$, respectively.

Despite the directional limitation of the aerodynamic force in the body frame, cicadas can generate rotations about the body roll and pitch axes. Roll torque was produced by asymmetrically varying the angle of attack of the bilateral wings. The magnitude of the roll torque was strongly correlated with the wing angle of attack $\left(R^{2}=0.6, \mathrm{n}=84\right)$. Pitch torque was exerted by shifting the mean position of the wing to offset the force relative to the center of mass (Figure 4.11), similar to the technique employed by fruit flies [Zanker, 1988]. The magnitude of the pitch torque was directly correlated with the average wing deviation angle $\left(R^{2}=0.63\right)$. Since, the downstroke force is normal to the cicada body, no significant yaw torque can be generated during this half of the flapping cycle. Our measurements show that the magnitude of the yaw velocity is smaller than that of pitch or roll velocity and oscillates within wingbeats, increasing in upstroke and decreasing in downstroke. The 


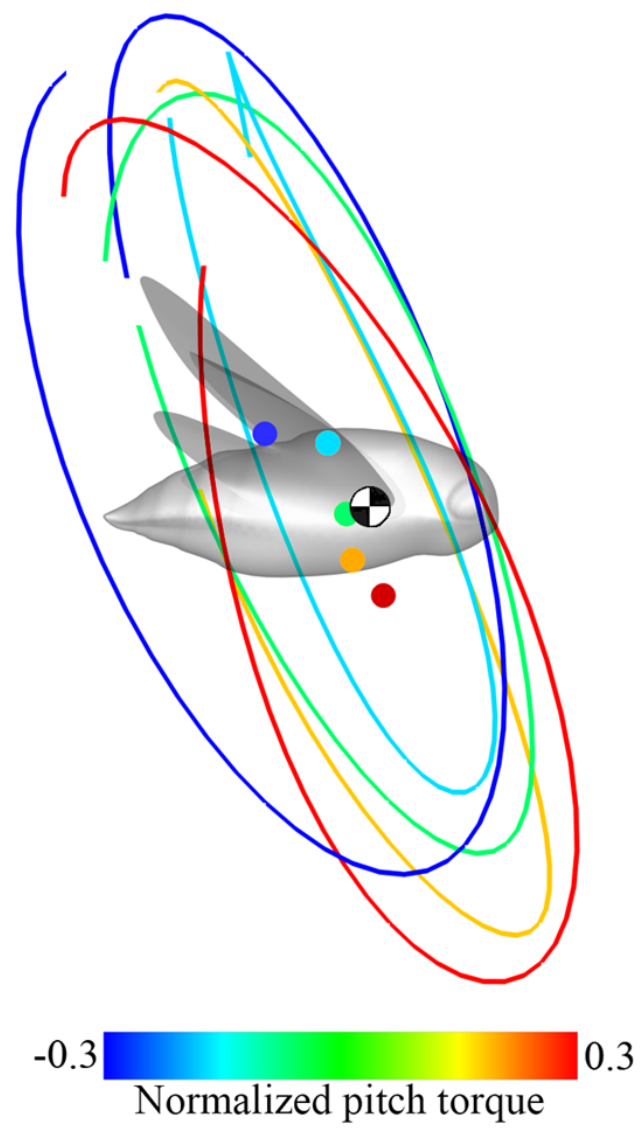

Figure 4.11: Effect of wing trajectory on aerodynamic pitch torque in cicada flight. Pitchtorque-producing wing tip trajectories of flight 4 are colored by the stroke averaged pitch torque generated by the wing. The cicada shifts the wing's average position (shown by a closed circle with the same color as the tip trajectory) relative to the center of mass (black and white circle) to generate pitch torque. 
decrease in the yaw velocity in downstroke is due to a strong passive damping caused by the body rotation [Dillon and Dudley, 2004].

\subsection{Discussion}

Animals living in different environment in their life time face tradeoffs between maximizing functionality in different settings [Fry et al., 2003, Gotz and Wehrhahn, 1984]. Cicadas spend several years of their lives under the soil and emerge to the top after they mature. They are known for their long development period as well as their large body sizes which is one of the largest among Homopteran [Fry et al., 2005]. Body mass is known to affect the flight performance with larger flyers having narrower flight capabilities [Ellington, 1991]. In spite of that, our observations show cicadas are capable of executing fast aerial maneuvers. In the voluntary turns recorded in this experiment, the average speed of the heading change was about 1000-1500 deg/s which is at about the same range as that of other insects such as flies [Ellington, 1984b], dragonflies [Alexander, 1986] and damselflies (authors measurements). While the time duration of the voluntary aerial maneuvers of cicada were found to be similar to those of other insects, the brevity of these flights in wingbeat time should not be underscored. For instance in Flight 4, the roll velocity of the body increased $3000 \mathrm{deg} / \mathrm{s}$ within only one wingbeat which requires generation of large rolling torque during only one downstroke and one upstroke. Therefore, when the flight capabilities are analyzed in wingbeat time, low flapping frequency fliers such as cicada, dragonflies and damselflies have superior capabilities when compared to high flapping frequency insects such as flies.

To investigate the aerodynamic basis of cicada aerial maneuvers, we examined the coordination between the motions of the wings and body in connection with the aerodynamic force production. Our results show that downstroke and upstroke have distinct roles in flight dynamics of cicada. When flying on a straight line, the downstroke carries out the entire weight supporting role while upstroke provides the propulsive force. Yet, these roles are interchangeable when body reorients during aerial maneuvers. For instance in the turning flights reported in this work, the downstroke force provided the centrifugal force required for bending the flight path while the weight supporting role was mostly carried out by upstroke force either due to the rotation of the wing stroke plane with the body (in Immelmann flight) or the lateral redirection of the upstroke force (in banked turn). 
The directional limitation of the aerodynamic force has other implications. The vertical orientation of the downstroke force reduces energy expenditure by evading generation of drag and employing all the aerodynamic force (generated in downstroke) to support the body weight. The orientation of the upstroke force eliminates the need for tilting the stroke plane angle to propel the flight. Since wing stroke plane is fixed to the body, redirecting the wing stroke plane requires reorientation of the whole body. Because of the large moment of inertia of the cicada body, this would substantially compromise the agility of the flight when horizontal acceleration is needed [Ellington, 1999]. On the other hand, agility in deceleration is usually not as essential as it is in acceleration. To decelerate, i.e. for landing for instance, cicadas reorient the wing stroke plane by pitching the body up which subsequently decreases the propulsive component of the upstroke force and generates backward component in downstroke force. It also increases body drag by exposing a larger area to the flow current which dampens the velocity as well. An example of this phenomenon is the second phase of flight 1 during which the cicada pitches up and slows down as it approaches the wall in the small shooting area. This strategy works well considering cicada's life style which includes frequent landings on the tree trunks. Yet, the distinction between the downstroke and upstroke force directions sacrifices the body weight supporting role of the upstroke. To compensate, the sharp angle of the stroke plane relative to the body provides large angle of attack even at small wing pitch angles which helps generating high force magnitudes.

In addition, having an aerodynamic force fixed to the body could potentially compromise the maneuverability. For instance, the cicadas executed large fight heading changes by a banked or Immelmann turn rather than a yaw turn, which was found to be widely used by other insects [Altshuler et al., 2005, Ellington, 1984b, Dillon and Dudley, 2004, David, 1978] and some birds such as hummingbirds [Dillon and Dudley, 2004]. In a pure yaw turn, a flying animal generates torque only in one direction which eliminates the task of changing the flight path. We cannot exclude the possibility that cicadas are limited by their biomechanical constraints for execution of a yaw turn. Since the downstroke force is normal to the cicada body, no significant yaw torque can be generated during this half of the wing stroke. Our measurements show that the magnitude of the yaw velocity is smaller than pitch or roll and oscillates within wingbeats, increasing in upstroke and decreasing in downstroke. The decrease in the yaw velocity in downstroke is due to a strong passive damping caused by the body rotation [Dillon and Dudley, 2004]. 


\subsection{Conclusions}

We conclude that all the free flights studied here are governed by a unified force control strategy, despite the fact that they share little in common with regard to the body orientation and motion. The restricted variations in the orientation of the aerodynamic force relative to the body necessitates the body to reorient for redirecting the force and changing the flight path. Directional limitation of the aerodynamic force in the body frame also simplifies the mechanics of the wing hinge, as it reduces the need for implementing complex alternations in the wing motion. The importance of these results is twofold; they clarify the aerodynamics and mechanics of cicada free flight, and they prove that force vectoring can be successfully implemented for designing large payload and yet maneuverable flapping wing Micro Air Vehicles (MAVs).

\subsection{Acknowledgement}

The 3D surface reconstruction of the wings and the body is performed by Nidhin Babu. He also made Figure 4.3 and 4.5 . 


\section{Chapter 5}

\section{Damselfly yaw turn}

\subsection{Introduction}

Maneuverability provides insects with the ability to evade predators, or capture prey and therefore plays a vital role in their survival and evolution [Dudley, 2002]. The study of maneuvering flight of insects has led to extensive advancements in our understanding of aerodynamics [Lehmann and Dickinson, 2001, Muijres et al., 2014], flight dynamics and control of flapping flight [Hedrick et al., 2009, Heide and Gotz, 1996], neuro-muscular control of the wing motion [Balint and Dickinson, 200 Lehmann and Dickinson, 1998, Trimarchi and Schneiderman, 1995], and sensory mechanisms of insects [Bender and Dickinson, 2006, Dickinson et al., 1999, Muijres et al., 2014, Sherman and Dickinson, 2003, Sherman and Dickinson, 2004]. However, while several studies focusing on the maneuvering flight of insects such as flies [Bergou et al., 2010, Balint and Dickinson, 2004, Fry et al., 2003], blowflies , [Balint and Dickinson, 2001, Balint and Dickinson, 2004], honeybee [Bastian, 1972], hawk moth [Hedrick and Daniel, 2006], and dragonflies [Alexander, 1986, Wang et al., 2003] have surfaced in the scientific community in recent decades, little attention has been paid to the flight of damselflies. Damselflies are aerial predators and their survival hinges on their superiority over other insects in maneuverability and agility. They are capable of hovering, flying backward, darting abruptly during flight [Cobb, 2010] and executing other types of aerial maneuvers.

Few previous studies of damselflies indicate that they are capable of accurately modulating their wing flapping amplitude, flapping frequency, inclination of the stroke plane with respect to the flight path, downstroke/upstroke ratio and phase difference between the forewings and hindwings 
to control body motion through the air [Ruppell, 1989, Wakeling and Ellington, 1997]. Yet, these studies lack a detailed report on the kinematics as well as the aerodynamics of flight. Current advances in high speed photogrammetry techniques enabled us to gather accurate flight data, and carefully analyze the details of the wing and body kinematics to reveal the mechanisms by which damselflies achieve high levels of aerial maneuverability. In this study, we recorded yaw turns of three species of damselflies with distinct wing and body morphologies. Despite the similarities observed in the body kinematics, different species employed their wings in distinct ways to achieve the same performance. Nevertheless, the coordination between the wings was similar. The mechanism by which damselflies steer a yaw turn were found to be similar in some aspects to what was observed in other insects such as flies. However, several unique features were found in the regulation of the wing motion and the aerodynamic yaw torque generation which was not previously reported in flight maneuvers of other insects.
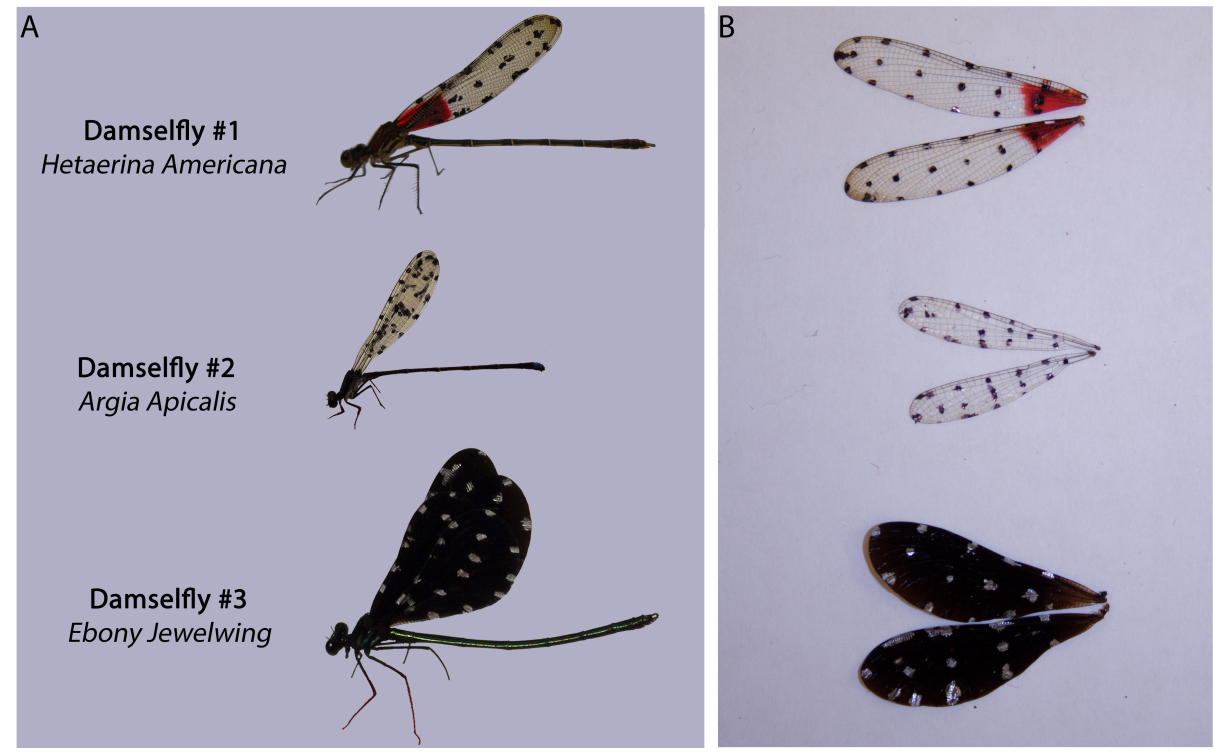

Figure 5.1: Three species of damselfly. (A) A representative of each one of the three species of damselfly in this experiment are shown side by side for comparison. The wings are artificially dotted for the tracking purpose (refer to methods section for details). Species name is shown next to the corresponding picture. (B) An image of a left fore and hind wing of each species is shown. The dots are the marker points placed on the wing for tracking the wing motion.

In order to execute a fast maneuver, an insect finely tunes its wing kinematics on a stroke by stroke basis. Generally, flying insects use a variety of different kinematic alterations to perform aerial 
maneuvers such as changes in the wing stroke amplitude [Lehmann and Dickinson, 1998], wing pitch angle [Bergou et al., 2010], speed and timing of wing rotation [Alexander, 1986, Dickinson et al., 1993], etc. Small insects such as fruit flies and drone flies navigate a yaw turn mainly by regulating the wing pitch angle which results in changes in the angle of attack of the bilateral wings [Zhang and Sun, 2010, Bergou et al., 2010]. Likewise, incremental increases in the outer wing flapping amplitude as well as the stroke plane angle were also found to contribute to the generation of the yaw torque [Fry et al., 2003]. Damselflies differ significantly from these insects; fruit fly and drone flies, both in wing and body morphology as well as flapping frequency. Furthermore, damselflies have very slender bodies and four independent wings. It is not obvious how these differences affect their wing and body kinematics as well as flight dynamics. A complete understanding of this problem requires in-depth research on different aspects of the flight as well as the physiology of these insects and is beyond the scope of this paper. Our aim is to report the data of our observation of damselfly yaw turns and to explain the mechanisms employed by damselflies to execute this type of maneuver.

\subsection{Damselflies; Body and wing morphology}

Different species of damselflies (Hetaerina Americana, Argia Apicalis and Ebony Jewelwing) were caught in the wild during the months of July and August, 2010 and 2011 in Dayton, Ohio. Prior to the experiments, the caught damselflies were kept in ventilated containers for no more than an hour at outdoor temperatures $(70-80 \mathrm{~F})$ in order to prevent inactivity during the time of experiments. Initiation of flight during the experiments was not forced or stimulated by any external means such as tail prodding; rather, the damselflies initiated flight autonomously. After recording the flight, the wings were removed and the mass and length of the wings and the body were accurately measured.

Free flight of 15 damselflies of three different species, Hetaerina Americana, Argia Apicalis and Ebony Jewelwing, were recorded. Images of a representative insect of each species are shown side by side in Figure 5.1 for comparison. Table 1 summarizes the morphological parameters of the wings and the body as well as the sample size for each species. Overall body shape was similar among species, characterized by a wide head, a relatively small thorax and an elongated abdomen. The abdomen length was about $80-82 \%$ of the total body length in all species. Average body mass varied 
significantly among the species but the body length was found to be strongly correlated with the body mass (Figure 5.2).


Figure 5.2: Morphology of three damselfly species. Body length was found to be strongly correlated to the body mass (A). In addition, the length of the wing increases directly with the length of the body (B). The actual area of the wing is strongly correlated with the product of the wing length and the maximum wing chord for both fore and hindwings.

Figure 5.1 shows a picture of the left forewings of a representative damselfly of each species. As evident form the graphic, the size and shape of the wings varies across species. Wing length was significantly correlated with the body length (Fig. 2B) with the length of the wing being about $63 \%$ of the body length. The wing aspect ratio was found to be independent of the body morphology. The wing area was strongly correlated with the product of the wing length and maximum wing chord. This area is referred to as rectangular area of the wing in Fig. 2C. The actual area of the wing was accurately calculated using an image of the wing (refer to the methods section). One can obtain an accurate wing area estimation using only the wing length and chord measurement and employing the analytical relationship given in Figure 5.2.

\subsection{Radius of turn}

The radius of turn is one of the parameters which is conventionally used to characterize the maneuverability of a flying vehicle. In insect flight, this value is equivalently important showing how much distance an insect needs to travel to perform a maneuver. Conventionally the smaller the radius of turn the more maneuverable a flying object is. A common way of calculating turn radius 
such as that described in (Jantzen and Eisner, 2008) depicts a point moving around a 2D curve. This definition is based on the ratio of the translational velocity of the center $f$ mass. However, the shortcoming of this approach is that there is no consideration of any changes in turn radius due to angular rotations about the body axes and thus the radius of curvature is over-estimated. In this work, we used an alternative definition for the radius turn defined by Equation 5.1.

$$
r=\frac{|U|}{\left|\Omega_{\text {yaw }}\right|}
$$

$\left|\Omega_{\text {yaw }}\right|$ and $|U|$ are the magnitudes of the body yaw and translational (planar) velocities. Based on this definition, the smaller the ratio of the translational to rotational velocity the smaller the radius of turn is. The values reported are the average radius of turn throughout the maneuver.

\subsection{Wing area calculation}

Damselfly wings come in different sizes and shapes depending on the species. For each damselfly in this study, pictures of the wings were taken and the corresponding virtual wings were reconstructed in Autodesk MAYA as shown in Figure 5.3. Using the surface area function polyEvaluate-area, the area of each individual mesh can be calculated and added up around the perimeter of the image to calculate the total surface area of the wing. This non-dimensional area is dimensionalized based on the non-dimensional span and chord as well as the dimensional span and chord measured from the real insect as shown in Equation 5.2

$$
A=\hat{A} \frac{R c}{\hat{R} \hat{c}}
$$

\subsection{Quasi-steady estimation of the aerodynamic yaw torque}

The aerodynamic force and torque are estimated using a quasi-steady model proposed by [Pesavento and Wang, 200 Using blade element theory and assuming no interactions between the $2 \mathrm{D}$ blades of the wing, the total aerodynamic force and torque on the wing can be calculated by integrating across the wing length. The yaw torque on a thin 2D blade is calculated by Equation 5.3. 


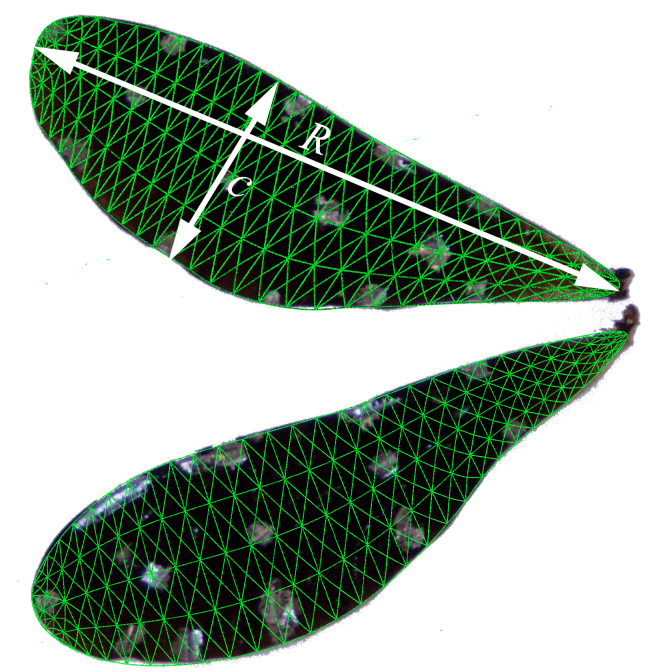

Figure 5.3: Damselfly wing model used for area measurements. The model is based on the real image of the wing. The model is made in Autodesk MAYA. The wing length and maximum chord are shown on the figure.

$$
d \tau_{z}^{\prime}=r_{i} d F_{y}^{\prime}
$$

$C_{r}, C_{T}, C_{D_{0}}$ and $C_{D_{\pi / 2}}$ constants were set to $\pi / 2,1.8,0.21$ and 3.35 , respectively. The angle of attack of the wing, $\alpha$, is defined by Equation 5.4 (Fig. 10B).

$$
\alpha=\tan ^{-1}\left(v_{z}^{\prime} / v_{y}^{\prime}\right)
$$

$v_{y}^{\prime}$ and $v_{x}^{\prime}$ are the velocity components of the wing parallel and normal to the wing chordline (Figure5.4). The angular velocity of the wing in the wing-fixed coordinate system, $\omega^{\prime}=\left(\omega_{x}^{\prime}, \omega_{y}^{\prime}, \omega_{z}^{\prime}\right)$ is calculated by Equation 5.5

$$
\begin{aligned}
& \omega_{x}^{\prime}=\omega_{x}-\omega_{z} \sin \theta \\
& \omega^{\prime}{ }_{y}=-\omega_{z} \cos \theta \cos \eta+\omega_{y} \sin \eta \\
& \omega_{z}^{\prime}=\omega_{z} \cos \theta \sin \eta+\omega_{y} \cos \eta
\end{aligned}
$$

$\omega=\left(\omega_{x}, \omega_{y}, \omega_{z}\right)$ is the absolute angular velocity of the wing expressed in the body-fixed coordinate system (Figure5.4). The net angular velocity of the wing is the sum of the wing velocity with 
A

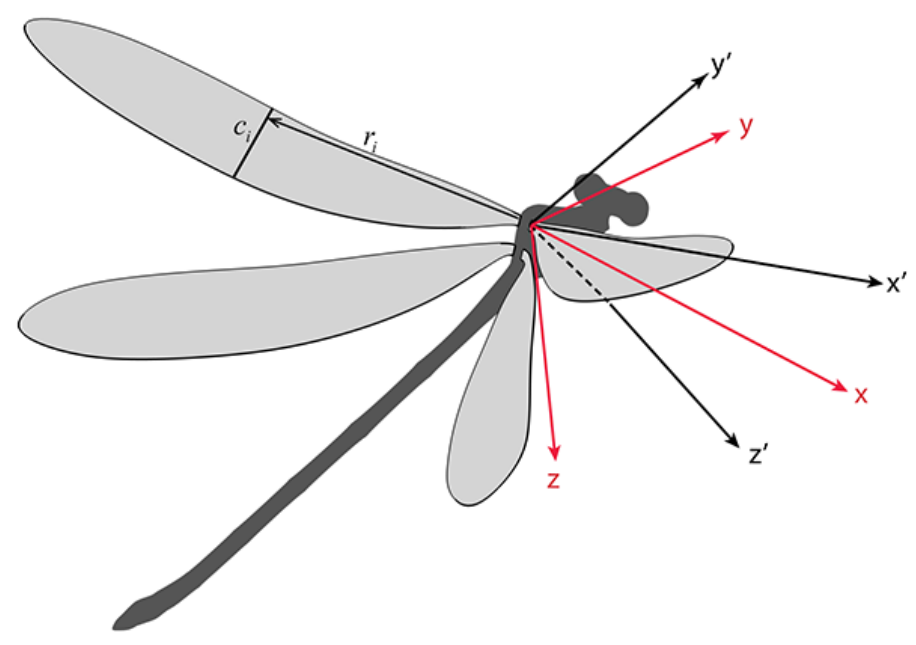

B

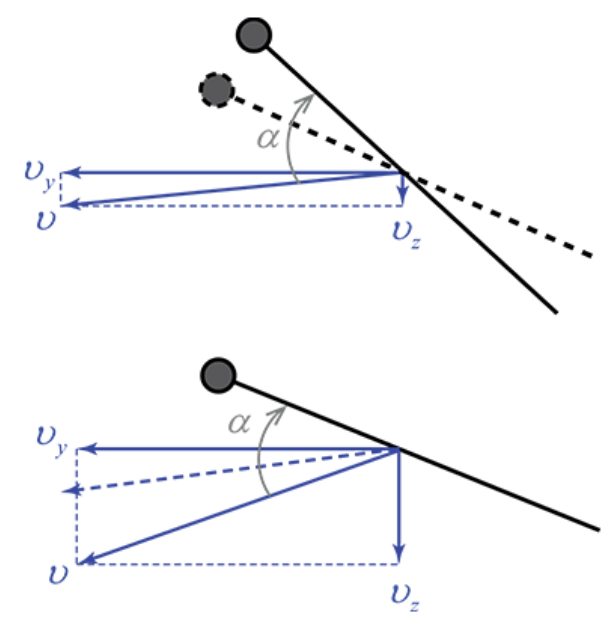

Figure 5.4: Definition of the wing and body coordinate systems. $x^{\prime} y^{\prime} z^{\prime}$ coordinate system is fixed on the wing and moves with it. xyz-axes is fixed to the body. (B) Angle of attack of the wing is the angle between the wing chordline and direction of incoming velocity. Leading edge of the wing is shown by a closed circle. This angle can be altered by either pitching the wing or changing the direction of incoming velocity to the wing.

respect to the body and the body velocity with respect to the inertial frame of reference (Equation $5.6)$.

$$
\begin{aligned}
& \omega_{x}=\dot{\eta}-Q \\
& \omega_{y}=\dot{\theta}+P \\
& \omega_{z}=\dot{\phi}+R
\end{aligned}
$$

Where $\mathrm{P}, \mathrm{Q}$ and $\mathrm{R}$ are the body roll, pitch and yaw velocities expressed in the body-fixed coordinate system, respectively about the body axes pointing forward, to the right and downward. The magnitude of the translational velocity on each 2D blade of the wing increases by the distance of the blade from the wing root (Equation 5.7).

$$
\begin{aligned}
& v_{y}^{\prime}=r_{i} \omega_{z}^{\prime} \\
& v_{z}^{\prime}=-r_{i} \omega_{y}^{\prime}
\end{aligned}
$$

The calculated force and torque are then transferred to the body-fixed coordinate system using Equation 5.8. 


$$
\begin{aligned}
& F=T F^{\prime} \\
& \tau=T \tau^{\prime}
\end{aligned}
$$

$\mathrm{T}$ is the Euler rotation matrix calculated using the Euler angles of the wing with respect to the body. To test the accuracy of the predicted force and torque, we compared the magnitude of the averaged vertical force to the total vertical acceleration of the each damselfly, $m(a+g)$. Our estimation fell within $10 \%$ of the measured values for all damselflies.

\subsection{Body kinematics in yaw turn}

A total of 30 turning flights were recorded with the magnitude of heading change ranging between 20-180 degrees. Given the variety of the yaw turns recorded, rather than averaging the data for each species we chose a single yaw turn with the largest amplitude and maximum number of wingbeats for analysis. We denote the selected individual from Hetaerina Americana, Argia Apicalis and Ebony Jewelwing as damselfly $\# 1, \# 2$ and $\# 3$, respectively. All selected maneuvers were left turns. Fig. 3A shows the top view, taken by downward facing camera, of a selected sequence of images of the representative turn of a damselfly \#3. This damselfly performed two consecutive turns of 55 and 110 degrees in one trial. The sequence of images shows the larger amplitude turn. The body orientation is shown at the beginning and end of each wing flapping stroke of forewings. The planar displacement of the center of mass was minimal in all recorded turns and consequently resulted in a very small radius of turn (Fig. 3A). The radius of turn, r, was defined as the ratio of the planar translational velocity to the rate of heading change and was only a portion of the body length for all the recorded flights (Table 2). The body kinematics of the representative species during the yaw turn is shown in Fig. 3B. Unlike insects such as flies which maintain high body pitch angle (about $50 \mathrm{deg}$ ) during a yaw turn [Hedrick et al., 2009], the average body pitch angle of the damselflies was low and between 0-15 deg. In most of the recorded flights, variations in the bank and pitch angles are negligible. The minimal variations in the bank and pitch angles together with small turn radii characterize the free flight yaw turns of the damselflies. Table 2 summarizes several parameters that typify the kinematics of these turns. 
A



B

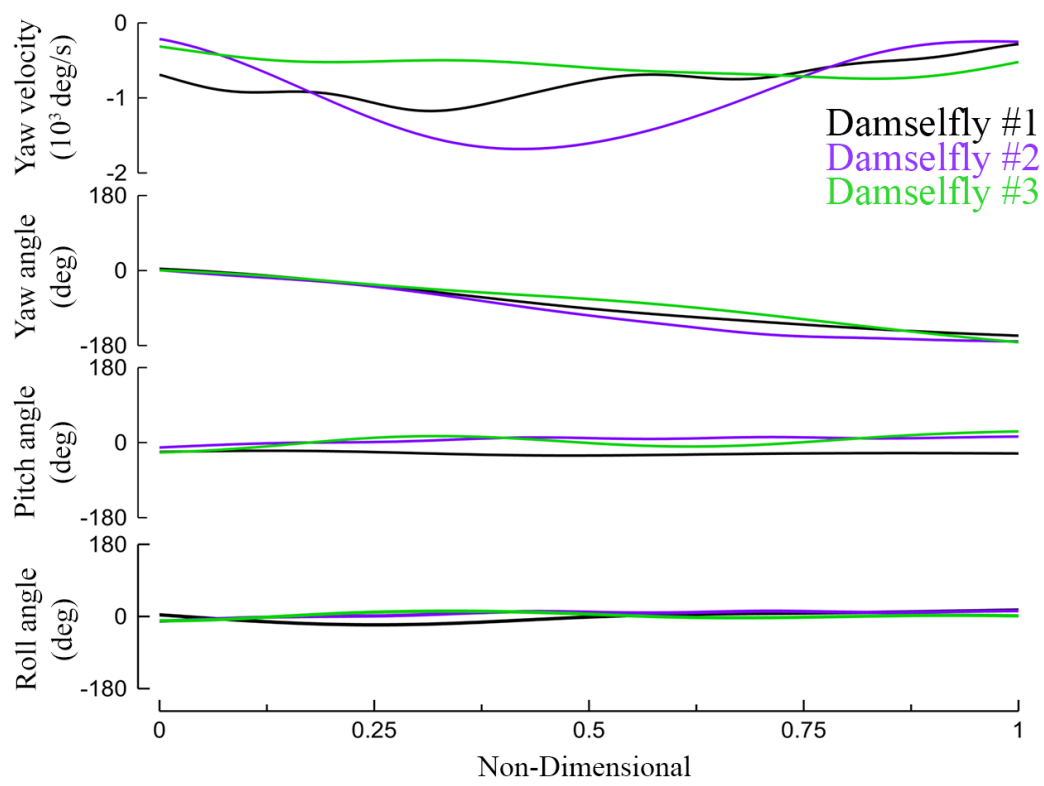

Figure 5.5: Damselfly body motion during an aerial yaw turn. (A) Sequence of images of the yaw turn of damselfly $\# 3$. The selected images were recorded by downward facing camera. The body orientation is shown at the beginning and end of each flapping stroke of the forewings. Radius of turn, $r$, is shown on the figure. (B) Body kinematics of the representative turn of each species is shown versus normalized time which is the ratio of actual time to the maneuver duration. The maximum yaw velocity is indicated by a star symbol. 
All the recorded flights consisted of two stages of acceleration and deceleration, the latter being longer than the former (Figure 5.5). The body yaw velocity rose rapidly during the acceleration phase and attained a maximum value (indicated by a star in Figure 5.5). Only about 30-40\% of the heading change took place during the acceleration phase (Fig. 3A). We did not observe a period of relatively constant angular velocity, as was previously observed in the other flying insects such as fruit flies [Fry et al., 2003]. On the contrary, the acceleration phase was immediately followed by an elongated deceleration phase during which the angular velocity of the body slowly declined. The maximum as well as average yaw velocity varied across the species. However, when calculated in wingbeat time (rather than real time), the average yaw velocity was found to be robust across species for both stages of the maneuver (Table 2) with the body heading angle changing about 30-40 deg per wingbeat. This number is only about $10 \mathrm{deg}$ for flies [Bergou et al., 2010, Fry et al., 2003].

\subsection{Wing kinematics}

Figure 5.6, Figure 5.7 and Figure 5.8 shows the wing kinematics of the representative turns of the three species. Assuming that wing deformations are small, the orientation of the wing can be described by three Euler angles. The wing kinematics is measured with respect to a coordinate system attached to the body (refer to materials and methods section). Flapping motion is defined as the back and forth motion of the wing and is shown as positive when wing is moving forward (downstroke). The up and down rotation of the wing is expressed by the deviation angle and is positive when wing moves up. The wing pitch angle is characterized by the rotation of the wing about its pitching axis close to the leading edge and is defined positive when wing pitches up. The kinematics of fore and hind pairs of the wings are shown with solid and dashed lines, respectively. Red and blue colors indicate the inner and outer wings. All four wings showed significant variation in the kinematics throughout the maneuver. In fact, the asymmetry in the wing kinematics was found to be drastic rather than subtle. This is in contrast to what was observed in maneuvering flight of high flapping frequency insects such as fruit fly and drone fly [Fry et al., 2003, Muijres et al., 2014]. To better illustrate the complexity of the wing motion as well as the extreme asymmetry in the kinematics of all four wings, we showed the wing tip trajectory of damselfly $\# 2$ in Figure 5.9. This damselfly showed the most variability in the wing tip trajectory comparing to other two. The 
trajectories of the fore and hind wings are shown by black and purple lines, respectively. The initial position of the wing tip is indicated by a sphere. Both the perspective and back views are shown for clarity. Figures Figure 5.6, 5.7 and 5.8 and 5 illustrate a potential difference between the mechanisms by which a damselfly steers during an aerial maneuver and that of a high flapping frequency insect such as a fly. In high flapping frequency flight, the large difference in the time scales of the wings and body motion results in the body sensing only the stroke averaged changes in the wing kinematics and the flight force [Bergou et al., 2010, Dickinson, 2005]. Thus, an aerial maneuver is executed by incrementally modulating the wing kinematics on a stroke-by-stroke basis. On the contrary, in maneuvering flight of damselflies, the body motion changes substantially within one wingbeat. This is evident from the short duration of the maneuvers in wingbeat time. Consequently, within-wingbeat changes in the wing kinematics are necessary to execute a maneuver.

Having independent control over the motion of all four wings, a damselfly can potentially use a variety of wing kinematics adjustments to execute a yaw turn. Visual inspection of Figure 5.9 illustrates this point immediately. Although the body kinematics of these turns is very similar, the details of the wing kinematics were different for each insect. These differences may have arisen from the differences in the wing and the body morphology. For instance, the hindwings of damselfly \#2 were lagged behind the forewings by $90 \mathrm{deg}$ meaning that the forewing and hindwings flap out of phase. The average phase difference was only about 25 and 15 deg for damselfly \#1 and \#3, respectively. Among other differences in the wing motion across the species is the extreme variation in the wing flapping angles that was measured in the yaw turn of damselfly $\# 3$. Both the largest and smallest flapping amplitudes were found in the flight data of this insect with the flapping amplitude of the left forewing in the first downstroke of the maneuver (129 deg) being the largest, and that of the right hindwing during the second downstroke $(34 \mathrm{deg})$ being the smallest flapping amplitudes that were measured in this experiment. Also, this damselfly (\#3) showed very little variations in the wing deviation angle after the first stroke. This was especially in contrast with damselfly \#2 which continued to alter the wings' deviation angle while the variations in the wing flapping amplitude were more moderate. The wing kinematics of damselfly \#1 was in between these two extreme by showing moderate variations in both flapping amplitude and deviation angle.

Nevertheless, our detailed investigation on the flight data showed that despite the aforementioned differences, there are common patterns in the coordination between the wings among the species. 



Figure 5.6: Wing kinematics of damselflies yaw turn. Kinematics of the fore and hind wings is shown in separate plots with solid and dashed lines, respectively. The inner and outer wings kinematics is indicated by red and blue. The instantaneous difference between Euler angles of the bilateral wings is shown in solid black. In this plot flap angle is positive when wing moves forward. The positive direction for deviation and pitch angles are moving up and pitching up, respectively. These plots show that different species of damselflies use their wings in distinct ways to perform a similar aerial maneuver. 



Figure 5.7: Wing kinematics of damselflies yaw turn. Kinematics of the fore and hind wings is shown in separate plots with solid and dashed lines, respectively. The inner and outer wings kinematics is indicated by red and blue. The instantaneous difference between Euler angles of the bilateral wings is shown in solid black. In this plot flap angle is positive when wing moves forward. The positive direction for deviation and pitch angles are moving up and pitching up, respectively. These plots show that different species of damselflies use their wings in distinct ways to perform a similar aerial maneuver. 



Figure 5.8: Wing kinematics of damselflies yaw turn. Kinematics of the fore and hind wings is shown in separate plots with solid and dashed lines, respectively. The inner and outer wings kinematics is indicated by red and blue. The instantaneous difference between Euler angles of the bilateral wings is shown in solid black. In this plot flap angle is positive when wing moves forward. The positive direction for deviation and pitch angles are moving up and pitching up, respectively. These plots show that different species of damselflies use their wings in distinct ways to perform a similar aerial maneuver. 




B



Figure 5.9: Wing tip trajectory in the body fixed coordinate system for damselfly \#2. (A) and (B) respectively show the perspective and back view of the wing tip trajectories. The fore and hind wings' trajectories are shown by black and purple lines. The initial position of the wing tip is shown by a sphere. It is evident that the wing trajectory is not periodic and varies significantly during the yaw turn. 
To better understand how damselflies steer a yaw turn, we plotted the half-stroke-averaged wing kinematics of the outer wings with respect to the inner wings (Figure 5.10). A total of 120 half strokes were analyzed; 10 half strokes per wing per insect. For the fore and hind pairs of the wings the connection of the inner and the outer wings are studied within the same pair. The data points from each individual's flight are shown with a different color and symbol shape in Figure 5.10. The linear regression analysis is applied to the complete data set including the data points from all three flights (120 half-strokes). The bilateral flapping amplitudes were found to be correlated. The amplitude of the outer wings was on average $10 \%$ smaller than that of the inner wings. This is in contrast with the patterns observed in aerial yaw turns of fruit flies which increase the flapping amplitude of the outer wing to generate yaw torque [Dickinson, 2005, Fry et al., 2003]. Similarly, few measurements on the wing kinematics of dragonflies in yaw turn suggest that the outer wing flaps with larger amplitude than the inner wing [Alexander, 1986]. The wing pitch angle is representative of the geometric angle of attack of the wing and was found to be higher for the inner wings of damselflies than the outer wings. In smaller insects such as flies, the generation of yaw torque was revealed to be tightly linked to the wing pitch angle [Bergou et al., 2010]. In addition, the inner wings' deviation angles were higher than those of the outer wings, with damselfly \#2 deviating from this trend the most.

Subsequently, the angular velocities of damselfly wings were investigated. A strong correlation was found between the velocity of the inner and outer wings (Fig. 6B) with the bilateral flapping velocity showing the strongest correlation $\left(R^{2}=0.97\right)$. The flapping velocities of the outer wings were about $9 \%$ smaller than those of the inner wings. This figure also shows that damselflies flap faster during downstroke when compared to the upstroke; the magnitude of the positive flapping velocities was larger than that of the negative velocities. The deviation and pitching velocities of the bilateral wings were strongly correlated as well. The outer wings' deviation velocities were less than $70 \%$ of the inner wings'. Our results imply that damselflies regulate the wing motion through its orientation as well as velocity during yaw turns. However, the existence of these correlations between the bilateral wing kinematics does not guarantee their role in steering the flight. This can only be revealed by analyzing the yaw torque generated by each wing and investigating the connection between the torque and the wing kinematics. This analysis is carried out in the next section. 
A
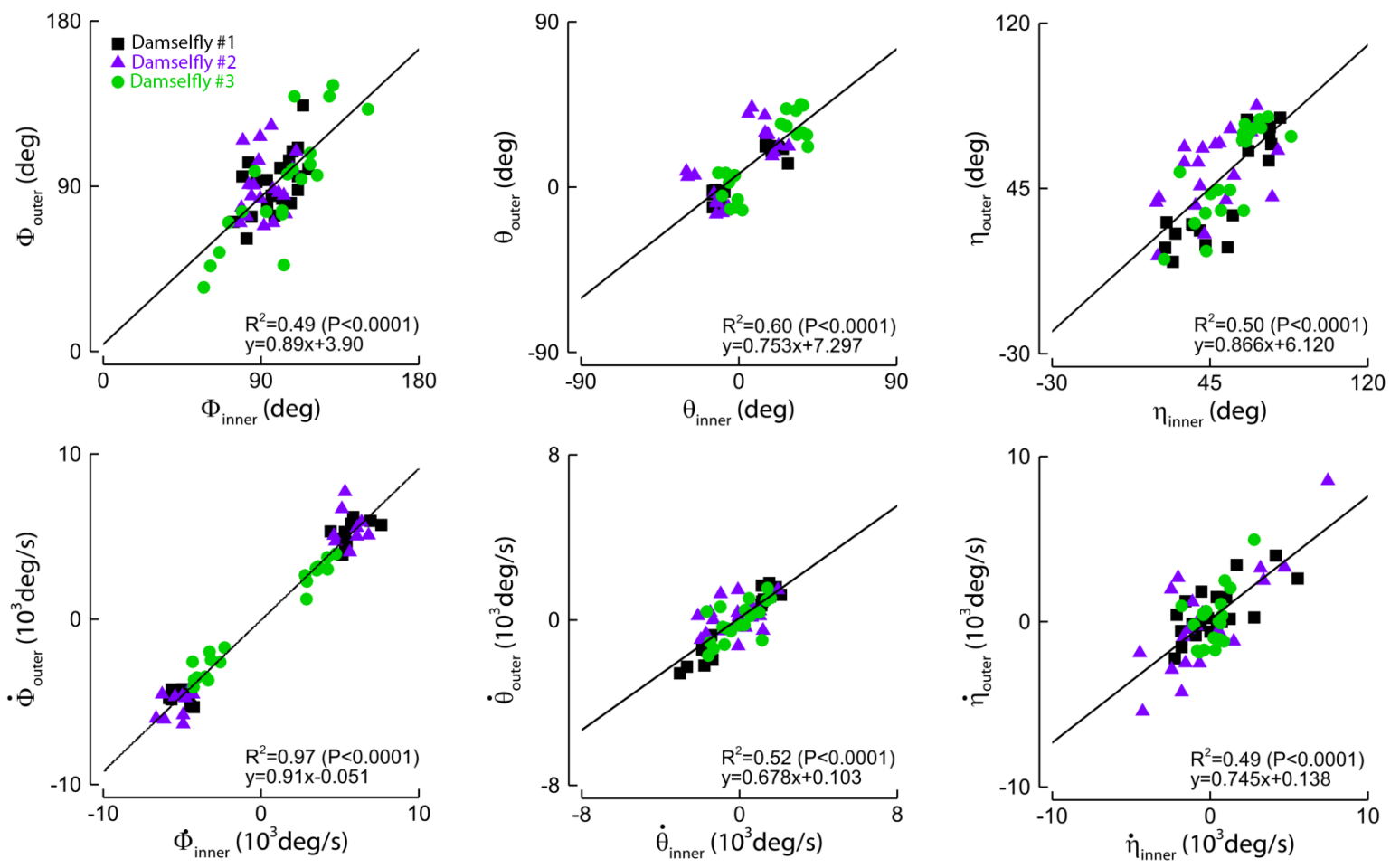

Figure 5.10: Correlation between the kinematics of bilateral wings. The half-stroke-averaged kinematics of the outer wings is plotted against the inner wings for all three flights. For clarity the data points form damselflies $\# 1, \# 2$ and $\# 3$ are indicated by black, purple and green, respectively. The linear regression line is shown in solid black. (A) The flapping amplitudes of the inner and outer wings were correlated, with inner wings having slightly higher amplitude than the outer wings. Similarly, the average magnitude of the deviation and the pitch angles of the inner wings were found to be higher than that of the outer wings. (B) The rates of change in all three Euler angles of the wings were bilaterally correlated, as well. The data also shows that the velocity of the inner wings is in general higher than that of the outer wings. 


\subsection{Aerodynamic yaw torque generation}

To investigate the aerodynamics of damselfly yaw turn, we used a quasi-steady force prediction model to calculate the aerodynamic force and torque generated by each wing. Note that the body yaw axis (z-axis) is pointing downward and therefore a negative torque causes a rotation to the left. Torque is non-dimensionalized by the product of body weight and body length. It was found that both pairs of the wings actively contribute to the yaw torque generation. However, there are some differences across the species. For instance, for damselflies $\# 1$ and $\# 3$, there was higher torque generation during upstroke when compared to downstroke. This was only the case for the forewings of damselfly $\# 2$. The magnitude of the non-dimensional torque was comparable for all three insects regardless of the obvious differences in their wings and body morphologies. As expected, a large yaw torque was generated during the first stage of the maneuver. Afterward, the amplitude of the generated torque dropped and the average magnitude of the torque shifted toward positive values. This is consistent with the observed reduction of body yaw velocity during the deceleration phase.


Figure 5.11: Aerodynamic yaw torque. The aerodynamic yaw torque generated by each wing is non-dimensionilized by The peak value of the torque is higher at the first stage of the maneuver. The sign of the yaw torque generated by the outer wings were reflected to achieve consistency in the data. Since the sign difference in the yaw torque generated by left and right wings is due to the respective location of the wing with respect to the center of mass, it is independent of the wing kinematics. Therefore, this adjustment does not affect the accuracy of our analysis. (A) and (B) respectively show the correlation of the magnitude of the yaw torque generated by outer versus inner wings and hindwings versus forewings.

To investigate the possible differences in the role of the fore and hind wings as well as the inner 
and the outer wings, we plotted the half-stroke-averaged yaw torque generated by the outer wings versus that generated by the inner wings in Figure. A strong correlation was found between these values with the outer wings generating about $70 \%$ of the torque produced by the inner wings. This implies that inner wings carry about $59 \%$ of the aerodynamic load caused by yaw torque generation. This is compatible with our observation of flight data which showed that the inner wings have higher average velocity then the outer wings throughout the maneuver (Figure 5.10). However, these results are in contrast with what was observed in yaw turns of smaller insects such as fruit flies. In fact, the stroke-averaged yaw torque generated by the inner wing of a fruit fly were found to be negligible compared to that of the outer wings [Bergou et al., 2010]. We also plotted the half-stroke-averaged yaw torque generated by the hindwings against that of the forewings (Figure 5.11). These values are found to be strongly correlated with the hindwings generating more than $120 \%$ of the torque generated by the forewings. Based on the two graphs in Fig. $7 \mathrm{~B}$ and $\mathrm{C}$, we can conclude that, on average, the inner hindwing and outer forewing generate the maximum and minimum yaw torque during the maneuver, respectively.

To determine which parameters of wing motion damselflies use to regulate yaw torque, we correlated the half-stroke averaged yaw torque generated by each wing to the wing orientation and velocities (Fig. 8A). In these plots the sign of the yaw torque generated by the outer wings were mirrored to achieve consistency in the data. Since the sign difference in the yaw torque generated by left and right wings is only due to the respective location of the wing to the center of mass (left or right), it is independent of the wing kinematics. Therefore, this adjustment does not affect the accuracy of our analysis. A correlation was found between the yaw torque and the wing pitch angle. However, yaw torque was independent of the variations in the wing amplitude or deviation angle. In Fig. 8B we plotted the half-stroke-averaged yaw torque versus the wing angular velocities. These correlations show that the changes in the wing flapping and deviation velocities contribute substantially to the modulation of yaw torque. The strongest correlation was found between the yaw torque and the wing angle of attack (Figure 5.12) which is defined in Equation 5.4. This result is not surprising considering that the generation of the flight force is tightly associated with this angle. Moreover, similar trends were previously observed in yaw turns of other insects such as fruit flies [Bergou et al., 2010]. The question that still needs to be answered is how a damselfly regulates its wing angle of attack during a maneuver. In general, an insect could induce the angle of attack 
by pitching the wing about its axis of rotation (Figure 5.12). This is equivalent to modulating the wing pitch angle. Alternatively, the angle of attack of the wing can be altered by reorienting the incoming velocity to the wing (Figure 5.12). This can be achieved by modifying the relative magnitude of the vertical and horizontal velocity components of the wing. Both approaches result in modifying the relative angle between the wing chord and the direction of the incoming velocity. To investigate which approach a damselfly takes, we correlated the half-stroke-averaged angle of attack to the wing kinematics parameters (Figure 5.11). A strong correlation was found between the angle of attack of the wings and the wing pitch angle as well as the ratio of the wing's vertical to horizontal velocities, . These results imply that a damselfly combines the two aforementioned approaches to regulate the angle of attack as well as the yaw torque.

\subsection{Discussion}

To change the flight heading, an insect or bird could employ different strategies. One of the common types of turn is a banked turn in which the flight force is tilted towards the center of rotation to bend the flight path [Ros et al., 2015]. A banked turn is similar to what airplanes do to implement a large change in the flight course. Alternatively, an insect could change the flight heading by executing a yaw turn. Unlike a banked turn, that consists of two separate tasks of redirecting the flight path and reorienting the body, in a yaw turn both tasks can be achieved by generating yaw torque in one direction. Our observation shows that in the absence of any external stimulus, damselflies are more likely to execute a yaw turn. The measurements on three different species of damselflies indicated that these insects employ a variety of changes in the wing kinematics to execute a yaw turn. The motion of each of the four wings changed significantly throughout the maneuver. These changes were found to be precisely coordinated through both the wing angles and angular velocities. Yet, only the alternations in the angular velocities were correlated with the yaw torque generation, as evident in Figure 5.12. These results do not dispel the effect of the wing orientation on the generation of aerodynamic yaw torque. Rather, they imply that the wing angular velocities are dominantly regulated by damselflies to alter the yaw torque. The wing angular velocity influences the torque generation by modifying both the magnitude and direction of the incoming velocity to the wing. The latter alters the wing angle of attack. The angle of attack of the inner wings were 
A
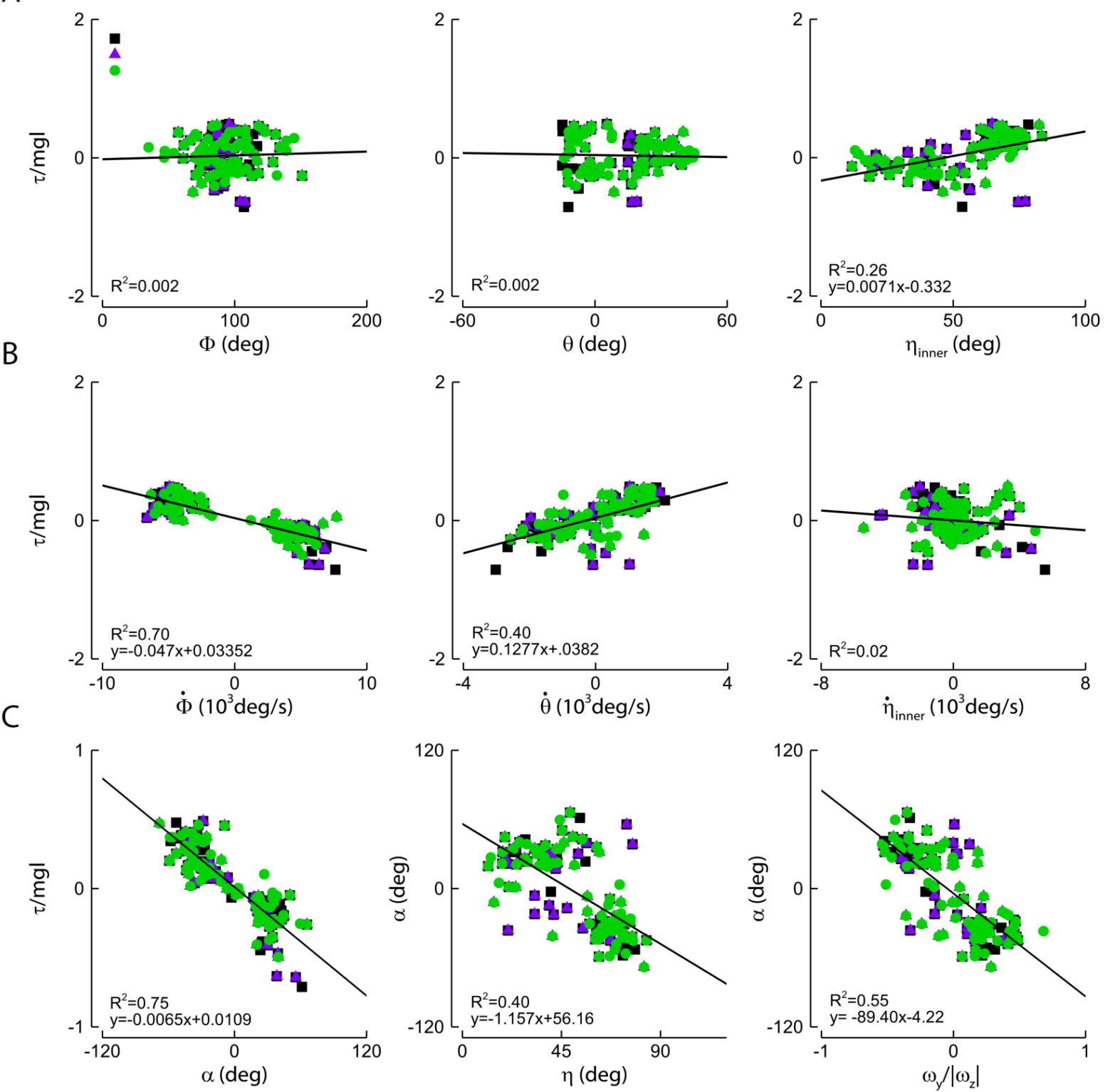

Figure 5.12: Mechanism of torque generation in damselfly yaw turns. For clarity the data points form damselflies $\# 1, \# 2$ and $\# 3$ are indicated by black, purple and green, respectively. The linear regression line is shown in solid black. (A) The half-stroke-averaged yaw torque is plotted against the half-stroke averaged angle of attack of the wings for all three flights together. A strong correlation was found between these two variables which suggest that the damselflies adjust the wing angle of attack to regulate the yaw torque. The half-stroke-averaged wing angle of attack of the wing is plotted against the half-stroke-averaged geometric angle of attack of the wing (B) and the ratio of wing's angular velocity about y and z axes, $\omega_{y} /\left|\omega_{z}\right|(\mathrm{C})$. The correlation between the angle of attack and $\omega_{y} /\left|\omega_{z}\right|$ was significantly stronger the correlation between this variable and the geometric angle of attack of the wing. 
found to be strongly correlated to the outer wings' $\left(R^{2}=0.80\right)$ with the angle of attack of the outer wings being less than $80 \%$ of that of the inner wings. Taken together, these results reveal important similarities and differences between damselflies and smaller insects such as fruit flies in the way that they execute a yaw turn. Although both insects rely on modulating the wing angle of attack and the incoming velocity, the mechanisms by which they implement these regulations are different. Similar to flies, damselflies alter the wing angle of attack by modulating the wing pitch angle. However, our results show that the variations in the wing velocity contribute substantially to the angle of attack regulation, as well. In addition, the yaw turns of damselflies are mostly driven by the inner wings, as is evident from the relative kinematics of the bilateral wings (Figure 5.65 .7 ??). This trend is in contrast to what was observed in the yaw turns of flies [Fry et al., 2003, Bergou et al., 2010]. Furthermore, unlike flies, damselflies apply drastic rather than subtle changes to the wing kinematics to execute an aerial yaw turn. We believe that this difference stems from the dissimilarities in the wing flapping frequency of these insects $(200-250 \mathrm{~Hz}$ for flies and 15-25 Hz for damselflies). For instance to change the flapping velocity as much as $500 \mathrm{deg} / \mathrm{s}$, a damselfly needs to alter the wing amplitude $25 \mathrm{deg}$. On the other hand, a fly will only need $2.5 \mathrm{deg}$ alternation in the wing flapping amplitude to achieve the same effect.

In continuation of our analysis, we calculated the yaw torque generated only by the wings, when the body motion was artificially extracted. This helps us understand the extent to which damping due to the body rotation affects the dynamic of the yaw torque [Hedrick et al., 2009]. Results show that the wings continue to generate yaw torque to turn the body to the left, throughout the maneuver. No evidence of counter torque generation was found in the torque produced by the wing motion only. However, when the body motion was added, the average torque was significantly shifted to positive values. Further analysis shows that the body motion affects the torque generation by two means; modifying the incoming velocity to the wings and altering the wing angle of attack. The first effect is known as flapping counter torque (FCT) which causes exponential reduction in the yaw velocity [Hedrick et al., 2009]. However, the second effect is unique to damselflies and potentially other low flapping frequency insects. On average the body motion reduced the wing angle of attack to $83 \%$ of its magnitude without the body motion $\left(R^{2}=0.89\right)$. This is because the rotational velocity of the body and the wings are close in magnitude. The ratio of the body yaw velocity to the wing flapping velocity reaches about 0.5 during the maneuver. For flies this ratio is only about 0.1-0.2 
based on the type of the motion [Muijres et al., 2014, Hedrick et al., 2009, Bergou et al., 2010]. Yet, the modulations in the wing angle of attack due to the body motion were not always negative. The motion of the body sometimes enhanced the asymmetry in the bilateral angle of attacks. Taken together, our results suggest that the dynamics and aerodynamics of aerial maneuvers of low flapping frequency insects may differ from those of high flapping frequency insects.

\subsection{Acknowledgement}

The 3D surface reconstruction of the damselfly wing and body was done by Ayodeji Bode-Oke. He also calculated the wing area as well as the body moment of inertia. 


\section{Chapter 6}

\section{Integrating wing into the dynamics of flapping flight}

\subsection{Introduction}

When the motion of the insect body changes, due an internal or external cause, the sensory system of the animal senses these changes. The sensory feedback is then interpreted by the brain (control system) and appropriate control signal is sent to the flight muscle altering their driving force or the configuration of the wing hinge. As a result the motion of the flapping wings changes leading in altering the aerodynamic force and consequently adjusting the body motion. The aforementioned cycle of actions represents the classic model of the closed loop dynamics of insect flight that was commonly used in previous studies [Cheng and Deng, 2011, Dickinson et al., 2000]. A schematic of the flight architecture based on this model is shown in Figure 6.1. All elements of flight are connected via a single loop in this model. The difference between this model and that of a fixed wing flier is that the control signal is implemented via changing the wing motion rather than the orientation of the control surfaces on the wing. Yet, in both models the only role of the wings is to generate the aerodynamic force and that is why it is identified by the "aerodynamics" block in the closed loop model of the flight (Figure 6.1).

Alternatively, we proposed a new model that recognizes the wing as a dynamic system that is forced by the flight muscles from within the thorax but also interacts with the surrounding air 




Figure 6.1: Conventional model of the flapping flight dynamics.

(Figure 6.2). In this model, the wing not only generates the aerodynamic force (the aerodynamic block in the conventional model) but also responds to the changes in the body motion or the external environment. The input to the aerodynamic block in the conventional model is no longer the same as the input to the wing block in the new model, meaning that the wing kinematics may be different from that which was enforced by the flight muscles. This results in an extra inner loop in the dynamics of the system which allow interactions between the wing and the body motion without passing through the outer loop of the system where the motion of the body can only affect that of the wing through the control signal generated by the brain. Existence of such an inner loop have several consequences on the dynamics of the flapping flight including its stability, maneuverability and wing kinematics control. As the time scales of the wing and the body motions gets closer, in low flapping frequencies or high body velocities, these coupling effects get potentially more severe resulting in a behavior that diverges from what the old model predicts.

In the next two chapters we will computationally and mathematically build the new model of the insect flight dynamics, focusing on the inner loop between the wing and the body dynamics. The model of the wing integrates the structural properties of the wing and its hinge with the unsteady aerodynamics of the flight. In the current chapter, we will focus on building this model, by developing appropriate tools for prediction of the aerodynamic torque about the wing hinge, modeling the rigid wing dynamics and incorporating the structural properties of the wing hinge. 


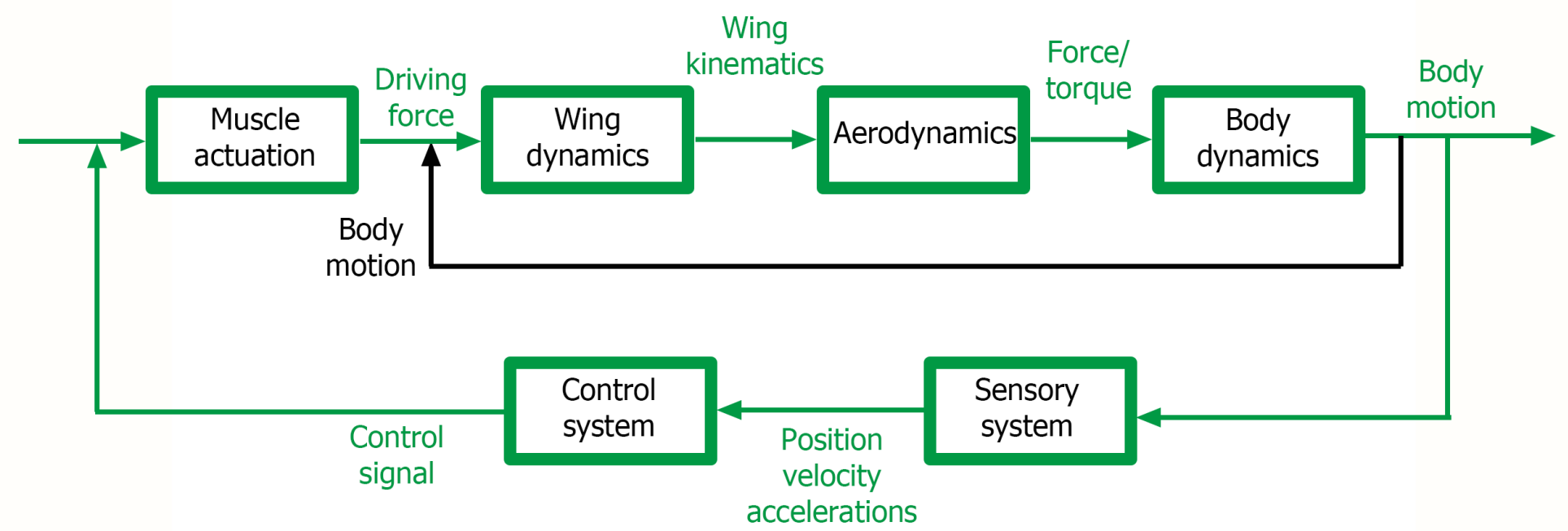

Figure 6.2: Proposed model of the flapping flight dynamics. Newly proposed model of the flapping flight in which the wing is separated from the aerodynamics block recognizing the wing as a dynamic system. The most important feature of this model is the extra inner loop that considers potential coupling between the dynamics of the wing and the body. This model allows the motion of the body to affect the motion of the wing without passing through the other elements of flight such as sensory feedback or the control system.

We show that the wing kinematics vary by the ratio of the aerodynamic to elastic forces in this model, providing a baseline for controlling the flight force in flight. This model is then used to study potential coupling between the wing and body kinematics. In the next chapter, we show that this model can accurately predict the dynamics of fast yaw turns in low flapping frequency insects such as damselflies, while the predictions of the old model significantly diverges from the observed behavior.

\subsection{Wing morphology and kinematics}

The wings of insects vary in shape and size. A generic wing shape is shown in Figure 6.3. The coordinate system of the wing is fixed on it and moves with it. The x-axis coincides with the wing's axis of rotation. To predict force and torque acting on the wing using a quasi-steady model we employed Blade Element method which assumes that the wing is consists of finite number of chordwise stripes that have no aerodynamic interactions (Figure 6.3). The local chord length at 


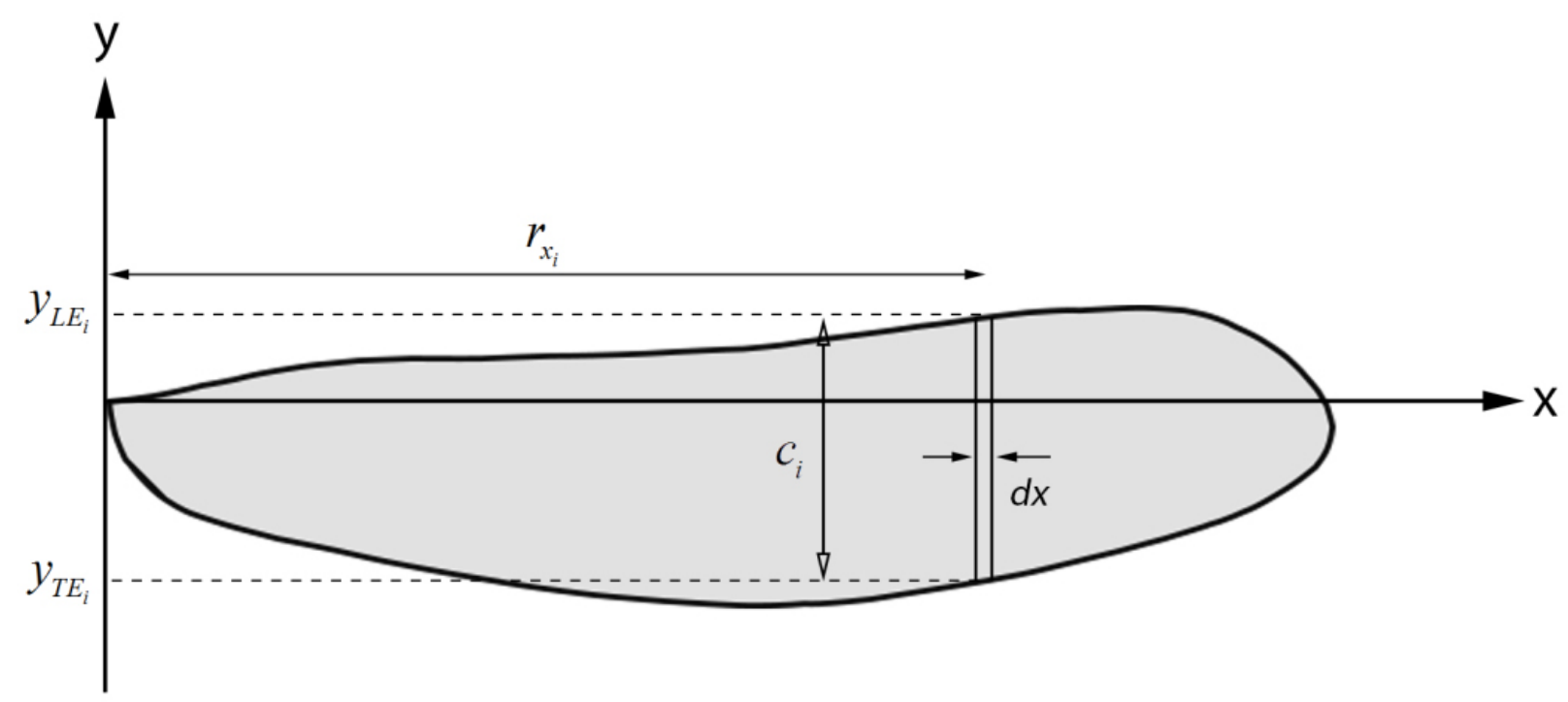

Figure 6.3: Insect wing model. A generic wing shape is shown. $y_{L E_{i}}$ and $y_{T E_{i}}$ define the distribution of the leading and trailing edge displacement from the wing axis of rotation. Blade element method decomposes the wing to many thin strips. $c_{i}$ is the wing chord at distance $r_{x_{i}}$ from the wing hinge.

each strip is calculated by Equation 6.1.

$$
c_{i}=y_{L E_{i}}-y_{T E_{i}}
$$

where $y_{L E_{i}}$ and $y_{T E_{i}}$ define the distribution of the displacement of the leading and trailing edges from the axis of rotation. An image of the real insect wing was digitized and the lengths along the wing span and chord were normalized by the wing length and the maximum wing chord,respectively. A $4^{t h}$ order polynomial curve is then fitted to the leading and trailing edges of the wing separately defining $y_{L E_{i}}$ and $y_{T E_{i}}$. To achieve realistic wing shape, wing length and area were matched to the real insect data. Axis of rotation always passes through the origin of the wing coordinate system. The amplitude of the leading and trailing edge curves of the wing (in y-direction in Figure 6.3) was scaled by a multiple of the mean chord ( $a \bar{c}$ for leading edge and $(1-a) \bar{c}$ for trailing edge) which represents relative location of the axis of rotation. By varying $a$ between 0 and 1 , the axis of rotation can be moved from leading edge to the trailing edge.

Kinematics of the wing is defined by three Euler angles representing the wing-fixed coordinate system $(x y z)$ relative to a reference coordinate system fixed on the stroke plane, $X Y Z$. Let $\psi, \theta$ 
and $\phi$ be the wing Euler angles that are conventionally called flapping, deviation and pitch angles, the angular velocity of the wing in the wing coordinate system can be calculated based on the Euler angles and their time derivatives as follows (Equation 6.2).

$$
\begin{aligned}
\omega_{x} & =\dot{\psi}-\dot{\phi} \sin \theta \\
\omega_{y} & =-\dot{\phi} \cos \theta \cos \psi+\dot{\theta} \sin \psi \\
\omega_{z} & =\dot{\phi} \cos \theta \sin \psi+\dot{\theta} \cos \psi
\end{aligned}
$$

In insect wings, there is evidence of significant aeroelastic effects in both local deformation of the wing surface [Daniel and Combes, 2002] and the gross rotation of the wing [Ennos, 1988, Bergou et al., 2007]. Here, we assumed that all the torsional elasticity of the wing is bulked at the wing hinge. Experiments have shown that having a rigid insect wing model with an elastic hinge (under dynamically similar condition) can reproduces the typical wing kinematics of an insect wing maintaining high angle of attack during flapping translation and rotate passively at stroke reversal. This wing was also able to generate comparable lift forces as that of the insect wing [Ishihara et al., 2009]. Elastic torque about the wing axis of rotation is proportional to the wing pitch angle.

$$
\tau_{\text {elastic }}^{\prime}=-G \Delta \psi
$$

$\Delta \psi=\psi-\psi_{0}$ and $\psi_{0}$ is the rest angle of the torsion spring. $G$ is the stiffness of the wing hinge structure.

\subsection{Dynamics of the wing pitching}

Among the three rotational degrees of freedom of a flapping wing, pitch angle is more significantly affected by the interactions of the wing with the air. In fact there is a growing body of evidence that supports passive maintenance of the wing angle of attack [Ishihara et al., 2009] as well as passive wing rotation at stroke reversal [Bergou et al., 2007, Ennos, 1988] . It was initially suggested that the inertial forces governs wing pitching (rotation) at stroke reversal. It was recently shown that the added mass from the surrounding fluid can rotate a wing, which is elastically hinged to the body, even in the case of reduced inertial effect of the wing [Ishihara et al., 2009]. In another experiment on the passive rotation of a drone fly wing model, it was suggested that the inertial effects plus the 
torque resulting from deviation of the center of pressure of the translation force from the axis of rotation can capture the main features of the wing rotation angle [Whitney and Wood, 2010].

Insects though are capable of actively controlling wing pitching [Dudley, 2002]. Asymmetry in bilateral wing pitch angle, in fact, plays a significant role in generating flight torque and therefore performance in the aerial maneuvers [Bergou et al., 2010, Dickinson et al., 1993, Fry et al., 2003]. Yet, it is not well understood whether the active torque exerted by the flight muscles to rotate the wing is large enough to completely flip the wing or if their role is more to manipulate the wing trajectory for control purposes. It is known, however, that both capabilities exist, at least, in some insects and therefore it is important to investigate how it can affect control of the flight. It was previously suggested [Bergou et al., 2010] that insects might combine active and passive wing kinematic changes in order to perform a turn maneuver. In this theory, asymmetry in wing pitch angle was indirectly induced by actively changing properties of the elastic material in the wing hinge.

To gain insight to the potential role that passive and active wing pitch can play in the maneuverability of the insects, we constructed an insect wing model with passive pitching capability. Aerodynamics pitch torque about the wing axis of rotation is predicted by a quasi-steady model, inspired by unsteady airfoil theory. Prediction of the passive wing pitch angle as well as the aerodynamic pitching torque was shown to be in excellent agreement with experimental measurements. Keeping symmetric wing kinematics, we then studied how wing pitch angle responds to the body motion in maneuver. Our methodology isolates potential effect of the wing hinge torsional flexibility from that of the active manipulation of this angle by control muscles. Our results show that in a fast yaw turn maneuver pitch angle of the inner and the outer wings vary asymmetrically. We generalized our results by a systematic investigation on the effect of two non-dimensional numbers; wing aspect ratio, AR, and the ratio of the body angular velocity to the average wing angular velocity,$\Omega / \omega$.

Insect wings flex and deform under the action of aerodynamic and inertial forces [Combes and Daniel, 2003] [Koehler et al., 2012]. In this study however, we assumed that the wing is rigid and hinges to the body at the wing shoulder. Therefore equations of motion of the rigid body can be applied to the wing motion, which is entirely rotational. We also assumed that flapping and deviation angles are prescribed which leaves us with only one degree of freedom of the wing, namely, wing pitching. In 
the wing coordinate system, which co-rotates with the wing, Equation 6.4 describes wing pitch, $\psi$, about the wing pitching axis.

$$
\begin{gathered}
I_{x x} \dot{\omega}_{x}+\left(I_{z z}-I_{y y}\right) \omega_{y} \omega_{z}+I_{x y}\left(\dot{\omega}_{y}-\omega_{x} \omega_{z}\right)+ \\
I_{y z}\left(\omega_{y}{ }^{2}-\omega_{z}{ }^{2}\right)+I_{x z}\left(\dot{\omega}_{z}+\omega_{x} \omega_{y}\right)=\tau_{\text {aero }}+\tau_{\text {elastic }}
\end{gathered}
$$

$\left(\omega_{x}, \omega_{y}, \omega_{z}\right)$ is the vector of the angular velocity of the wing in the wing coordinate system. $\tau_{a e r o}$ and $\tau_{\text {elastic }}$ respectively are the aerodynamic and elastic reaction torques about the axis of rotation of the wing. $I_{i j}$ is the tensor of moment of inertia of the wing in the wing coordinate system. It was calculated assuming homogeneous distribution of mass across the wing area. Both the wing and the body coordinate systems are right-handed and are shown in Fig 1. The coordinate system of the body is attached to the stroke plane in such way that $x_{s} y_{s}$ lays in this plane. $z_{s}$ is normal to the stroke plane and points upward. The origin of this coordinate system is at the wing base. Kinematics of the wing is specified by flapping, deviation and pitch angle of the wing which are Euler angles about $z_{s}, y_{s}$ and $x_{s}$ respectively.xyz coordinate (wing coordinate system) rotate with the wing. $y$ points to the leading edge and $x$ points upward. $x$ is the wing pitching axis. Elastic torque about the wing axis of rotation is proportional to the wing deflection.

$$
\tau_{\text {elastic }}=-G \Delta \psi
$$

$\Delta \psi=\psi-\psi_{0}$ and $\psi_{0}$ is the rest angle of the torsion spring. $G$ is the stiffness of the wing hinge structure.

\subsection{Aerodynamic pitching torque model}

In general, there are two categories of aerodynamic torque acting about the wing's axis of rotation. The first category is the torque due to the displacement of the center of action of the aerodynamic force from the rotation axis. The other category is pure torque terms which are usually caused by mutually opposing forces. Therefore, the effect of these forces on the overall movement of the object is difficult to observe. Commonly, in previous studies only the first category of the torque terms were used to predict the aerodynamic torque exerted on a flapping wing (by the added inertial effect 
being an exception to that)[Whitney and Wood, 2010, Bergou et al., 2010]. Alternatively, the newly proposed model includes a pure circulatory rotational torque term which was found to be essential for accurate prediction of aerodynamic pitching torque. This term is exceptionally important when the rotation axis is located far from the wing's geometric center and close to the leading edge, as it is the case for insect wings.

The pitching torque model proposed here is based on the Theodorsen's solution for a 2 dimensional pitching-plunging foil. Appropriate modifications made then made to incorporate aerodynamics of flapping flight. Applying blade element method, the flapping wing is divided to a finite number of chordwise strips and the pitching torque is estimated based on the local flow condition (Fig). Any spanwise interactions between the stripes are assumed negligible. The total torque on a $2 \mathrm{D}$ strip is sum of four terms, added mass (am), translational (trns), rotational (rot) and dissipative (diss).

$$
d \tau_{\text {aero }}=d \tau_{\text {am }}+d \tau_{\text {trns }}+d \tau_{\text {rot }}+d \tau_{\text {diss }}
$$

The total torque on the wing can be calculated by integration over the wing span, $\tau_{\text {aero }}=$ $\int_{0}^{R} d \tau_{\text {aero }} d r$. Equation 6.7 represents the expression of each one of the terms.

$$
\begin{aligned}
& d \tau_{a m}=-I_{a} \dot{\omega}_{x}+\frac{1}{4} \pi \rho_{f} c^{2}\left(c_{i} / 2-x_{i}\right) \dot{v}_{y} d r \\
& d \tau_{\text {trns }}=\rho_{f} \Gamma_{t r n s} v_{x}\left(x_{i}-x_{C p_{i}}\right) d r \\
& d \tau_{r o t}=-\rho_{f} \Gamma_{r o t} v_{x}\left(x_{C p_{i}}-x_{i}\right)-\frac{1}{2} \rho_{f} \Gamma_{r o t} v_{x}\left(0.75 c_{i}-x_{i}\right) d r \\
& d \tau_{d i s s}=d F_{y}^{\nu}\left(x_{i}-x_{C p_{i}}\right)-\frac{1}{8} \rho_{f} C_{r d}\left|\omega_{x}\right| \omega_{x}\left(y_{L E_{i}}\left|y_{L E_{i}}{ }^{3}\right|-y_{T E_{i}}\left|y_{T E_{i}}{ }^{3}\right|\right) d r
\end{aligned}
$$

$C_{r d}$ is the rotational damping coefficient. $c_{i}$ is the chord length of the $i^{\text {th }}$ cross section of the wing along the span and $x_{i}$ is the distance of the rotation axis from the section's leading edge. $y_{L E_{i}}$ and $y_{T E_{i}}$ are the amplitudes of the leading and the trailing edges of the $i^{\text {th }}$ cross section measured from the pitching axis (Figure 6.3). $v_{y}$ and $v_{x}$ are the wing velocity components along the $\mathrm{y}$ and $\mathrm{z}$ axes of the wing.

$x_{c p_{i}}$ represents the location of the center of pressure and was estimated by Equation 6.8 which was experimentally measured for a drosophila wing [Dickson et al., 2006]. In order to generate appropriate moments about the wing's rotation axis, the total force acting on each blade element is located at the center of pressure of that element which is a function of the local angle of attack, $\alpha$. 


$$
x_{c p_{i}}=\bar{c}\left(\frac{0.82}{\pi}|\alpha|+0.05\right)
$$

A short description on each component of torque is provided below.

\subsubsection{Added mass}

Added mass refers to the virtual Assuming a 2D wing section has negligible thickness, the added mass coefficients can be expressed as follows:

$$
I_{a}=\frac{1}{4} \pi \rho_{f} c_{i}^{2}\left[\frac{1}{32} c_{i}^{2}+\left(c_{i} / 2-x_{i}\right)^{2}\right]
$$

The torque due to the added mass has two components: rotational, which is proportional to the angular acceleration, and translation, which is resulted from the deviation of the rotation axis from the buoyancy center. The added mass is an unsteady aerodynamic effect which can potentially have significant contributions to the force and torque generation in flapping flight [Ellington, 1984b]. It was shown that added mass can cause leading edge flip at stroke reversal [Bergou et al., 2007, Ishihara et al., 2009].

The translational torque is the torque due to translational lift. Translational circulation has the same form as in [Andersen et al., 2005] and it repeated in Equation 6.10.

$$
\Gamma_{t r n s}=-C_{T} c_{i} v_{y} v_{z} / \sqrt{v_{y}^{2}+v_{z}^{2}}
$$

The strength of this torque term is proportional to the angle of attack since both the magnitude of the circulation as well as the moment arm of the translational force increase proportional to the angle of attack increment. Since the axis of rotation is forward to the center of pressure, this torque will pitch the wing down and therefore has stabilizing effect.

The rotational torque in Equation 6.7 term consists of two terms. The first term is the torque due to the action of the rotational lift. The rotational circulation is calculated using Equation 6.11.

$$
\Gamma_{r o t}=c_{r}\left(.75-\hat{x}_{0}\right) \dot{\psi} c^{2}
$$


$\hat{x}_{0}$ is the dimensionless distance of the rotation axis measured from leading edge in chord lengths. $c_{r}$ is a constant which was set to $\pi$. It was previously proved that this form for the rotational circulation shows good accuracy in describing the aerodynamics of flapping flight [Pesavento and Wang, 2004, Sane and Dickinson, 2002]. The second term in rotational component of the torque, is a pure torque term that is caused by the action of two mutually opposing forces and therefore has no counterparts in the force description [Bisplinghoff et al., 2013]. This term increases the overall contribution of the rotational circulation to the aerodynamic pitching torque without affecting the force. This is consistent with the previous studies that have shown that a higher rotational force is required to explain the measured aerodynamic torque [Bergou et al., 2007]. The combined action of the two rotational terms can be represented as if the effective center of action of the rotational lift was further behind the center of action of translational lift. That enhances the contribution of the rotational lift to the aerodynamic torque.

The last component of the torque is the dissipative torque which originates from the resistance of the air to the wing's translation as well as the rotation. The first term in dissipative torque description is the torque due to action of the drag force. The drag force is proportional to the square of the velocity and has the form as represented in Equation 6.12 [Morison et al., 1950, Andersen et al., 2005].

$$
F^{\nu}=\frac{1}{2} \rho_{f} c_{i}\left[C_{D_{0}} \cos ^{2}(\alpha)+C_{D_{\pi / 2}} \sin ^{2}(\alpha)\right] \sqrt{v_{y}^{2}+v_{z}^{2}}\left(v_{y}, v_{z}\right)
$$

The second term is the rotational damping term which arises from the pure rotational motion of the wing and its magnitude is proportional to the square of pitching velocity of the wing. At the stroke reversal, when the rotational velocity of the wing is high, the rotational damping torque plays an essential role in governing the dynamics of the wing pitching [Whitney and Wood, 2010, Pesavento and Wang, 2004].

\subsection{Validation of the prediction results with experiment}

Although there is a lack of direct measurement of the aerodynamic pitching torque about a flapping wing's rotation axis, there are several experimental and computational studies that report passive wing pitch angle. Here, we contrasted the prediction of our model against two experiments 
on passively pitching insect wing models [Ishihara et al., 2009, Whitney and Wood, 2010]. In both experiments, the wing is elastically hinged to an apparatus that enforces its flapping motion. Maintenance of the wing angle of attack during the translation phase as well as the leading edge flip at the stroke reversal is governed by the balance between the inertial, elastic and the fluid torque. Whitney and Wood [Whitney and Wood, 2010] measured the time march of the wing pitch angle of an in-scale drone fly wing model flapping at $100 \mathrm{Htz}$. They also studied the effect of the flapping angle profile as well as the hinge stiffness and flapping frequency. The computed wing pitch angle using the current model is contrasted against these 3 experiments in Figure 6.4. The excellent agreement that was found between the experimental and computational data implies that the robustness of the model to the changes in wing kinematics as well as the hinge stiffness.

\subsection{Numerical simulation of the wing-air interactions}

The governing equation of the dynamics of the wing needs to be solved numerically at each time step and the solution must be marched in time to get the time history of the wing pitch angle. To do so, we solved Equation 6.4 in the wing coordinate system. Assuming that the wing is thin (thickness $<<$ chord), $I_{y z}=I_{x z}=0$. Discretizing the total duration of the simulation into $\mathrm{N}$ time steps, we can solve for $\dot{\omega}_{x_{n}}$ using Equation 6.14. The algorithm bellow was followed to march from time $t_{n-1}$ to $t_{n}$ :

1- Set the initial value for pitch angle, velocity and acceleration to its calculated value from the previous time step: $\psi_{0}=\psi_{n-1}, \dot{\psi}_{0}=\dot{\psi}_{n-1}$ and $\ddot{\psi}_{0}=\ddot{\psi}_{n-1}$

2- Calculate $\omega_{n}$ using Equation

$$
\begin{aligned}
\omega_{x_{n}} & =\dot{\psi}_{0}-\dot{\phi} \sin \theta \\
\omega_{y_{n}} & =-\dot{\phi} \cos \theta \cos \psi_{0}+\dot{\theta} \sin \psi_{0} \\
\omega_{z_{n}} & =\dot{\phi} \cos \theta \sin \psi_{0}+\dot{\theta} \cos \psi_{0}
\end{aligned}
$$

Note that the values for flap and deviation angles and velocities are known since the flapping motion of the wing is prescribed.

3- Calculate the aerodynamic and elastic torque; $\tau_{\text {aero }}\left(\omega_{n}\right)$ and $\tau_{\text {elastic }}\left(\psi_{0}\right)$ 
Figure 6.4: Validation of the wing pitch angle prediction. The predicted (solid black line) and measured (closed circles) values of the passive wing pitch angle (black line) for a model hover fly wing are shown. The prescribed flap angle is shown with solid navy line. 
4- Solve the governing equation of the wing dynamics (Equation 6.14 ) to get $\ddot{\psi}_{n}$.

$$
\dot{\omega}_{x_{n}}=\frac{1}{I_{x x}-I_{a}}\left[\tau_{\text {aero }}+\tau_{\text {elastic }}+I_{x x} \omega_{y_{n}} \omega_{z_{n}}+I_{x y}\left(\dot{\omega}_{y_{n}}-\omega_{x_{n}} \omega_{z_{n}}\right)\right]
$$

where $I_{a}$ is the added mass coefficient calculated by Equation

$$
I_{a}=\int_{0}^{R} \frac{\pi \rho_{f}}{4} c^{2}\left[\left(c / 2-y_{L E}\right)^{2}+\frac{1}{8}(c / 2)^{2}\right]
$$

5- Calculate $\ddot{\psi}_{n}$

$$
\ddot{\psi}_{n}=\omega_{x_{n}}+\dot{\phi} \sin \theta
$$

6- Update the wing pitch velocity

$$
\dot{\psi}_{n}=\dot{\psi}_{n-1}+\left(\gamma \ddot{\psi}_{n}+(1-\gamma) \ddot{\psi}_{0}\right)
$$

where $\gamma$ is the relaxation factor that is used to control stability of the numerical scheme. For most of the simulations it was set to 0.5 .

7- Update the wing pitch angle

$$
\left.\psi_{n}=\psi_{n-1}+\ddot{\psi}_{n} d t+0.5 \ddot{\psi}_{n-1} d t^{2}\right)
$$

8- Calculate error; $\delta=\psi_{n}-\psi_{0}$

9- If $\delta>\Delta$ then set $\psi_{0}=\psi_{n}, \dot{\psi}_{0}=\dot{\psi}_{n}$ and $\ddot{\psi}_{0}=\ddot{\psi}_{n}$. Go back to 1 .

10- If $\delta<\Delta$ then $n=n+1$. Go back to 1 .

\subsection{Non-dimensional numbers governing the dynamics of the wing pitch}

The dynamics of the passive wing pitching is governed by combined effect of the wing morphology and structure, wing and body kinematics and the flow properties. A list of these parameters are 
provided below;

$c$ : Wing chord

$R$ : Wing length

$G$ : Hinge stiffness

$\rho_{w}$ : Wing density

$\rho_{f}$ : Fluid density

$\nu$ : Fluid viscosity

$f$ : Flapping frequency

$\Omega$ : Body rotational velocity

$\Phi$ : Flapping amplitude

$\Psi$ : Wing pitch angle

It is not immediately obvious if all these parameters have independent effect on the output. Dimensional analysis was performed to combine the effect of individual parameters in physically relevant non-dimensional quantities as listed below.

$$
\begin{aligned}
& \Pi_{1}=\frac{c}{R} \\
& \Pi_{2}=\frac{\Omega}{\omega} \\
& \Pi_{3}=\frac{\rho_{f} c^{5} f \omega}{G} \\
& \Pi_{4}=\frac{\rho_{f} c^{3}}{m_{w}} \\
& \Pi_{5}=\frac{U c}{v} \\
& \Pi_{6}=\frac{f c}{U}
\end{aligned}
$$

where $\Pi_{i}$ are the non-dimensional numbers, $\omega=2 f \Phi$ is the average wing flapping velocity and $U=\Phi f R$ is the wing tip reference velocity. $\Pi_{1}$ through $\Pi_{6}$ are respectively called Aspect ratio $(A R)$, Body to wing velocity ratio or Normalized body velocity $(\bar{\Omega})$, Cauchy number $(C h)$, Mass ratio $(M)$, Reynolds number $(R e)$ and Reduced frequency $(K)$.

Investigating the effect of all these non-dimensional quantities and their combinations is impractical. Effect of Re was eliminated from the analysis assuming that the flow phenomena does not change severely across the relevant range for insect flight. Besides, since we use a quasi-steady 
approximation of the aerodynamic force the viscous effects are absent in our analysis. To further simplify the analysis we assumed that the flapping amplitude is $100 \mathrm{deg}$. Nevertheless, the flapping amplitude of the insects varies among species; as low as 60 deg (author measurements) for dragonflies to $140 \mathrm{deg}$ for high flapping frequency insects such as fruit flies [Dickinson and Gotz, 1993]. Keeping flapping amplitude constant, $K$ and $A R$ are equivalent. In addition, Mass ratio reduces to $A R$ assuming that the wing thickness (set to $6.5 \mu \mathrm{m}$ ) and the wing density (set to $1200 \mathrm{~kg} / \mathrm{m}^{3}$ ) does not vary across species. Therefore, we are left with three independent non-dimensional numbers; $C h, A R$ and $\bar{\Omega}$.

In the next section we will investigate the effect of the $C h$ as well as $A R$ on the dynamics of the wing pitch in hover $(\bar{\Omega}=0)$. This allows us to characterize the dynamics of the passive wing pitch across a wide range of the insect wing morphology and kinematics. Next, we study the effect of the body motion on the wing dynamics.

\subsection{Passive wing pitching}

Insect wings come in different shapes and sizes. Insect wings length vary from a millimeter across to few centimeters. Similarly, the flapping frequency varies across several orders of magnitude among insects ranging between 1-1000 Hz. Fortunately, the effect of these parameters can be combined to only a few non-dimensional quantities that independently affect the wing pitch dynamics. The effect of the wing shape is studied via variations in the $A R$. Wing aspect ratio is a geometrical parameter and can be easily measured. It varies between 2-6 across different insect species. However, it is more difficult to estimate the range of variations in the $C h$ number for insects. This is mainly because there are only a few measurements of the insect wing hinge stiffness available. Table 6.1 shows magnitude of $C h$ for a several species of insects.

Based on our analysis, the dynamics of the wing pitch can be understood across different insect wing sizes, shapes, kinematics and structural properties by investigating the behavior of the system across a limited range of $C h$ and $A R$. For three different wing aspect ratios, 3, 4 and 5, we varied $C h$ between 1E-4 to $1 \mathrm{E}-1$ and plotted the average wing pitch angle (equivalent to the wing angle of attack since the deviation of the wing from the stroke plane was set to zero) as well as the normal and horizontal force coefficients which are defined by 


\begin{tabular}{cccccc}
\hline Insect & $c(\mathrm{~cm})$ & $f(\mathrm{~Hz})$ & $\Phi(\mathrm{deg})$ & $G(\mathrm{Nm} / \mathrm{rad})$ & $\mathrm{Ch}$ \\
\hline Fruit fly & $0.102^{1}$ & $200^{1}$ & $135^{1}$ & $5.73 \mathrm{E}-9^{1}$ & $4 \mathrm{E}-2$ \\
Crane fly & 0.262 & 45.5 & 123 & $2.2 \mathrm{E}-7^{2}$ & $6 \mathrm{E}-3$ \\
Blow fly & $0.33^{3}$ & $150^{4}$ & 130 & $1.27 \mathrm{E}-6^{5}$ & $3.7 \mathrm{E}-2$ \\
Hover fly & $0.34^{6}$ & $100^{6}$ & $130^{6}$ & $1.64 \mathrm{E}-6^{5}$ & $1.8 \mathrm{E}-2$ \\
\hline \hline
\end{tabular}

${ }^{1}$ Values are taken from [Bergou et al., 2010];

${ }^{2}$ Values are taken from [Ishihara et al., 2009];

${ }^{3}$ Values are taken from [Deng et al., 2006];

${ }^{4}$ Was estimated assuming that the wing aspect ratio is 3 . The wing chord is given in [Deng et al., 2006] to be $1 \mathrm{~cm}$.

${ }^{5}$ Values are taken from [Ennos, 1988];

${ }^{6}$ Values are taken from [Whitney and Wood, 2010];

Table 6.1: Cauchy number of several species of insects.

$$
\begin{aligned}
C_{N} & =\frac{F_{N}}{0.5 \rho_{f} S_{w} V_{r e f}^{2}} \\
C_{H} & =\frac{F_{H}}{0.5 \rho_{f} S_{w} V_{r e f}^{2}}
\end{aligned}
$$

where $V_{\text {ref }}$ is the velocity of the wing at the mid-span. $F_{N}$ and $F_{H}$ are the normal and horizontal components of the aerodynamic force.

Figure 6.5A shows the half-stroke-averaged wing pitch angle in the translational phase of the wing stroke as a function of $C h$ for three different wing $A R$. For fixed flapping frequency and wing morphology, increase in $C h$ can be interpreted as decrease in the structural stiffness of the wing hinge and increase in flexibility. Similarly, for fixed wing morphology and structural properties increase in $C h$ occurs with increase in the wing velocity. Two dimensional trajectory of a mid-span chordline of the wing is shown in Figure $6.5 \mathrm{~B}$ and $\mathrm{C}$, for two cases of large and small $C h$. As the strength of the elastic force relative to the aerodynamic force increases, wing translates with larger pitch angles through the stroke plane.

Next, we plotted variations of the half-stroke-averaged normal and horizontal force coefficient with $C h$ for different wing aspect ratios (Figure 6.6). $C_{N}$ increases with $C h$ till it reaches a maximum value. $C h$ corresponding to the maximum $C_{N}$ is a function of the wing shape, with lower aspect ratio wings achieving larger maximum $C_{N}$ at a higher $C h . C_{H}$ decreases by increase in $C h$ for all wing aspect ratios. The $C h$ measured in different insect species is shaded in Figure 6.6. This region is the high lift producing region with sharp changes in the force coefficient with $C h$. 
A

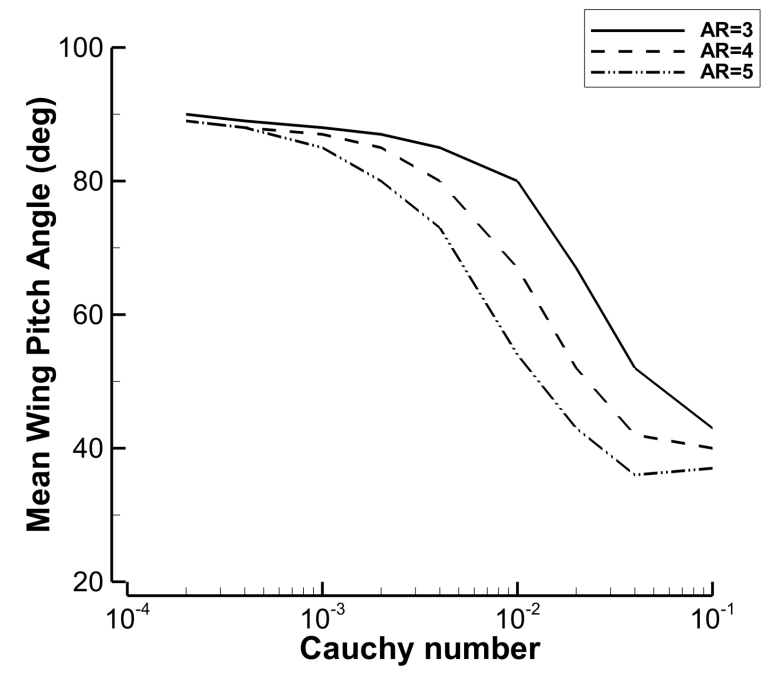

B

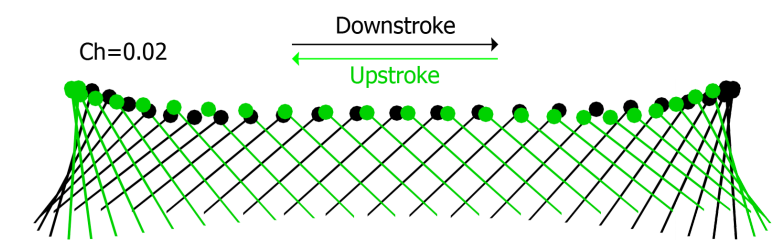

C

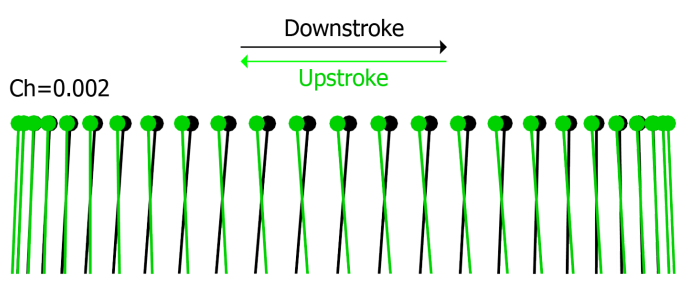

Figure 6.5: Dynamics of passive wing pitch across different insect wing kinematics and morphology. (A) Half-stoke-averaged wing pitch as a function of $C h$ is shown for three different wing aspect ratios. The trajectory of the midspan chordline is shown for two cases of large (B) and small (C) $C h$. Downstroke and upstroke are shown with two different colors. Stiffer wings have larger pitch angle during the translational phase of the wing stroke.
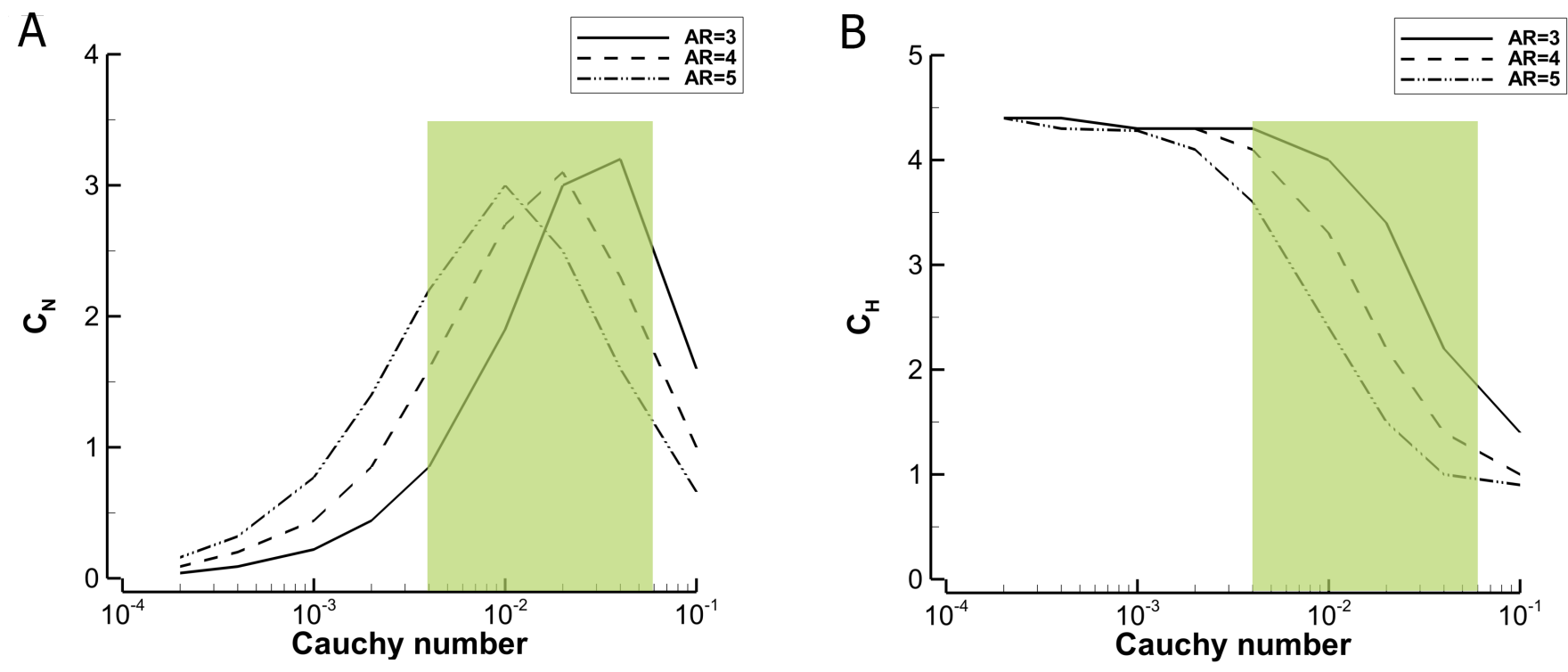

Figure 6.6: Variation of force coefficient with $C h$ for passively pitching wing. Variations of $C_{N}$ (A) and $C_{H}$ (B) with $C h$ for three different wing aspect ratios. The shaded area shows the range of measured $C h$ in insects. 
Our results show that across different wing morphology and kinematics, there is wide range of $C h$ numbers that allow maintenance of the high angle of attack as well as generation of high lift and thrust. In addition, these results suggest that by changing $C h$ an insect can control the wing kinematics as well as the flight force. $C h$ can be altered either by changing the wing hinge stiffness or the wing velocity (assuming that the wing geometry is fixed for an individual).

\subsection{Effect of the body rotations on the wing pitch}

The results of our analysis in the previous section show that the dynamics of the wing pitch is strongly affected by the relative strength of the aerodynamic and elastic force exerted on the wing. We showed that changes the wing kinematics or its structural properties alter the wing pitch angle as well as the flight forces. Besides changes in the wing kinematics, body motion can potentially affect the aerodynamic force generation. In high frequency flapping flight, the velocity of the wing is significantly larger than that of the body and therefore the effect of the body motion on the aerodynamic force generation is minor compared to that of the wing motion itself. For instance, a fruit fly flap its wings more than 200 times a second across a wide stroke amplitude of about 140 $\operatorname{deg}$ [Bergou et al., 2010]. That results in wing rotational velocities as high as 56,000 deg/s. The rotational velocity of the body even in fastest recorded aerial maneuvers barely exceed 5000-6000 $\mathrm{deg} / \mathrm{s}$ [Muijres et al., 2014] which is less than $10 \%$ of he wing velocity. Nevertheless, this is not the case for low flapping frequency insects. Despite the large changes in the flapping frequency across flying insect species, the variations in the body velocities are very limited. In fact, a study on the yaw turns of several flying animals show that in contrast to the considerable diversity in the wing and body morphology, they all exhibit a similar yaw peak velocities [Hedrick et al., 2009]. Therefore, as the flapping frequency decreases, the magnitude of the wing and body approach and therefore the aerodynamic effect of the body motion grows. For instance, the body velocity of a damselfly rises to about $1500 \mathrm{deg} / \mathrm{s}$ during yaw turns (refer to chapter 4) which is about 20 to $30 \%$ of the wing velocity. At such velocities, the rotation of the body changes the balance of the aerodynamic and elastic forces $(C h)$ and can potentially affect the dynamics of the wing pitch.

In this section, we examine the effect of lateral rotations of the body (meaning yaw and roll rotations) on the bilateral wing pitch angles. When an insect experiences a whole body rotation, in 
downstroke (upstroke), the net incoming velocity increases on the outer (inner) wing and it decreases on the inner (outer) wing. This results in asymmetric variation in the fluid dynamic pressure on the wings. Assuming that the elastic properties of the wing hinge remain unchanged, the relative strength of the fluid force (and torque) compared to the elastic reaction force, $C h$, varies. The asymmetric change in $C h$ number then causes asymmetric response of the wings pitch angles. For instance, on the outer wing during downstroke, the fluid forces are strengthened (since the center of pressure of the wing is located behind the axis of rotation)which results in stronger pitch down torque being exerted on the wing. Similarly, on the inner wing, the fluid forces are weakened and elastic forces are able to rotate the wing further upward which causes a higher pitch angle on this wing. However, the effectiveness of this phenomenon depends on the extent to which $C h$ number changes due to the body angular velocity. The relative change in $C h$ number of the bilateral wings depends on the relative magnitude of the angular velocity of the body compared to the wing.

$$
C h_{\text {maneuver }}=\frac{\rho_{f} L_{w} \bar{c}^{4} f}{G} \omega\left(1 \pm \frac{\Omega}{\omega}\right)
$$

We first examine the effect of the body yaw velocity. The difference in the pitch angle of the inner and the outer wings in downstroke $\Delta \eta=\eta_{\text {inner }}-\eta_{\text {outer }}$ is shown as a function of body yaw to wing average flapping velocity $\Omega / \omega$. Results are shown for two wings with aspect ratios 4 and 5 in Figure 6.7. Ch was changed by varying the hinge stiffness while keeping the wing geometry and flapping frequency constant $(c=1 \mathrm{cmandf}=100 \mathrm{~Hz})$. The bilateral pitch angle difference in upstroke has the opposite sign of that in downstroke meaning that the outer wing has a larger angle than the inner wing. The magnitude of Passive wing Pitch Asymmetry (PPA) increase linearly with increment of the normalized body yaw velocity, indicating that insects with larger flapping frequency experience less of this effect. The slope of the variations in $\Delta \eta$ with $\Omega / \omega$ is a function of the wing geometry as well as $C h$, implying that the magnitude of PPA could be controlled by adjusting wing kinematics or hinge stiffness during the flight.

The results shown earlier prove that the body motion can cause passive alternations in the wing motion causing asymmetry in the bilateral angles of attack. Asymmetry in the wing kinematics causes asymmetry in the bilateral force production as well. Note that the net velocity of the bilateral wings are also asymmetric which will generate asymmetric forces. This effect exist even in 

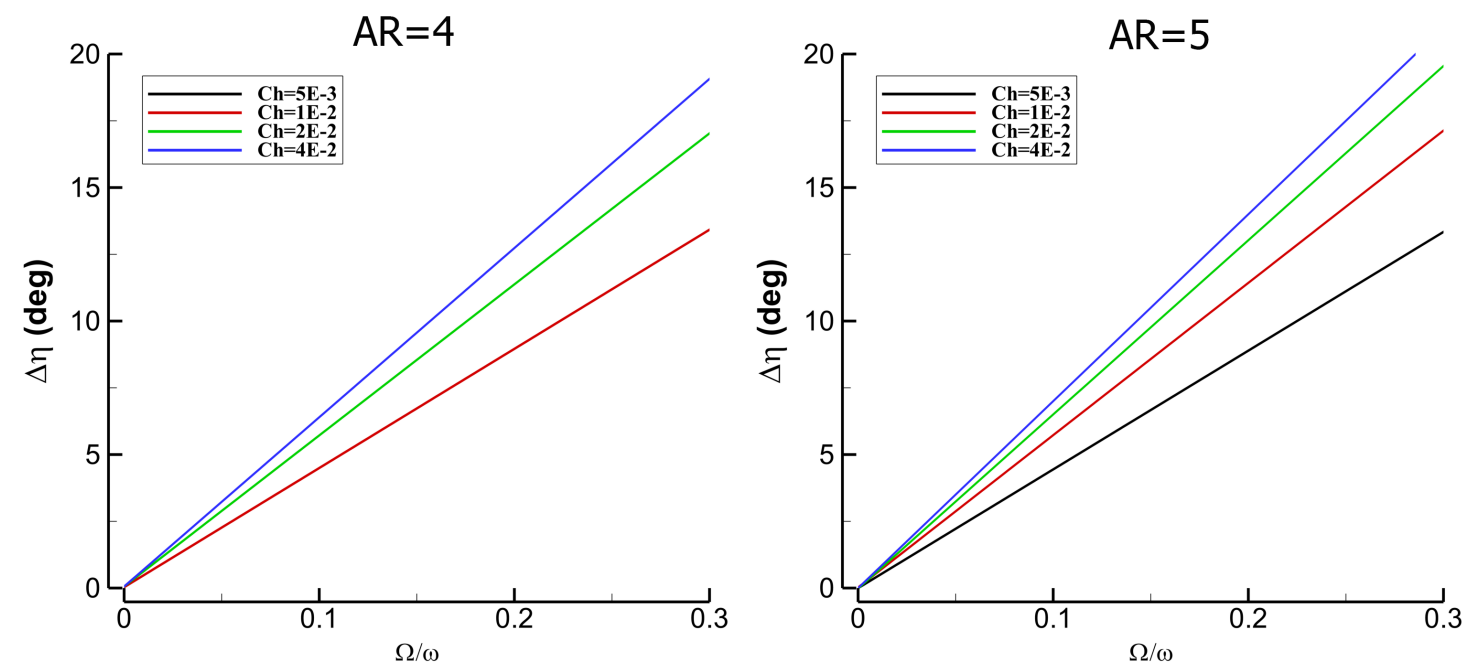

Figure 6.7: Passive bilateral wing pitch difference versus $\Omega_{y a w} / \omega$. The difference between the pitch angle of the inner and the outer wing during downstroke while body is engaged in a yaw turn. Rotation of the body causes asymmetric aerodynamic pressure on the wings which leads to asymmetry in bilateral $C h$ and consequently pitch angles.

the absence of wing-body interactions. Writing the force generated by the wing as the product of the force coefficient and dynamics pressure, the difference in the bilateral forces will be;

$$
\Delta F=0.5 C_{F} \rho_{f} S_{w}\left[(\omega-\Omega)^{2}-(\omega+\Omega)^{2}\right]
$$

Assuming that the bilateral wing kinematics stays symmetric (due to the lack of active or passive modulations of the wings motion), the force coefficient of the bilateral wings remain symmetric. Earlier we showed that the interactions of a flexible wing with the body cause passive modulations of the wing kinematics which leads to asymmetry not only the force magnitude but also in the force coefficient. Figure 6.8 shows the difference in the normal and horizontal force coefficients, $\Delta C_{N}$ and $\Delta C_{H}$ respectively, between the inner and the outer wing during downstroke. In downstroke the force coefficients of the inner wing is larger than that of the outer wing, and in upstroke this relationship is inverse. Results are shown for two wings with aspect ratio 4 and 5 and across a range of $C h$ which are most relevant to the measured values for insects as is presented in Table 6.1. Force coefficients are defined in 6.20. The slope of variations in the force coefficient difference with the normalized body yaw velocity is a function of the wing geometry and $C h$ meaning that the insect can control the magnitude of these variations by altering wing kinematics or the structural stiffness 
of the wing hinge. The variations in the slope with $C h$ are not linear. Table 6.2 lists the slope of the lines in Figure 6.8 and 6.7 for different $C h$ and wing aspect ratio. Also is shown in this table is the slope of the variation of $\Delta \hat{C_{N}}$ and $\Delta \hat{C_{H}}$ defined in Equation 6.23.

$$
\begin{aligned}
\Delta \hat{C}_{N} & =\frac{2 \Delta C_{N}}{C_{N_{\text {inner }}}+C_{N_{\text {outer }}}} \\
\Delta \hat{C}_{H} & =\frac{2 \Delta C_{H}}{C_{H_{\text {inner }}}+C_{H_{\text {outer }}}}
\end{aligned}
$$

Our results show that the horizontal force coefficient of the wing can change up to $30 \%$ of its original value when $\bar{\Omega}$ rises to 0.3 (based on the wing $A R$ and $C h$ ). All the results are shown for downstroke. In upstroke, the slope of the variations of $\Delta \eta, \Delta C_{H}$ and $\Delta C_{N}$ with $\bar{\Omega}$ are the negative of the slopes presented in Table 6.2.

\begin{tabular}{c|cc|cc|cc|cc}
\hline & \multicolumn{2}{|c|}{$C h=5 E-3$} & \multicolumn{2}{c|}{$C h=1 E-2$} & \multicolumn{2}{c|}{$C h=2 E-2$} & \multicolumn{2}{c}{$C h=4 E-2$} \\
\hline & $A R=4$ & $A R=5$ & $A R=4$ & $A R=5$ & $A R=4$ & $A R=5$ & $A R=4$ & $A R=5$ \\
\hline$\Delta \eta($ deg $)$ & 44.6 & 44.4 & 44.6 & 57 & 56.5 & 65.2 & 63.3 & 70.0 \\
$\Delta C_{N}$ & 0.48 & 1.87 & 3.60 & 4.78 & 5.30 & 4.00 & 3.14 & 1.64 \\
$\Delta C_{H}$ & 5.27 & 5.84 & 3.08 & 5.32 & 4.66 & 3.40 & 5.93 & 2.51 \\
$\Delta \hat{C}_{N}$ & 0.25 & 0.72 & 1.18 & 1.46 & 1.58 & 1.51 & 1.30 & 1.00 \\
$\Delta \hat{C}_{H}$ & 1.28 & 1.66 & 1.68 & 2.00 & 1.93 & 2.14 & 2.04 & 2.26 \\
\hline
\end{tabular}

Table 6.2: Slope of the variation of wing pitch and force coefficient with normalized body yaw velocity.

Similar to a yaw rotation, rolling of the body can cause asymmetry in the net motion of the bilateral wings and potentially cause passive wing pitch asymmetries. Figure 6.9 shows the downstrokeaveraged difference in the pitch angle of the inner and the outer wing as a function of $\Omega / \omega$. The body roll velocity $(\Omega)$ was varied systematically for each fixed $C h$ and $A R$. Unlike yaw turns, rolling of the body decreases the pitch angle of the inner wing in both downstroke and upstroke. The magnitude of the asymmetric pitch angle induced by the roll rotations of the body is smaller than that is induced by body yaw (for the same rotational velocity).

The passively induced asymmetry in the bilateral wing pitch angles causes asymmetric force coefficients. Figure 6.10 shows the variations of downstroke-averaged $\Delta C_{N}$ and $\Delta C_{H}$ with $\Omega / \omega$ for two wings with aspect ratio 4 and 5 . Results are presented for four different $C h$ for each wing. In upstroke, the slope of these variations are the same as those in downstroke. This is because body roll has similar effect on altering aerodynamic pressure on the wing in both downstroke and upstroke. 
A
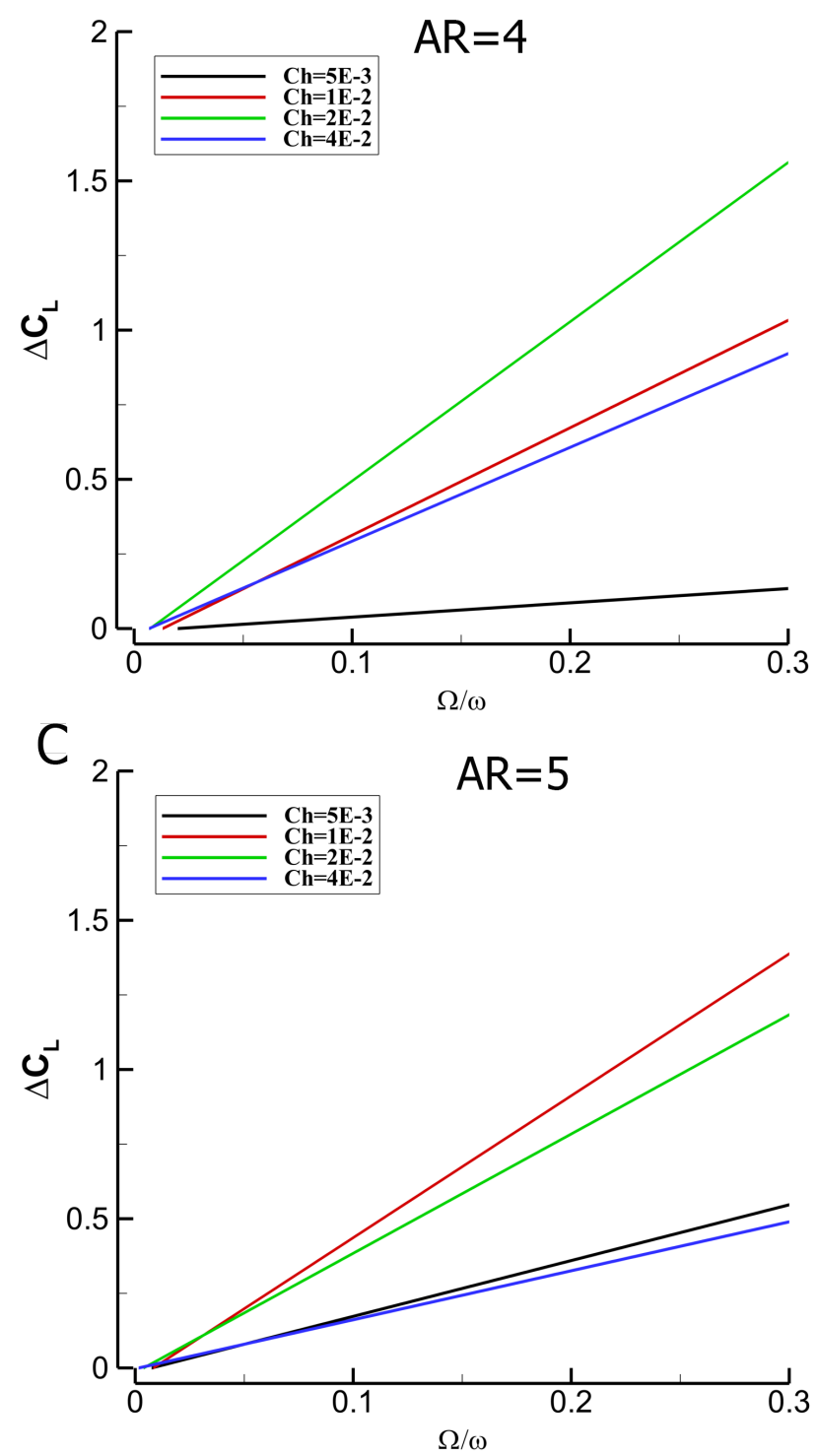

$\mathrm{B}$
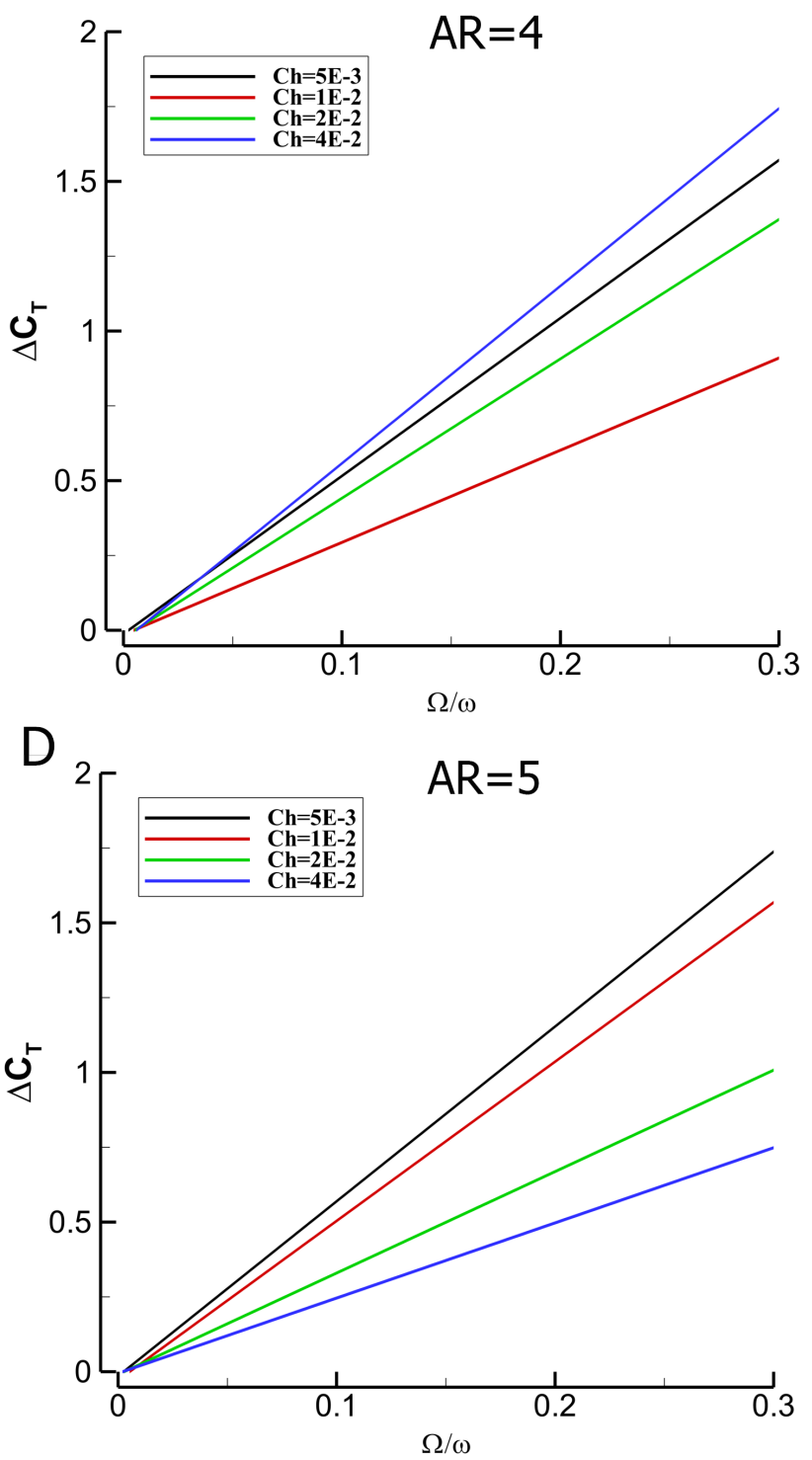

Figure 6.8: Bilateral force coefficient difference versus $\Omega_{y a w} / \omega$. The difference in the force coefficient of the inner and the outer wing during downstroke while body is engaged in a yaw turn. The slops of the line is a function of the wing geometry as well as $C h$. In upstroke, all the slopes are the negative of that in downstroke. 

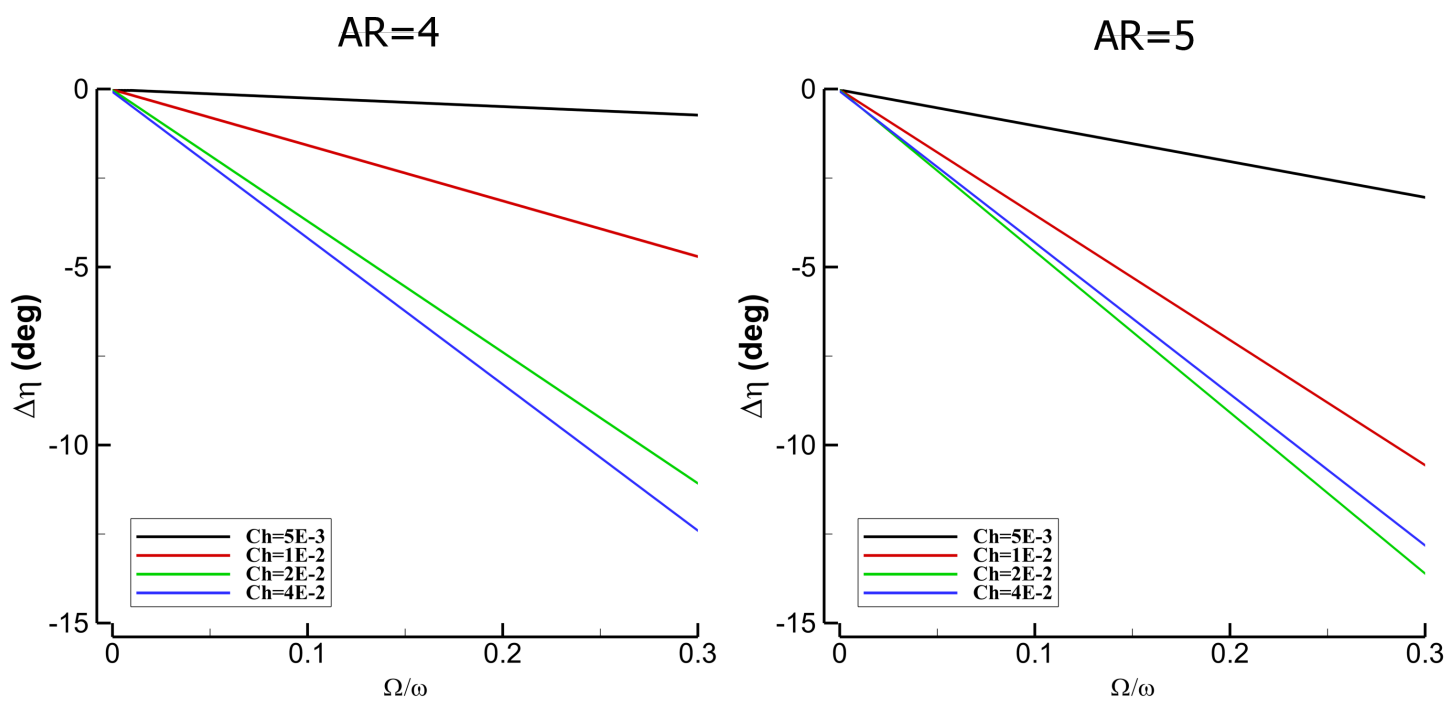

Figure 6.9: Passive bilateral wing pitch difference versus $\Omega_{\text {roll }} / \omega$. The difference between the pitch angle of the inner and the outer wing during downstroke while body is engaged in a roll turn. Rotation of the body causes asymmetric aerodynamic pressure on the wings which leads to asymmetry in bilateral $C h$ and consequently pitch angles. The slope of the lines is a function of the wing geometry as well as $C h$. In upstroke, the slope of the variations is the same as in downstroke.

Table 6.3 lists the slope of the lines in Figure 6.10 and 6.9 for different $C h$ and wing aspect ratio. Also is shown in this table is the slope of the variation of $\Delta \hat{C_{N}}$ and $\Delta \hat{C_{H}}$.

\begin{tabular}{c|cc|cc|cc|cc}
\hline & \multicolumn{2}{|c|}{$C h=5 E-3$} & \multicolumn{2}{c|}{$C h=1 E-2$} & \multicolumn{2}{c|}{$C h=2 E-2$} & \multicolumn{2}{c}{$C h=4 E-2$} \\
\hline & $A R=4$ & $A R=5$ & $A R=4$ & $A R=5$ & $A R=4$ & $A R=5$ & $A R=4$ & $A R=5$ \\
\hline$\Delta \eta($ deg $)$ & -2.39 & -10 & -15.6 & -36.8 & -35.2 & -45.3 & -41.1 & -42.5 \\
$\Delta C_{N}$ & 1.4 & 2.5 & 2.9 & 3.8 & 3.8 & 3.1 & 2.6 & 1.72 \\
$\Delta C_{H}$ & -0.01 & -0.52 & -0.64 & -1.0 & -1.0 & -1.0 & -0.6 & -0.3 \\
$\Delta \hat{C_{N}}$ & 0.76 & 1.0 & 1.0 & 1.25 & 1.19 & 1.2 & 1.12 & 1.08 \\
$\Delta \hat{C_{H}}$ & -0.003 & -0.15 & -0.2 & -0.4 & -0.47 & -0.68 & -0.4 & -0.28 \\
\hline
\end{tabular}

Table 6.3: Slope of the variation of wing pitch and force coefficient with normalized body roll velocity.

\subsection{Conclusions}

Although recent studies have made progress in explaining how the wing motion generates the aerodynamics force [Fry et al., 2003, Ellington, 1984b, Sane and Dickinson, 2002], far less is known about how the wing motion arises and how it's manipulated during aerial maneuvers [Bergou et al., 2010]. Insect wing is driven by the flight muscles in the thorax, but it is also in a constant interaction 

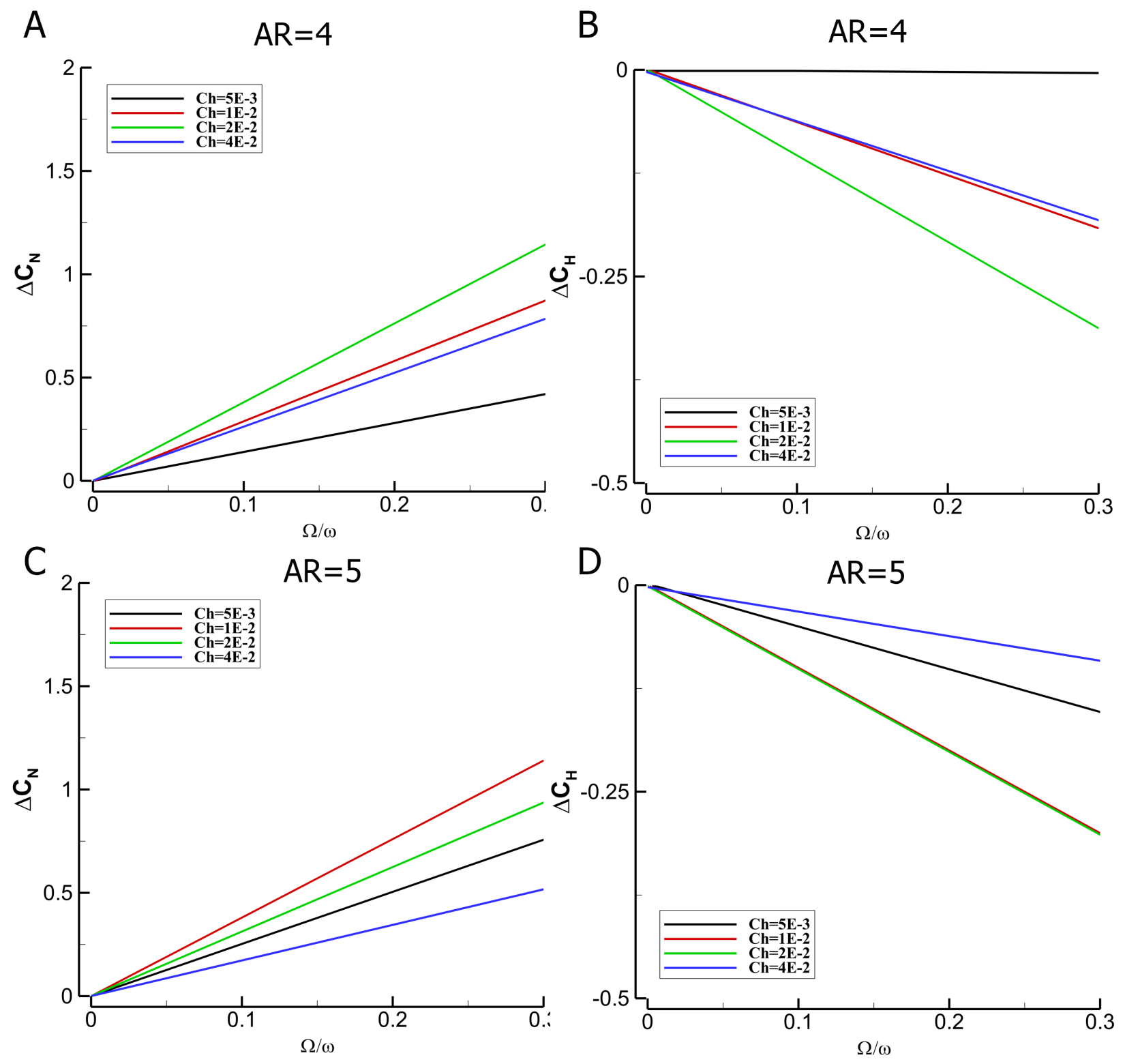

Figure 6.10: Bilateral force coefficient difference versus $\Omega_{\text {roll }} / \omega$. The difference in the force coefficient of the inner and the outer wing during downstroke while body is engaged in a yaw turn. The slops of the line is a function of the wing geometry as well as $C h$. In upstroke, all the slopes are the negative of that in downstroke. 
with the air. In this chapter, we showed that the motion of the wing is governed by the combined effect of several dimensionless quantities including $R e, A R, M, K$ and $C h$. We then characterized the wing dynamics by a systematic analysis on the effect of these parameters. These metrics can be used by engineers to inform design of flapping wings for large lift production and for the control of the wing motion during flight.

We also showed that the motion of the wing is not independent of that of the body. Motion of the body alters the balance between the aerodynamic and elastic force on the wing causing the wing motion to change passively in response to the body motion. The passive variations in the wing kinematics changes the aerodynamic force and therefore the motion of the body. This chain of interactions is the source of coupling in the dynamics of the wings and the body. The strength of the wing-body coupling effect increases as the ratio of the body to wing velocity grows. We particularly showed that lateral rotations of the body (yaw and roll), induce asymmetries in the bilateral wing kinematics as well as the force coefficients. Our results suggest that the conventional model of the insect flight needs to be modify to allow interactions between the wing and the body dynamics. In the next chapter we will show that the modified model predicts the dynamics of the aerial maneuvers of low flapping frequency insects more accurately than the conventional model. 


\section{Chapter 7}

\section{Passive wing Pitch Asymmetry (PPA) in aerial turns of free flying insects}

\subsection{Introduction}

In order to survive, flying insects not only need to stay aloft but also need to steer and maneuver in the air.To execute an aerial maneuver, they finely tune the wing kinematics. Generally, insects use a variety of different kinematics alternations to perform an aerial maneuver such as change in the wing stroke amplitude [Lehmann and Dickinson, 1998], wing pitch angle [Bergou et al., 2010], or speed and timing of wing rotation [Dickinson et al., 1993, Alexander, 1986]. However, there are patterns that are widely common among them. For instance, many insects employ asymmetric pitch angles on the bilateral wings to generate the torque required for executing a yaw turn [Alexander, 1986] [Fry et al., 2003, Bergou et al., 2010, Dickinson et al., 1993]. Flight muscles in the thorax are responsible for implementing the fine adjustments to the wing kinematics [Dickinson, 2003]. Although insects are capable of actively controlling the wing kinematics, the mechanics of the wing and its hinge allows the wing to passively flip in response to the aerodynamic, inertial and the elastic torques. There is a growing body of evidence that supports passive maintenance of the wing angle of attack [Ishihara et al., 2009] as well as the passive wing rotation at stroke reversal [Bergou et al., 2007, Ennos, 1988] in hovering flight. In addition, it was suggested that fruit flies may combine the active and the passive wing kinematic changes in order to perform a turn maneuver 
[Bergou et al., 2010]. Yet, the majority of the previous studies on the wing kinematics control and its association with flight dynamics were focused on high flapping frequency insects such as fruit flies. On the contrary, insects such as damselflies move their wings through the air with a significantly lower velocity. In fact, the neuromuscular structure that drives the wing and controls its motion is fundamentally different for these insects and there is much to be learned about the similarities and differences in the ways these insects use their wings for controlling their body motion.

One of the fundamental differences between the flight dynamics of low and high flapping frequency fliers is that in low flapping frequencies, the time scale of the wing and the body motion are close in magnitude. That is not the case in high flapping frequency flight. Therefore, in the study of high flapping frequency flight, the dynamics of the wings and the body are assumed to be decoupled and the effect of the body motion is only considered on changing the aerodynamic force and torque that acts on the body [Ristroph et al., 2010, Hedrick et al., 2009, Fuller et al., 2014]. Combining a theoretical and experimental approach, here we argue that when the time scale of the wing and the body motions are close, the change in the aerodynamic force and torque not only alters the body motion but also affects the wings. To gain insight into the potential effect that the body motion can impose on the wing motion, we imaged free flight yaw turns of three different species of damselfly (Table 7.1) using three orthogonally oriented high speed cameras. An accurate 3D surface reconstruction technique is then used to extract the three dimensional body and wing kinematics [Koehler et al., 2012]. The extracted flight data reveals that the rotational velocity of the damselfly's body is about 15-30\% of that of its wings. At such speeds, the incoming velocity to the wings changes significantly due to the body rotation and the wing motion is no longer independent from the body motion.

\begin{tabular}{lccccccccc}
\hline & Species & $\begin{array}{c}\text { Body } \\
\text { weight } \\
(\mathrm{mg})\end{array}$ & $\begin{array}{c}\text { Body } \\
\text { length } \\
(\mathrm{mm})\end{array}$ & $\begin{array}{c}\text { Forewing } \\
\text { span } \\
(\mathrm{mm})\end{array}$ & $\begin{array}{c}\text { Hindwing } \\
\text { span } \\
(\mathrm{mm})\end{array}$ & $\begin{array}{c}\text { Forewing } \\
\text { chord } \\
(\mathrm{mm})\end{array}$ & $\begin{array}{c}\text { Hindwing } \\
\text { chord } \\
(\mathrm{mm})\end{array}$ & $\begin{array}{c}\text { Flapping } \\
\text { frequency } \\
(\mathrm{Hz})\end{array}$ & $\begin{array}{c}\text { Flapping } \\
\text { amplitude } \\
(\mathrm{deg})\end{array}$ \\
\hline Damselfly\#1 & $\begin{array}{c}\text { Hetaerina } \\
\text { Americana } \\
\text { Damselfly\#2 }\end{array}$ & 85.0 & 42.0 & 29.0 & 28.0 & 6.0 & 5.5 & 25.0 & 70.0 \\
Dapicalis & 31.0 & 36.0 & 23.0 & 22.0 & 4.0 & 4.0 & 25.0 & 100.0 \\
\hline Ebony & 64.0 & 44.0 & 27.0 & 26.0 & 9.0 & 10.0 & 16.6 & 60.0 \\
\hline
\end{tabular}

Table 7.1: Morphology and kinematics of the three damselfly species in this experiment. All measurements are accurate up to \pm 0.5 measurement unit. 
Damselflies like dragonflies are predators and their survival depends on their ascendancy in maneuverability over other flying insects[Dudley, 2002]. Only a few studies have been conducted toward understanding the flight dynamics of damselfly flight. Those experiments have shown that damselflies accurately modulate their wing flapping amplitude, flapping frequency, inclination of the stroke plane with respect to the flight path, downstroke to upstroke ratio and phase difference between the forewings and the hindwings to control body motion through the air [Ruppell, 1989, Wakeling and Ellington, 1997]. Yet, many aspects of flight dynamics, maneuverability, and wing kinematics control remain unknown in damselfly flight.

\subsection{Damselfly's yaw turn dynamics}

The yaw turn amplitude is between 160 to 170 degrees for all three damselflies. In spite of the differences in the morphology and kinematics, all turns were completed in about 5 wingbeats. All maneuvers are very close to a pure yaw turn. The variations in the roll and pitch angles are little when compared to the yaw angle. The body and wing kinematics as well as the motion reconstruction of the damselfly $\# 1$ are shown in Figures 7.1 and 7.2. Consistently for all three damselflies, the body yaw velocity rises during the first two wingbeats. This phase is referred to as acceleration phase in this work (Figure 7.2). About 30\% of the body heading change takes place within this phase. We did not observe a period of relatively constant angular speed in these maneuvers, as was previously observed in other flying insects such as fruit flies [Dickinson et al., 1993]. The angular velocity of the body then started to drop slowly. This phase, which is referred to as the deceleration phase, lasts for about 3 wingbeats. The majority of the heading change takes place during this phase. The maximum yaw velocity as well as the flapping frequency varies between the species (Table 7.2). As the yaw velocity increases and decreases during the two phases of the maneuver, the half-strokeaveraged (averaged over a downstroke or an upstroke) ratio of the body to the wing angular velocity, , remains at about 0.15-0.3 (except at the first and last half-strokes of the maneuver when the body rotational velocity is very low).

Figure 7.2 shows the wing kinematics of damselfly $\# 1$. The wing flapping angle represents the angle that the wing travels in the stroke plane. The stroke plane is defined as the mean square plane of the position of the wing's marker points within each downstroke or upstroke. The wing 


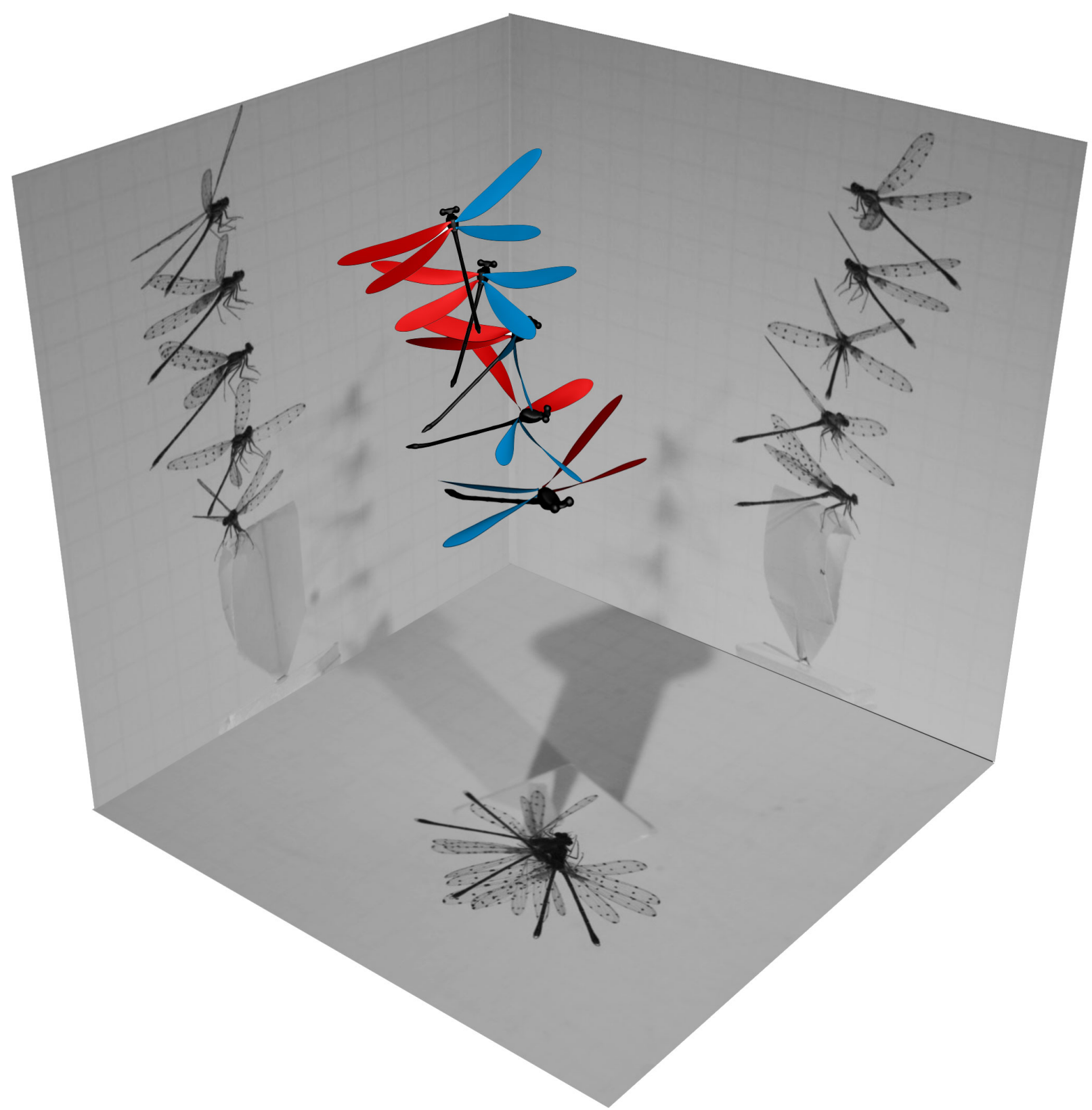

Figure 7.1: Motion reconstruction of the $\mathbf{1 6 0}$ degree turn of damselfly \#1. A selected sequence of images of the reconstructed wing and body are superimposed on high speed images for damselfly $\# 1$. 


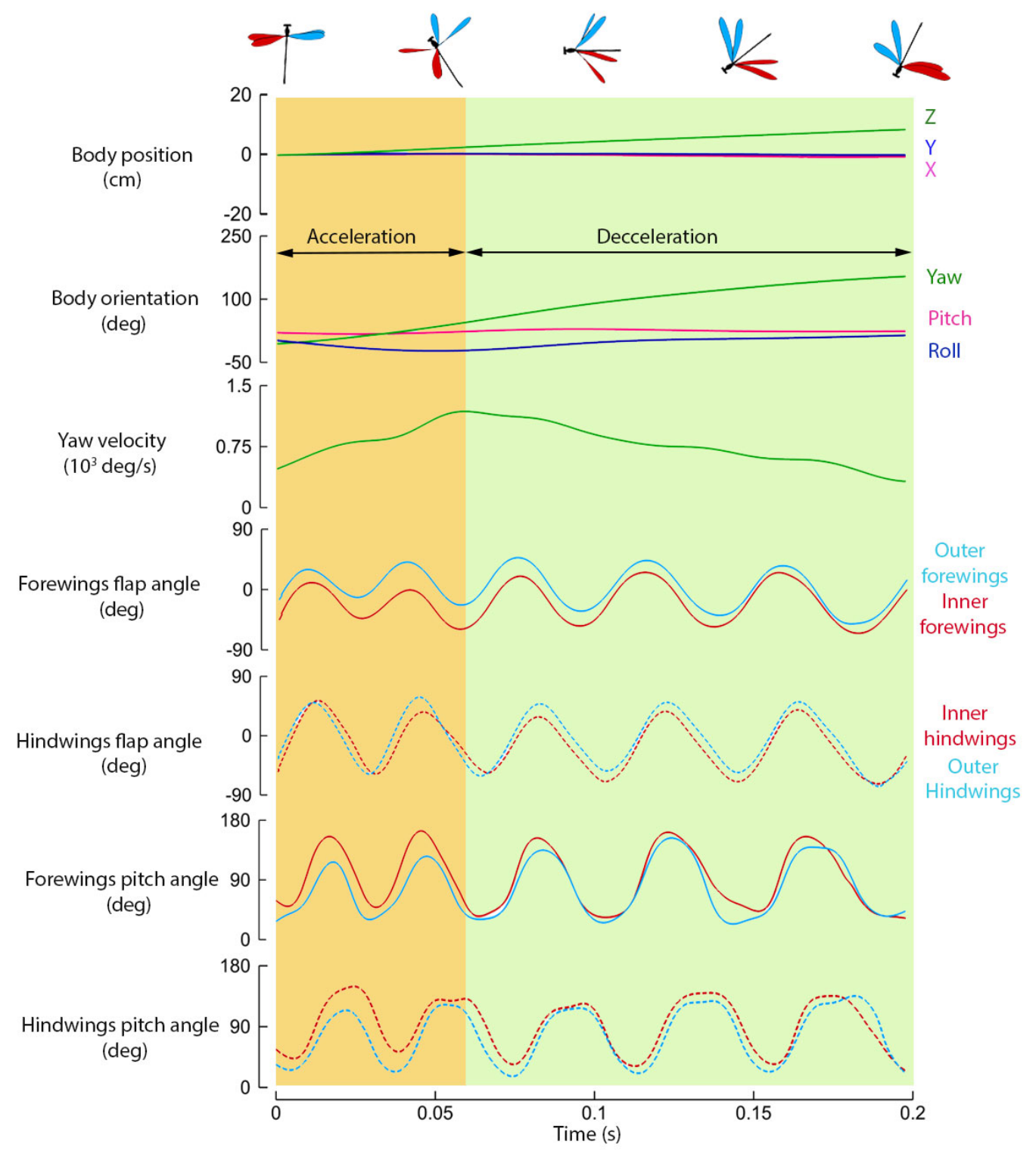

Figure 7.2: Wing and body kinematics of damselfly \#1. The body position and orientation as well as the wing flap and pitch angles. The reference frame for the body orientation measurement is parallel to the initial orientation of the insect and therefore initially all there Euler angles are zero. The flap angle is the angle that wing travels in the stroke plane and pitch angle is the angle between the wing's mid chord line and the stroke plane. The accuracy of the body yaw, pitch and roll angle measurements are $\pm 1, \pm 2, \pm 5 \mathrm{deg}$. The wing flapping angle is accurate up to $\pm 1 \mathrm{deg}$. The accuracy of the wing pitch angle measurements is $\pm 2 \mathrm{deg}$. 


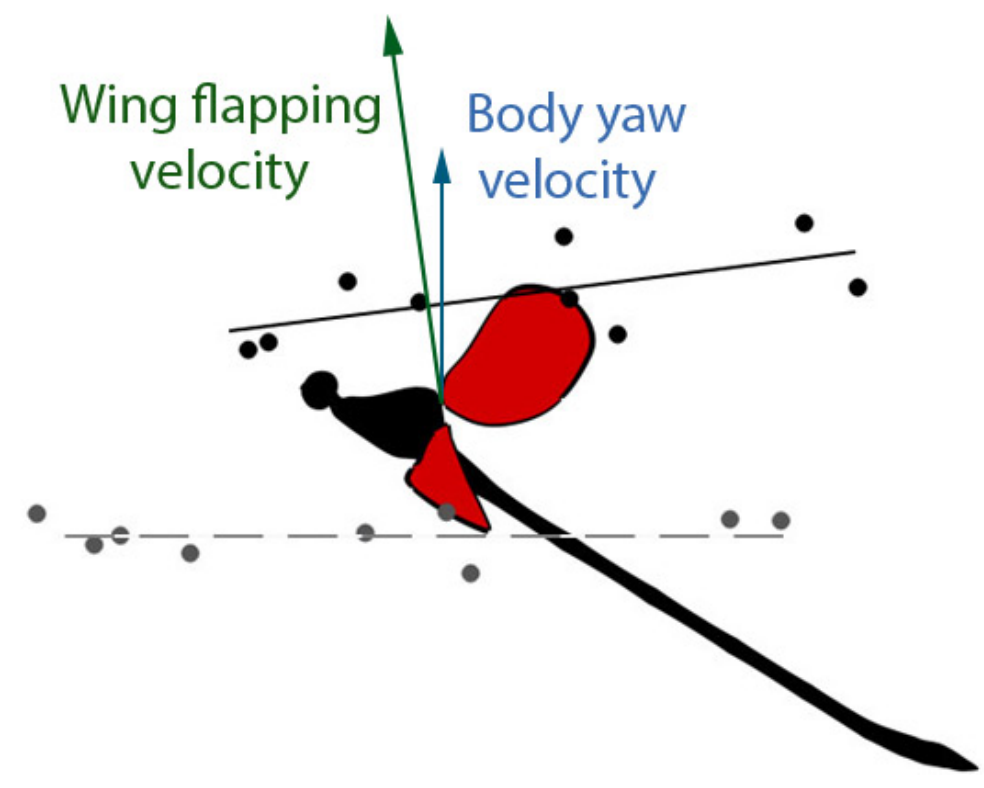

Figure 7.3: Stroke plane in damselfly yaw turns The average stroke plane of the forewings and hindwings are shown. The dark green and dark blue vectors represent wing flapping and body angular velocity. Because of the inclination of the stoke plane, there is a slight misalignment between these two vectors.

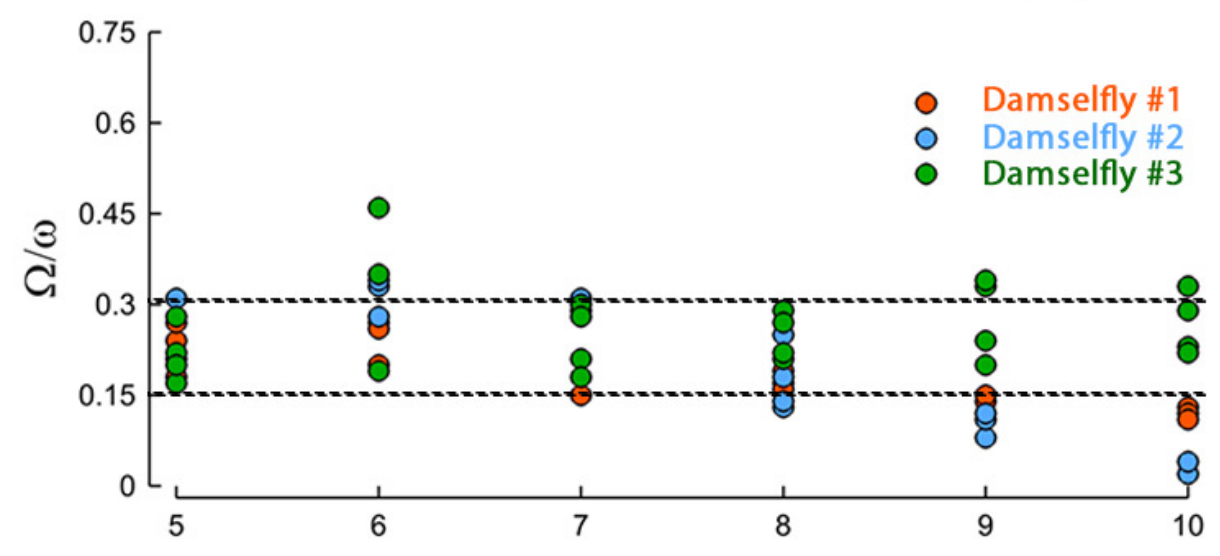

Figure 7.4: Ratio of the average body to wing angular velocity in damselfly yaw turn. Ratio of the average body to wing angular velocity within the 6 half-strokes of the deceleration phase for all three damselflies. This value remains within a tight band during the most of the maneuver duration. 


\begin{tabular}{lcccc}
\hline & $\begin{array}{c}\text { Turn } \\
\text { amplitude } \\
(\mathrm{deg})\end{array}$ & $\begin{array}{c}\text { Maneuver } \\
\text { duration } \\
(\mathrm{ms})\end{array}$ & $\begin{array}{c}\text { Maneuver } \\
\text { duration } \\
\text { (wingbeats })\end{array}$ & $\begin{array}{c}\text { Max yaw } \\
\text { velocity } \\
\left(10^{3} \mathrm{deg} / \mathrm{s}\right)\end{array}$ \\
\hline Damselfly\#1 & 160 & 200 & 5.5 & 1.2 \\
Damselfly\#2 & 170 & 180 & 5.0 & 1.7 \\
Damselfly\#3 & 170 & 300 & 5.0 & 0.7 \\
\hline
\end{tabular}

Table 7.2: Kinematics of the body yaw maneuver.

pitch is the angle between the wing mid chordline and the stroke plane. To execute a yaw turn, in downstroke, the damselfly flaps the inner wings with a higher pitch angle compared to the outer wings. That causes the drag to increase on the inner wings and turn the body. In upstroke, thrust increases on the outer wings due to its higher wing pitch angle which causes the body to turn in the same direction. The high magnitude of the bilateral wing pitch asymmetry within the first two wingbeats causes the body rotational velocity to rise rapidly. The magnitude of the wing pitch asymmetry drops for the deceleration phase. It was previously shown that the decelerating and stopping the turn does not require significant help from the active control. The asymmetric variation in the incoming velocity of the bilateral wings causes a strong damping effect known as Flapping Counter Torque (FCT) which is expected to quickly dampen the angular velocity of the damselfly body [Hedrick et al., 2009]. FCT predicts the yaw velocity of the damselfly to fall to half its value in less than a wingbeat. However, this is not consistent with our observation. All the damselflies in our experiment were able to maintain the body velocity for a significantly longer time.

To probe the dynamics of the deceleration phase, we investigated the association between the wing and body motion. Our measurements and analysis show that the half-stroke-averaged wing pitch angles as well as the bilateral pitch angle difference are independent from the body angular velocity. In most insects, the central control system (which controls the wing motion) receives feedback from the body motion [Deng et al., 2006]. Therefore, the lack of correlation between the body motion and the wing pitch angle suggests that the measured wing pitch values are not dominantly forced by the active control. In addition, the relationship between the wing pitch angle and the flapping velocity is somewhat random (Table 3). Figure 7.5 shows the half-stroke-averaged wing pitch angle versus the corrected wing angular velocity. A strong correlation is found between these two parameters for both forewings and hindwings of the damselflies. The corrected wing angular velocity is defined as the flapping velocity of the wing, with respect to the body, corrected 
by the rotational velocity of the body. For instance, in downstroke, the corrected angular velocity of the outer wing is the sum of the flapping velocity and the body yaw velocity. This for the inner wing (in downstroke) is the flapping velocity of the wing minus the yaw velocity of the body. The vector of the flapping velocity of the wing is slightly tilted with respect to the vector of the body yaw velocity (Figure 7.3). This is because of the inclination of the wing stroke planes. For the damselfly $\# 1$, the stroke plane angles of the forewings and hindwings (measured with respect to a line which connects the head and tail of the body) vary between 42 to 49 degrees and 23 to 40 degrees, respectively. The body pitch angle remains at about 28 deg throughout the maneuver. Therefore there is less than19 deg misalignment between the vector of the body yaw velocity and the forewing flapping velocity. This magnitude is even lower for the hindwings. Also, the center of mass ascends about 1-2 body lengths, in all three maneuvers, while the insect is engaged in a yaw rotation. The magnitude of the average ascending velocity is less than $0.02 \%$ of the average translational velocity of the wingtip due to its flapping motion. Thus, we assumed it has negligible effect on the wing dynamics. The slope of the regression line between the half-stroke-average wing pitch angle and the corrected angular velocity of the wings is very close for the forewings and the hindwings but the hindwings move through the stroke plane with a higher pitch angle. Due to the differences in the morphology and kinematics, this slope varies among the species as well. Although the statistical correlation observed in the flight data does not necessarily imply causation, it suggests existence of a relationship between the wing pitch angle and the corrected wing angular velocity. To prove that the rotation of the body and the consequent change in the incoming velocity to the wings has in fact caused the variations in the wing pitch angles, we construct a physics-based model of the wing pitching and show that corrected wing angular velocity can successfully predict the measured wing pitch angles.

\subsection{Response of the physics-based model of the wing to the body rotation}

To reveal the underlying physics behind the relationship between the wing pitch angle and the corrected wing velocity by the body rotation we construct a physics-based model of the damselfly wing. Insect wings are flexible and deform under the action of the aerodynamics and inertial 

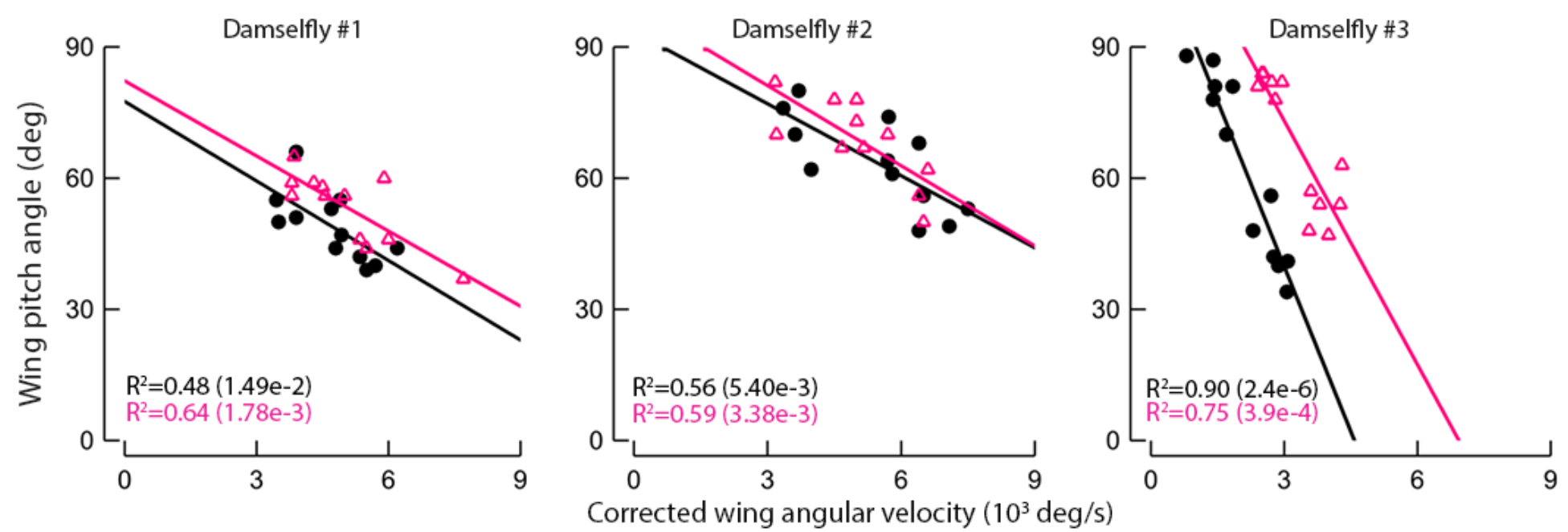

Figure 7.5: Half-stroke-averaged wing pitch angle versus the corrected wing angular velocity during the deceleration phase. The black and deep pink, respectively, represent the forewings and hindwing. On each graph, the correlation coefficients, R2, are shown with the same color as the respective regression line. P-values are shown in the brackets. The difference in the least square regression slop suggests difference in Ch for these insects.

forces [Daniel and Combes, 2002, Ennos, 1988]. However, for simplicity, we modeled the wing as a rigid wing with an elastic pitching hinge [Ishihara et al., 2009, Bergou et al., 2010, Ennos, 1988, Whitney and Wood, 2010]. The flapping motion of the wing through the stroke plane is prescribed. The dynamics of the wing pitching is governed by the Equation 7.1 below. The governing equation of the pitching dynamics is solved in a frame attached to the wing which its $\mathrm{x}$-axis is aligned by the wing's rotation axis (Fig. S1A).

$$
I_{x x} \dot{\omega}_{x}+I_{x y}\left(\dot{\omega}_{y}-\omega_{x} \omega_{z}\right)=\tau_{\text {aero }}+\tau_{\text {elastic }}
$$

$\left(\omega_{x}, \omega_{y}, \omega_{z}\right)$ is the vector of the angular velocity of the wing in the wing coordinate system. $\mathrm{x}$-axis of the wing passes through the rotation axis. y-axis points forward and z-axis is in the direction of vector product of $\mathrm{x}$ and $\mathrm{y}$ axes. $\tau_{\text {aero }}$ and $\tau_{\text {elastic }}$, respectively, are the aerodynamic and elastic reaction torques about the axis of rotation of the wing. $I_{i j}$ is the tensor of moment of inertia of the wing in the wing coordinate system. It is calculated assuming homogeneous mass distribution across the wing area. The elastic torque about the wing axis of rotation is linearly proportional to the wing deflection. The pitch angle of the wing is determined by the balance between the inertial, aerodynamic and reaction elastic forces, with inertial forces playing a less important role 


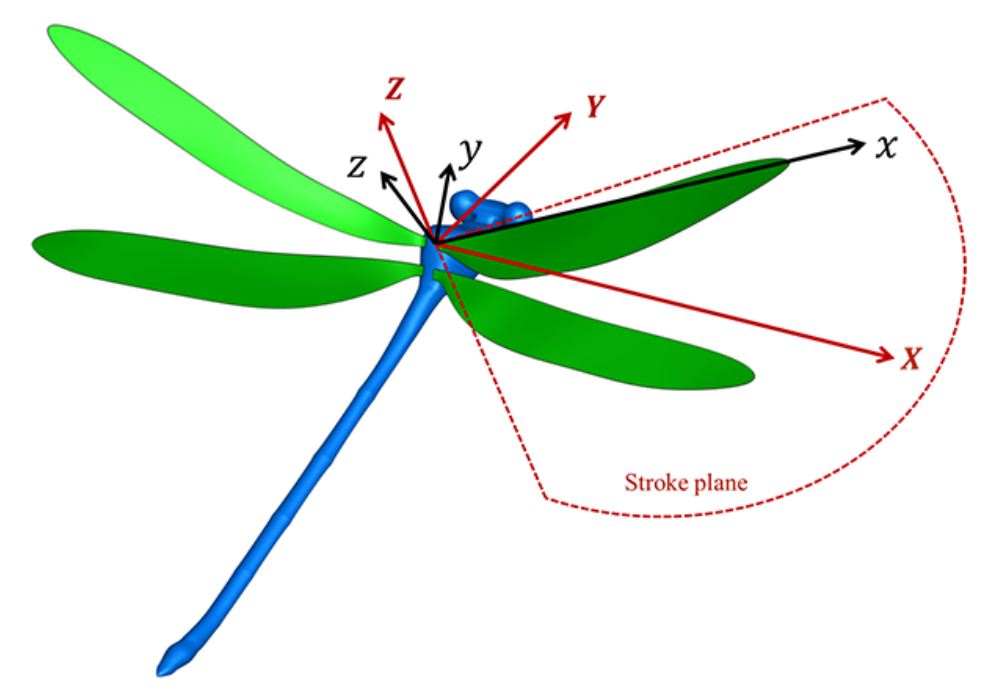

Figure 7.6: Coordinate systems of the wing and the body.

[Ishihara et al., 2009]. The elastic torque about the wing rotation axis is modeled by a linear torsion spring (Equation 7.2).

$$
\tau_{\text {elastic }}=-G\left(\psi-\psi_{0}\right)
$$

$\psi_{0}$ is the spring rest angle and was set to $90^{\circ}$, showing the tendency to keep the wing vertical. $C h$ number defines the relative ratio of the fluid pressure to the elastic force (9).

$$
C h=\frac{\rho_{f} V_{w} \bar{c}^{4} f}{G}
$$

$\rho_{f}$ is the fluid density. $\bar{c}$ and $f$ are wing average chord and flapping frequency, respectively. $V_{w}$ is the average translational velocity of the wing tip.

When an insect experiences a whole body rotation, in downstroke (upstroke), the net incoming velocity increases on the outer (inner) wing and it decreases on the inner (outer) wing. This results in asymmetric variation in the fluid dynamic pressure on the wings. Since, in the absence of active control, the elastic properties of the wing hinge remain unchanged, the relative strength of the fluid 
force (and torque) compared to the elastic reaction force, $C h$ number, varies. The asymmetric change in $C h$ number then causes an asymmetric response of the wings' pitch angles. For instance, on the outer wing during downstroke, the fluid forces are strengthened (since the center of pressure of the wing is located behind the axis of rotation) which results in a stronger pitch down torque being exerted on the wing. Similarly, on the inner wing, the fluid forces are weakened and elastic forces are able to rotate the wing further upward which causes a higher pitch angle on this wing (Figure 7.7). This is consistent with the experimental relationship we have observed between the corrected wing angular velocity and the wing pitch angle (in Figure 7.5). However, the effectiveness of this phenomenon depends on the extent to which the $C h$ number changes due to the body rotation. The relative change in the $C h$ number of the bilateral wings depends on the relative magnitude of the angular velocity of the body compared to that of the wing.

$$
C h_{\text {maneuver }}=\frac{\rho_{f} L_{w} \bar{c}^{4} f}{G} \omega\left(1 \pm \frac{\Omega}{\omega}\right)
$$

For a damselfly in a fast yaw turn, $C h$ of the wings may vary up to $35 \%$ compared to its value when the body rotational velocity is zero. Thus, we hypothesize that the rotation of the body and the consequent asymmetric change in $C h$ number of the bilateral wings causes asymmetry in the dynamics of the bilateral wings.

To test this hypothesis, we conduct a numerical experiment. In this experiment, the pitch angles of the bilateral wings are numerically calculated by integrating Equation 7.1. The geometry, the flapping kinematics and the hinge torsional stiffness of the bilateral wings are kept identical. The rotation of the body is prescribed by a second order polynomial with a peak value of $1500 \mathrm{deg} / \mathrm{s}$ (Figure 7.8). At the beginning of the maneuver, the two wings have an identical pitch angle. As the body angular velocity increases, the pitch angles of the wings start to depart from each other. During each downstroke, the fluid velocity on the outer wing increases and it decreases on the inner wing. That causes the average pitch angle of the outer wing to drop and that of the inner wing to rise. Similarly, during upstroke, the average pitch angle of the outer wing is enhanced and that of the inner wing is reduced. We termed this phenomenon as Passive wing Pitch Asymmetry (PPA) because the alternations in the wing kinematics are not actively forced by the flight muscles in the thorax but are passively induced by the body rotation. The passive variations in the wing pitch 

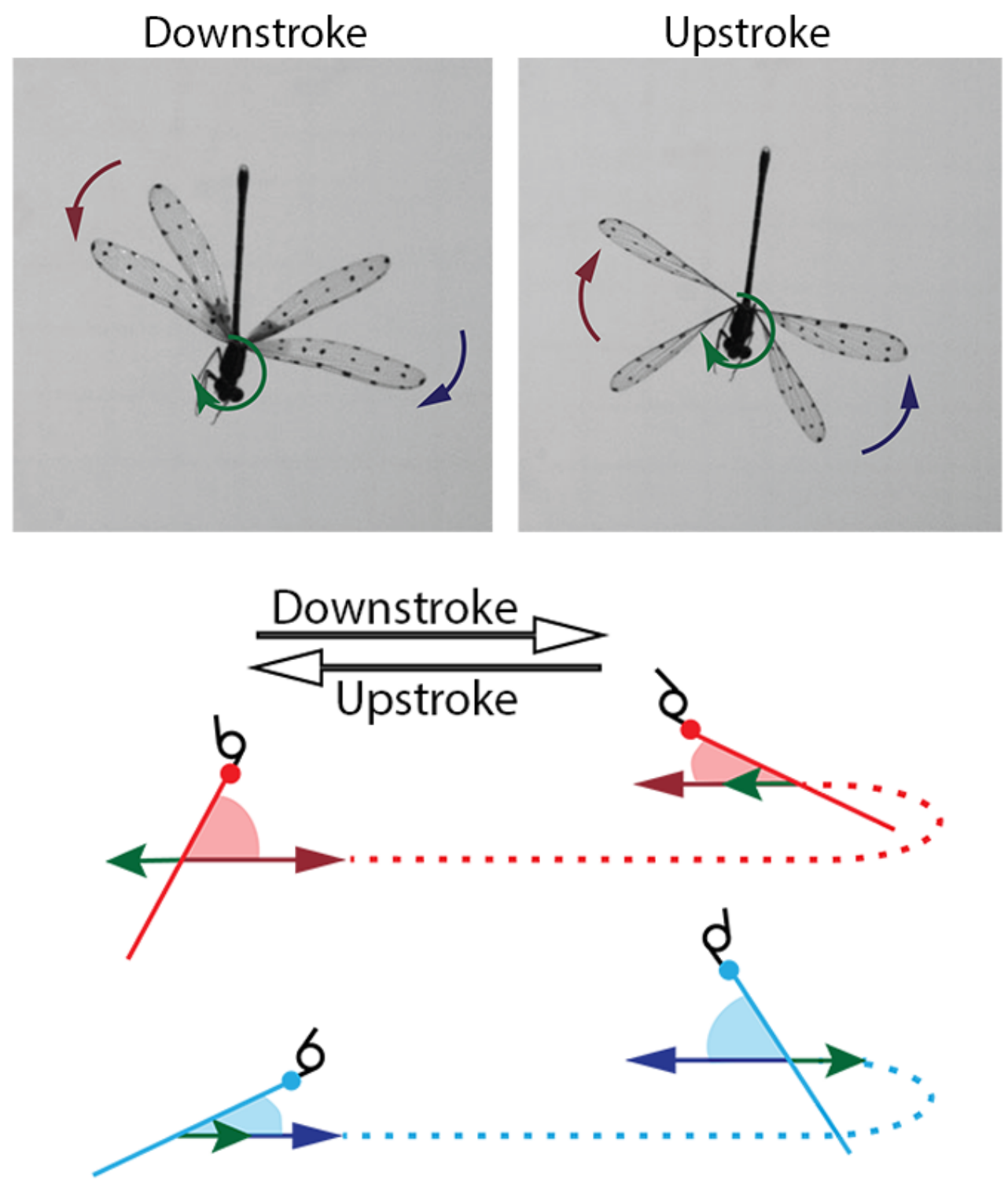

Figure 7.7: Wing and body velocity in Damselfly in yaw turn. The angular velocity of the body enhances the velocity of the outer wing in downstroke and reduces it in upstroke and vice versa for the inner wing. Increasing or decreasing the velocity changes the balance between the aerodynamics and elastic torque and consequently causes asymmetric alternation in the bilateral wing pitch angles. The outer and inner wings are shown by blue and red, respectively. 
angles are in such way that it helps maintain the yaw velocity of the body.

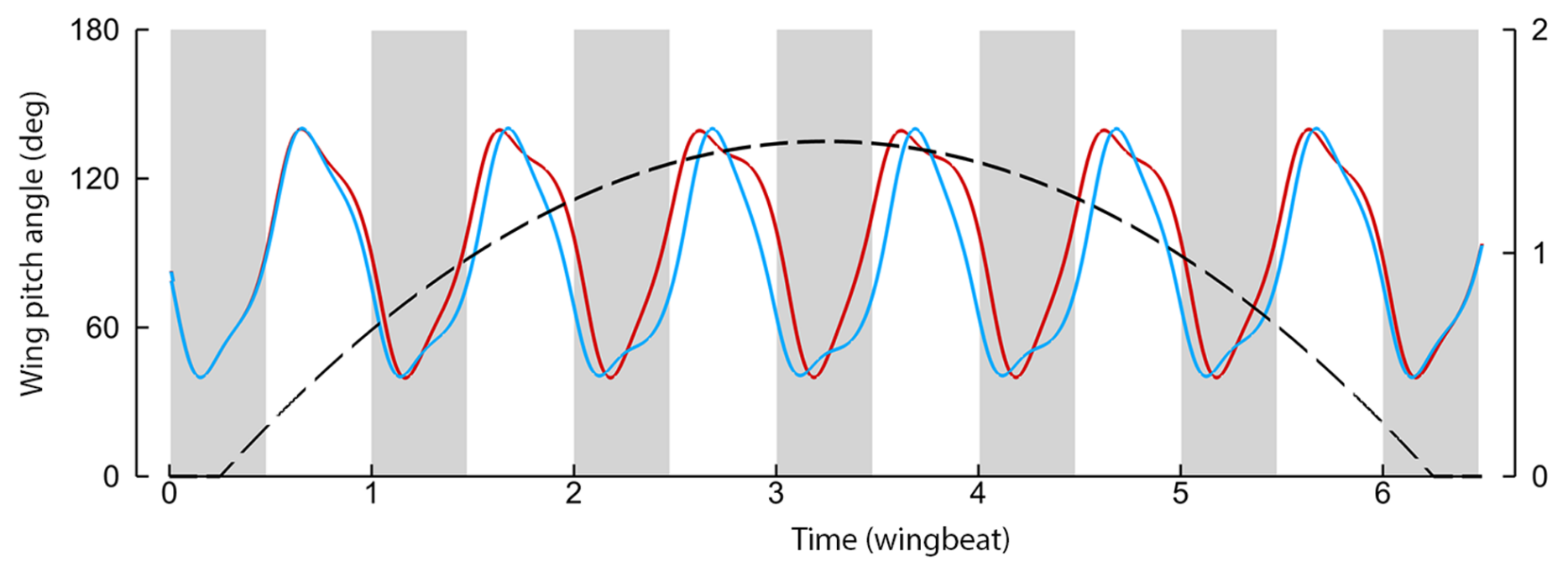

Figure 7.8: Passive wing pitch asymmetry during a model turn maneuver. Stroke by stroke augmentation of the passive wing pitch asymmetry during a model turn maneuver. The red and blue lines represent the pitch angles of the inner and outer wings, respectively. The black line shows the time course of the prescribed body angular velocity which is shown on the right vertical axis. The body turn velocity is prescribed by a second order polynomial. Downstrokes are shaded gray.

The magnitude of the bilateral wing pitch difference increases by increase in the body rotational velocity. As the body accelerates and the velocity increases, the damping effect grows accordingly and an insect requires more active torque to maintain its turning velocity [Hedrick et al., 2009]. Our results suggest that even if the active modulation of the wing kinematics stays uniform throughout the maneuver, this passive mechanism will augment the asymmetry in a similar fashion to what was observed in the insect maneuvers [Fry et al., 2003, Wang et al., 2003]. Although active tuning of the wing kinematics is necessary for the initiation of the maneuver, this passive mechanism helps maintain and even enhance the wing kinematics asymmetry. In addition, at the transition from downstroke to upstroke, the inner wing's rotation time was advanced compared to the outer wing (and vice versa in upstroke). The change in wing rotation time is also a consequence of the shift in the balance between the aerodynamic and the elastic torque. This implies that the same mechanism is responsible for the variations in the average wing pitch angle and the wing rotation timing at the stroke reversal. Thus, an insect does not necessarily control these parameters independently. 


\subsection{Estimation of the damselfly Wing hinge stiffness}

We were not able to directly measure the wing hinge stiffness but that value can be calculated once the wing and body kinematics, wing geometric parameters and the wing pitch angles are measured. Our model predicts that the slope of the variations of the wing pitch angle versus the corrected wing velocity is a function of $C h$ and thus the hinge stiffness. Note that $G$ is the only free parameter once the wing geometry and kinematics were measured. Changing the body rotational velocity (in steps of $250 \mathrm{deg} / \mathrm{s}$ ) we calculate the average wing pitch angle and plot the results. We then vary $\mathrm{G}$ and repeat the same calculation. This plot is shown for a forewing of damselfly $\# 3$ if Fig. S1B. As was expected, by decreasing G (and therefore increasing $C h$ ), the slope of the variation of the wing pitch angle with the corrected wing velocity increases. No attempt was made to find the exact value of $\mathrm{G}$. Yet, the $\mathrm{G}$ value that gives the closest slope to the experimental flight data (Fig. 2 in the main text) was used for the wing pitch angle prediction. The fact that the $\mathrm{G}$ value attained this way gives an accurate prediction of the wing pitch angle further proves the consistency of our theory with the observed flight behavior.

\subsection{PPA in damselfly yaw turn}

Figure 7.10 shows the predicted versus the measured half-stroke-averaged wing pitch angle for all three insects (during the deceleration phase). In calculation of the wing pitch angle we matched the half-stroke averaged flapping velocity and body yaw velocity to the experimentally measured values. Note that in Figure 7.10 the hinge stiffness of the fore and hind wings is not necessarily the same. However, we assume that the right and the left wings (of each pair) have identical values. In addition, this value remains constant throughout the deceleration phase (assuming there is no active control). The predicted pitch angle values are in excellent agreement with the measurement regardless of the difference in the morphology and kinematics of the wings and the body among species. Therefore, we infer that the rotation of the damselfly's body induces passive alternations in the wings pitch angles by shifting the balance between the aerodynamics and the elastic torque exerted about the wing's rotation axis. This variation is bilaterally asymmetric and increases due to an increase in the body rotational velocity. The asymmetric variation in the bilateral wing pitch angles arises from the difference in the velocity of the outer and the inner wing and therefore is 


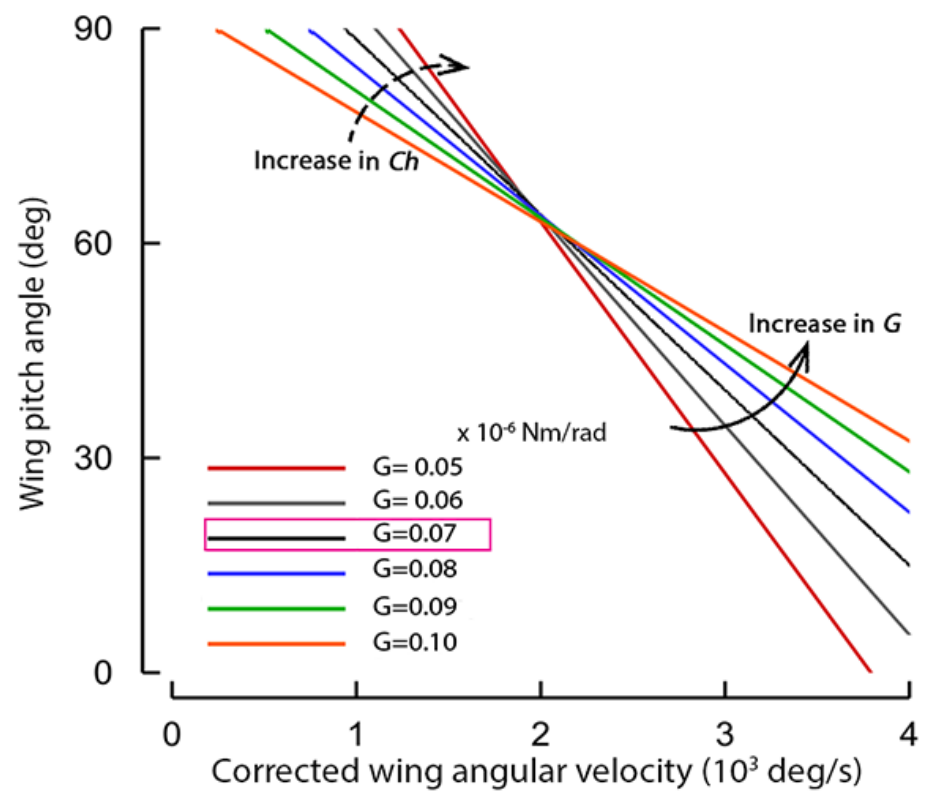

Figure 7.9: Estimation of the damselfly wing hinge stiffness. The wing pitch angle versus the corrected wing velocity for a wing with the geometry and kinematics of the damselfly \#3. The hinge stiffness was varied until the slope of the relationship resembles that of the experimental observation. This was achieved for $\mathrm{G}=0.07 \mu \mathrm{Nm} / \mathrm{rad}$, the theoretical and experimental slopes are 24.49 and 25.02, respectively. Similar procedure was performed for forewings and hindwings of each damselfly. 
restricted to flapping flight. As a result a damselfly is capable of maintaining its body rotational velocity for a longer time (after the initial acceleration phase) even if no further active control is employed.

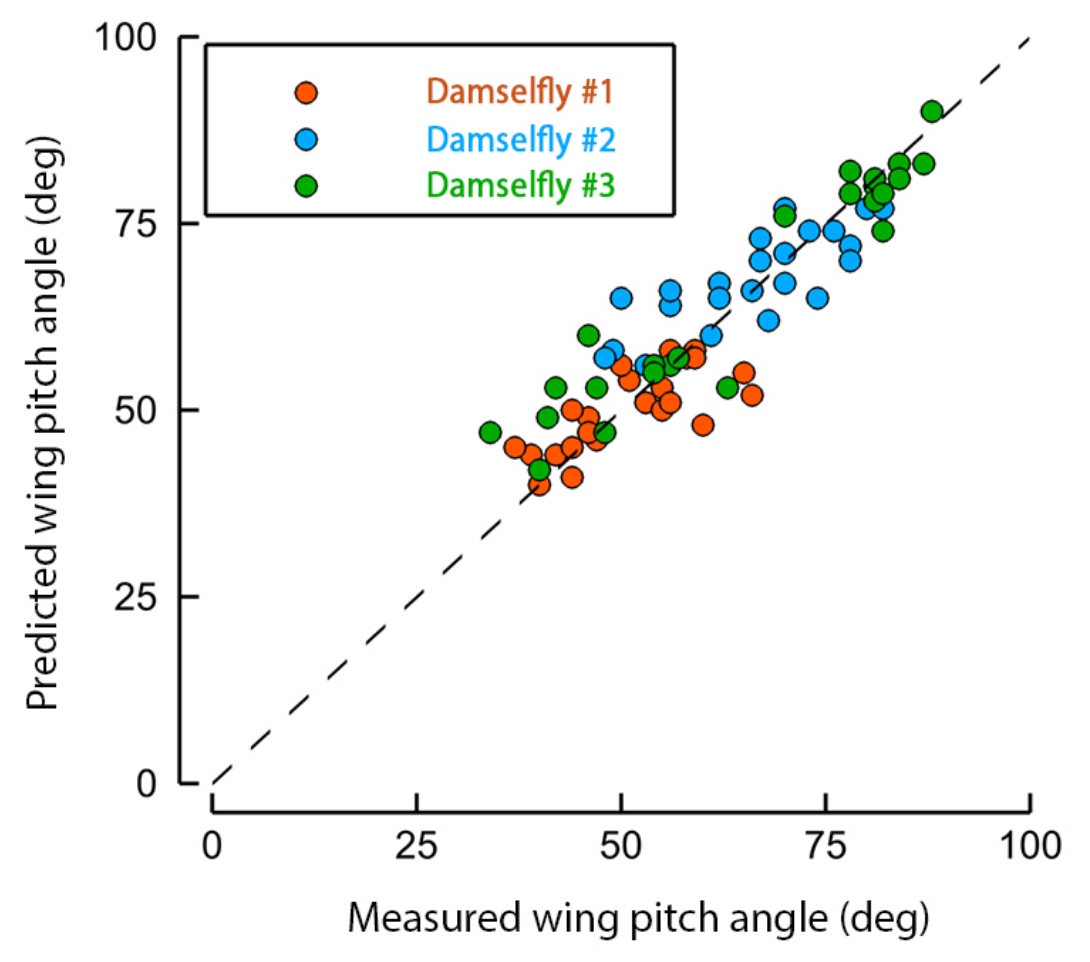

Figure 7.10: Prediction of the wing pitch in damselfly yaw turn. Predicted versus the measured wing pitch angle for all three damselflies. The predicted value is calculated by matching the half-stroke-averaged wing flapping velocity as well as the body yaw velocity to the experimentally measured values.

\subsection{Force and torque due to the PPA}

The aerodynamic force on the wings is commonly described by the product of the force coefficient and the dynamic pressure. The asymmetric change in the dynamic pressure of the bilateral wings (due to the body rotation) was previously shown to induce a passive damping torque on the body, FCT. Our results suggest that the passive change in the wing pitch angle of the bilateral wings causes the force coefficient of the wings to alter as well. In this section, we show that the FCT alone does not adequately predict the yaw velocity half-life of the damselfly and including the effect of the 
PPA-induced force coefficient asymmetry is essential for the accurate description of the dynamics of the damselfly's yaw turn.

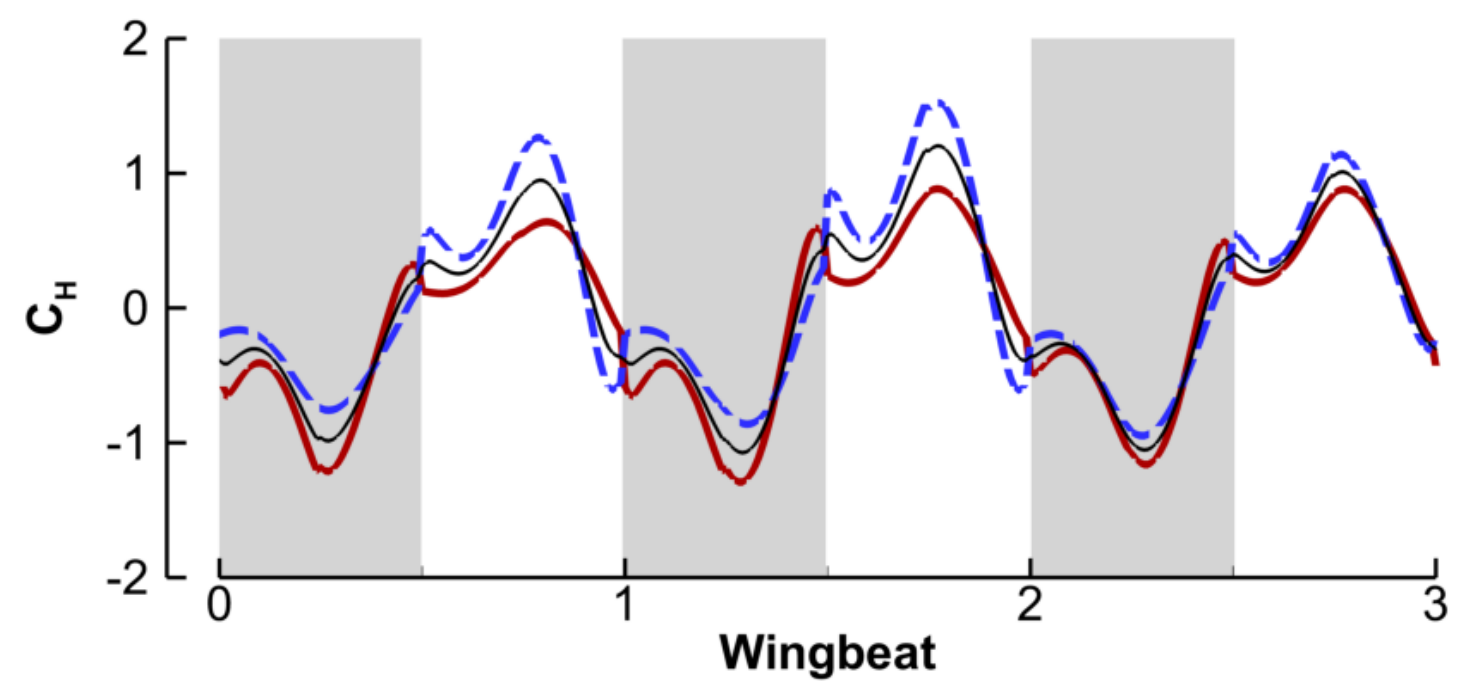

Figure 7.11: Horizontal force coefficient asymmetry. Stroke by stroke augmentation of the passive horizontal force coefficient asymmetry of the damselfly forewings during the turn maneuver. The red and blue lines represent the inner and outer wing, respectively .Solid black line shows the average force coefficient, $C_{0}$.

Figure 7.11 shows the horizontal force coefficient, $C_{H}$, of the inner and the outer forewings of the damselfly during the phase-II. In this simulation we matched the half-stroke-averaged wing flapping velocity and body yaw velocity to the measured values. Note that, in the calculation of $C_{H}$, the wing's reference velocity is different in downstroke and upstroke as well as for the inner and the outer wings. This is consistent with the physics of the phenomenon. Similar definition for the force coefficient of a flapping wing was previously used to separate the effect of the kinematics from the non-kinematics terms [Hedrick et al., 2009]. A jump in the magnitude of $C_{H}$ in the transition from the downstroke to the upstroke is therefore observed. The difference between the inner and the outer wing, on the other hand, is a result of the asymmetric wing pitch angles which causes $C_{H}$ of the inner wing to increase during the downstroke and that of the outer wing to decrease. Similarly, in upstroke, the outer wing has a higher force coefficient compared to the inner wing. This can be 
better understood by noting that if the wing kinematics (flapping as well as the pitching) of the bilateral wings were symmetric, the alternation in the horizontal force would be only due to the change in the incoming velocity (dynamic pressure) and dividing the force by the dynamic pressure gives identical values for both wings. The enhancement in the force coefficient of the wing with low incoming velocity and its reduction on the wing with high incoming velocity results in lessening the effect of the damping due to FCT. That allows the insect to maintain its angular velocity for a longer time without additional active control.

Assuming symmetric flapping, in downstroke, the half-stroke-averaged force coefficient of the inner wing increases and that of the outer wing decreases in such a way that,

$$
\begin{aligned}
& \bar{C}_{H_{\text {inner }}}=C_{0}+\Delta \bar{C}_{H} / 2 \\
& \bar{C}_{H_{\text {outer }}}=C_{0}-\Delta \bar{C}_{H} / 2
\end{aligned}
$$

Over bar indicates the average values. $C_{0}$ is the average of the $\bar{C}_{H_{\text {inner }}}$ and $\bar{C}_{H_{\text {outer }}}$, and is shown in Figure 7.11 by a thin solid black line. $\Delta \bar{C}_{H}$ is the difference between $C_{H}$ of the inner and the outer wings averaged over a half stroke. Equation 7.5 assumes the force coefficient of one wing increases as much as the other one's decreases. The total passive yaw torque exerted on the insect body is then described by Equation 7.6.

$$
\tau_{P P A}=0.5 \rho_{f} S_{w} L_{w}^{2} l C_{0}\left[\left(1+\Delta \hat{C}_{H} / 2\right)(\omega-\Omega)^{2}-\left(1-\Delta \hat{C}_{H} / 2\right)(\omega+\Omega)^{2}\right]
$$

$l$ is the distance of the center of the action of the force along the wing span from the wing base. The hat sign indicates the normalized horizontal force coefficient, $\Delta \hat{C}_{H}=\Delta C_{H} / C_{0}$. This value shows the difference between the force coefficient of the inner and the outer wings normalized by their average value. For instance at $\Omega / \omega=0.2$ the force coefficient of the inner wing is enhanced and that of the outer wing in reduced by about $25 \%$ of the average value, $C_{0}$. Naming the term inside the bracket as $f(\Omega / \omega)$ and assuming $(\Omega / \omega)^{2} \ll 1$, we can write:

$$
f(\Omega / \omega) \approx-4 \omega \Omega+\Delta \hat{C}_{H} \omega^{2}
$$

if $C=0.5 \rho_{f} S_{w} L_{w}^{2} l C_{0}$ and $\Delta \hat{C}_{H}=\delta \omega / \Omega$ we can write: 


$$
\tau_{P P A} \approx-4 C \omega^{2} \frac{\Omega}{\omega}+\delta C \omega^{2} \frac{\Omega}{\omega}
$$

The first and the second terms on the right hand side of the Equation 7.8 are the yaw torque on the body due to FCT and PPA, respectively. Equation 7.8 implies that the magnitude of the torque due to PPA increases linearly with increase in the body velocity (or $\Omega / \omega$ ). The magnitude of the $\tau_{P P A}$ rises with a similar rate as that of the $\tau_{F C T}$, which is strongly desirable for the performance of the maneuver. The relative magnitude of these two terms is $\delta . \delta$ is 2.6 for the wing morphology and kinematics of the damselfly in our experiment which suggests the magnitude of the torque due to the passive wing pitch asymmetry is about $65 \%$ of that of the FCT. Next, we compare the prediction of the yaw velocity half-life by the (only) FCT model, $t_{F C T}^{1 / 2}$, and the complete model which, as well, includes the effect of PPA, $t_{F C T}^{1 / 2}$. We can write:

$$
\begin{aligned}
t_{1 / 2}^{F C T} & =\frac{\ln (2)}{4 C \omega / I_{b}} \\
t_{1 / 2}^{P P A} & =\frac{\ln (2)}{(4-\delta) C \omega / I_{b}}
\end{aligned}
$$

where $I_{b}$ is the body moment of inertia.

$$
\frac{t_{1 / 2}^{P P A}}{t_{1 / 2}^{F C T}}=\frac{4}{4-\delta}
$$

The proposed model predicts the yaw velocity half-life of the damselfly to be about 2.2 wingbeats which is in close agreement with the experimentally measured value (Figure 7.12). Figure 7.12 shows the measured yaw velocity in phase-II together with the prediction of new and FCT model for damselfly $\# 1$. Not only the yaw velocity half-life but also the slope of the variation of the yaw velocity with time is accurately predicted by the new model. The measured yaw velocity half-life for all three damselflies are compared with the predictions of FCT and PPA in Table 7.3. The excellent agreement of the prediction of PPA with experimentally measured data indicates that the morphology and the kinematics of the damselfly wings allow the insect to maintain its body rotational velocity in spite of the strong damping effect due to FCT. 


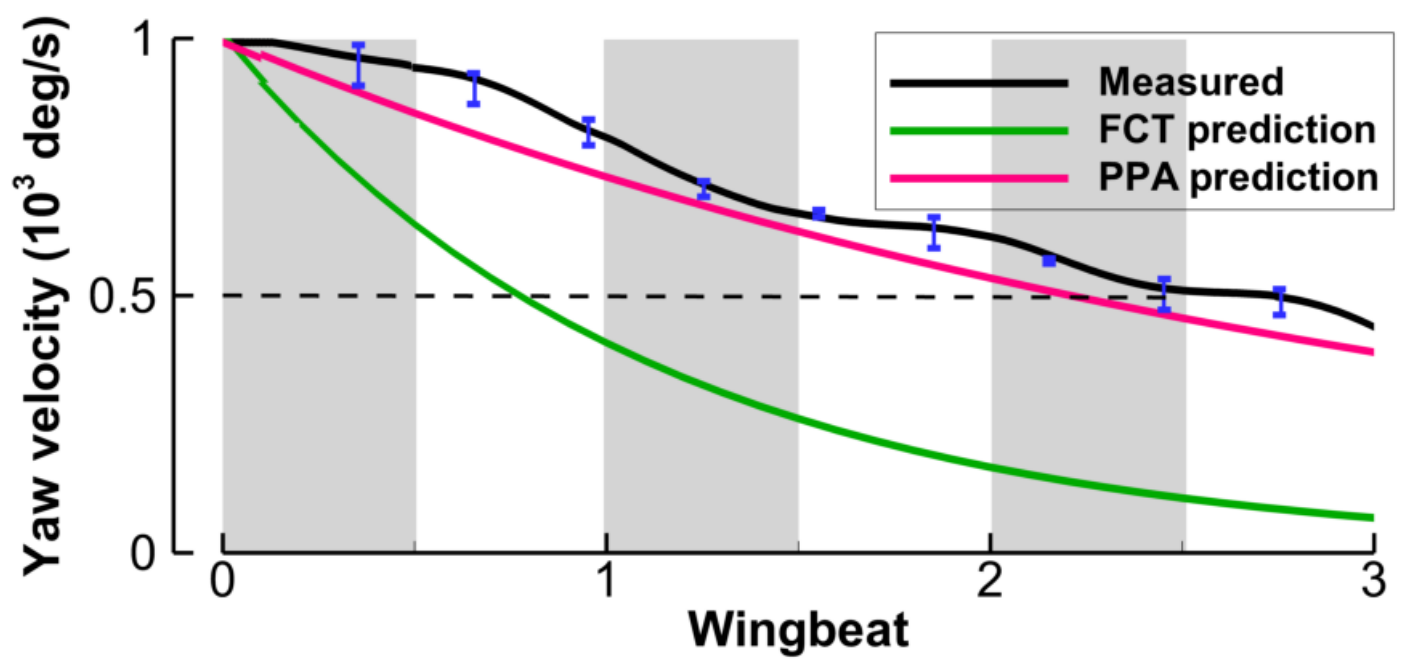

Figure 7.12: Measured and predicted yaw velocity. The normalized body yaw velocity predicted by FCT (dark green) and the new model including the effect of PPA (deep pink) together with the measured value (black) versus the wingbeats for phase-II of the damselfly maneuver.

\begin{tabular}{l|ccc}
\hline & \multicolumn{3}{|c|}{ Yaw velocity half-life } \\
\hline & Measured & Predicted by FCT & Predicted by PPA \\
\hline Damselfly \#1 & 2.5 & 0.7 & 2.2 \\
Damselfly \#2 & 1.5 & 0.6 & 1.6 \\
Damselfly \#3 & 1.0 & 0.4 & 1.1 \\
\hline
\end{tabular}

Table 7.3: Measured and predicted yaw velocity half-life for three damselfly species

\subsection{Broader application of the wing-body coupling in insect flight}

In the previous sections we showed how the motion of the body can cause passive asymmetry in the bilateral wing pitch angles of damselflies during aerial yaw turns. Furthermore, we showed that the passive variation in the wing pitch angles changes the aerodynamic state of the wing and therefore generates torque about the body center of mass. These results, for the first time, show that the dynamics of the wing and the body are coupled in insect flight, suggesting revision and modification of the current models of the insect flight dynamics. Note that although we showed experimental evidence of the wing-body coupling in a single type of motion and only for a specific insect, this does not imply that our conclusions are limited to only this type of motion neither to this specific 
insect. Although we have no means of providing experimentally evidence for all insects and all possible motions, our theoretical modeling that was presented in the previous as well as the current chapter, suggests that wing-body coupling maybe wide spread in insect flight and its strength may increase as the time scales of the wing and the body motions approach.

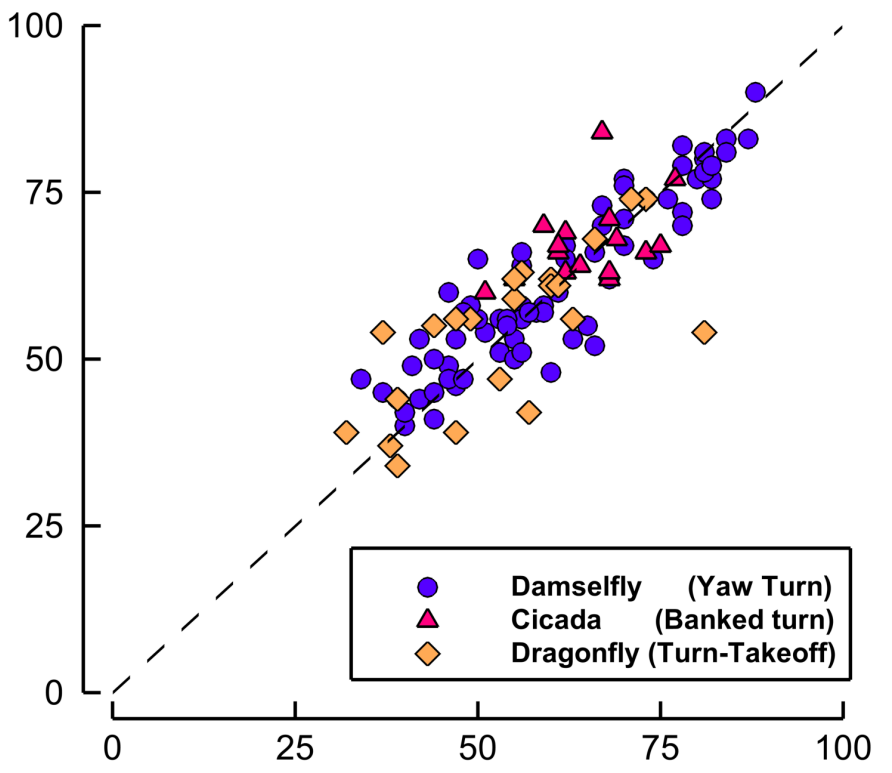

Figure 7.13: Predicted versus measured wing pitch angle in insect maneuvers. The wingbody coupling model is used to predict the wing pitch angle of the damselfly in yaw turn, dragonfly in turning takeoff and cicada in banked turn. The results are shown versus the experimentally measured values.

To examine whether wing-body coupling occurs in the aerial maneuvers of other insects, we used our model to predict the wing pitch angles of a dragonfly in a turning takeoff and a cicada in a banked turn. Similar to the yaw turns of the damselflies, the acceleration and deceleration phases of the maneuver were determined by the timing at which the maximum angular velocity of the body was reached. The flapping motion of the wing in the stroke plane was prescribed as well as the half-stroke-averaged yaw and roll velocities of the body. The inclination of the stroke plane relative to the body was considered in the calculations as well. We then simulated the motion of the motion of the wing and compared the half-stroke-averaged wing pitch angles with the experimentally measured values. Figure 7.13 shows the predicted versus measured wing pitch angles for all three species of insects; damselfly, dragonfly and cicada. Our model can accurately predict the wing pitch 
angles of different insects regardless of the differences in their morphology as well as the wing and the body kinematics, indicating that the wing-body coupling occurs across a wide range of motions in insect flight.

\subsection{Energy saving due to PPA}

Wing-body coupling allows kinetic energy of the body to be stored in the elastic structure of the wing and its hinge. This potential energy is returned to the system by actuating the wing which generates yaw torque about the body axis. Our results indicate that wing-body coupling improves energy economy of the maneuvering flight by reducing damping (energy loss) and by eliminating the need to actively actuate the wing kinematics. Although it is difficult to estimate energy input required for active control, the reduction in the energy loss due to energy recycling can be calculated by multiplying each equation in the set of equations of motion by its characteristic velocity and by subsequent integration. In the case of pure yaw motion energy can be calculated by Equation 7.12.

$$
\int_{0}^{t} I_{z z} \dot{R} R d t=\int_{0}^{t} N R d t
$$

where $N$ is the aerodynamic yaw torque and $R$ is the yaw velocity. Left hand side term integrates into kinetic energy expression. The term in the integral on the right hand side is the rate of energy gain/loss of the system. We showed that aerodynamics yaw torque in linearly proportional to the yaw velocity for both cases with and without PPA. If $N_{F C T}=-4 C \omega R$ and $N_{P P A}=-(4-\delta) C \omega R$, the kinetic energy of the system at time $t, \epsilon(t)$, can be calculated as follows:

$$
\begin{aligned}
& \epsilon(t)_{F C T}=0.5 I_{z z} R_{\text {max }}^{2}-4 C \omega \int_{0}^{t} R(t)^{2} d t \\
& \epsilon(t)_{P P A}=0.5 I_{z z} R_{\text {max }}{ }^{2}-(4-\delta) C \omega \int_{0}^{t} R(t)^{2} d t
\end{aligned}
$$

Normalizing energy by its maximum value, $0.5 I_{z z} R_{\max }{ }^{2}$, and knowing that the yaw velocity drops exponentially, $R_{F C T}=e^{\frac{-4 C \omega t}{I_{z z}}}$ and $R_{P P A}=e^{\frac{-(4-\delta) C \omega t}{I_{z z}}}$, the energy of the system at time $t$ is expressed by Equation 7.13. 


$$
\begin{aligned}
& \epsilon(t)_{F C T}=e^{2\left(\frac{-4 C \omega t}{I_{z z}}\right)} \\
& \epsilon(t)_{P P A}=e^{2\left(\frac{-(4-\delta) C \omega t}{I_{z z}}\right)}
\end{aligned}
$$

Time history of kinetic energy of the system as predicted by FCT (in red) and PPA (in green) is shown in Figure 7.14. The gray shaded area is the total energy if there was no damping. The total kinetic energy utilized by the system to change the flight direction can be calculated from the area under each one of the curves. The difference between the area under the curve and the gray shaded area is the energy loss due to damping. It is evident from the graphics that wing-body coupling reduced energy loss. Integrating Equation 7.13 in time, from 0 to $t$ (with 0 being the beginning of deceleration phases where the maximum yaw velocity is reached) allows calculation of the total kinetic energy of the system (Equation 7.14).

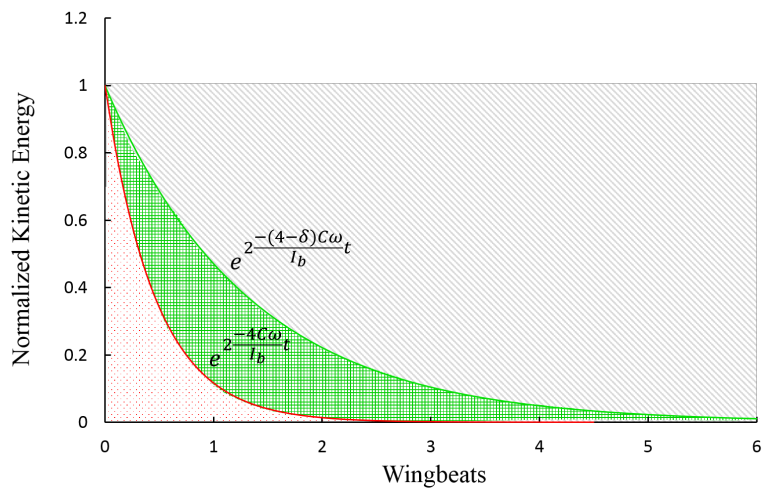

Figure 7.14: Kinetic energy decay with and without wing-body coupling in yaw turns.

$$
\begin{aligned}
& E_{F C T}=\frac{-2 I_{z z}}{4 C \omega t}\left(1-e^{2\left(\frac{-4 C \omega t}{I_{z z}}\right)}\right) \\
& E_{P P A}=\frac{-2 I_{z z}}{(4-\delta) C \omega t}\left(1-e^{2\left(\frac{-(4-\delta) C \omega t}{I_{z z}}\right)}\right)
\end{aligned}
$$


Selecting the upper limit of the integral large enough where the body velocity has dropped to near zero values, the ratio of the kinetic energy of the system with PPA is $\frac{4}{(4-\delta)}$ times that of the system without PPA. For instance for damselfly \#1, PPA resulted in at least $185 \%$ improvement in the energy consumption of the system. This estimation only accounts for the energy recycling due to the wing-body coupling. In addition, passive actuation of the wing kinematics eliminates the need for active control and therefore improves energy consumption even more.

Our aforementioned analysis shows the importance of PPA in improving both performance and energy economy of insect flight during a yaw turn. Although yaw turns were frequently observed to be employed by different insect species for changing the flight direction, aerial maneuvers of the insects are usually more complex involving simultaneous rotations about all three axes of the body. Due to nonlinearity of the rigid body equations of motion, it is not clear how PPA affects energy economy of the system during complex aerial maneuvers. Similar to the pure yaw motion, expression of the energy contribution of each term in equations of motion can be obtained by multiplying each equation with its characteristic velocity and integrating in time (Equation 7.15).

$$
\begin{aligned}
& \int_{0}^{t}\left[I_{x x} \dot{P} P+Q r\left(I_{z z}-I_{y y}\right) P-I_{x z}(\dot{r}+P Q) P\right] d t=\int_{0}^{t} L P d t \\
& \int_{0}^{t}\left[I_{y y} \dot{Q} Q+\operatorname{Pr}\left(I_{x x}-I_{z z}\right) Q+I_{x z}\left(P^{2}-r^{2}\right) Q\right] d t=\int_{0}^{t} M Q d t \\
& \int_{0}^{t}\left[I_{z z} \dot{R} R+P Q\left(I_{y y}-I_{x x}\right) R+I_{x z}(Q R-\dot{P}) R\right] d t=\int_{0}^{t} N R d t
\end{aligned}
$$

where $P$ and $Q$ are roll and pitch velocities, respectively. $L$ and $M$ are the torque components about the roll and pitch body axes. Summing up the three equations in 7.15, one can recover the statement of energy balance for the system (Equation 7.16), with the left hand side of the equation being the kinetic energy of the system (note that most nonlinear terms cancel out).

$$
\left[0.5 I_{x x} P^{2}+0.5 I_{y y} Q^{2}+0.5 I_{z z} R^{2}-I_{x z} P R\right]_{0}^{t}=\int_{0}^{t} L P d t+\int_{0}^{t} M Q d t+\int_{0}^{t} N R d t
$$

where terms in the integral of the right hand side are the rates of energy gain/loss of the system. The left hand side term expresses the instantaneous level of the system kinetic energy. Equation 7.16 implies that despite nonlinearity of the equations of motion, rate of total energy loss is the sum 
of the rates of energy loss about each body axes, indicating that the energy saving due to PPA can be linearly summed up to calculate total energy saving of the system in a more complex motion.

In each direction the aerodynamic torque is linearly proportional to the corresponding rotational velocity. By integrating Equation 7.16 we obtain a similar expression for the energy state of the system as was derived for pure yaw motion in Equation 7.13.

$$
\begin{aligned}
& \epsilon(t)_{F C T}=0.5 I_{x x} P_{\max }^{2} e^{2\left(\frac{-4 C_{\text {roll } \omega t}}{I_{b}}\right)}+0.5 I_{y y} Q_{\max }{ }^{2} e^{2\left(\frac{-4 C_{p i t c h} \omega t}{I_{b}}\right)}+0.5 I_{z z} R_{\text {max }} e^{2\left(\frac{-4 C_{y a w \omega t}}{I_{b}}\right)} \\
& \epsilon(t)_{P P A}=0.5 I_{x x} P_{\max } e^{2\left(\frac{-\left(4-\delta_{\text {roll }}\right) C_{\text {roll }} \omega t}{I_{b}}\right)}+0.5 I_{y y} Q_{\max } e^{2\left(\frac{-\left(4-\delta_{\text {pitch }}\right) C_{\text {pitch }} \omega t}{I_{b}}\right)}+0.5 I_{z z} R_{\text {max }} e^{2\left(\frac{-\left(4-\delta_{\text {yaw }}\right) C_{\text {yaw }} \omega t}{I_{b}}\right)}
\end{aligned}
$$

Total energy of the system is sum of three exponentially decaying energy equations with different scales. Our analysis shows that an insect can benefit from the energy saving properties of PPA regardless of the complexity of the motion.

\subsection{Conclusions}

The current work reveals the critical importance of considering the effect of the body motion on modulating the wing motion. We particularly investigated the connection between the wing and the body dynamics of 3 different species of damselflies in yaw turn. Our results show that the coupling between the wing and the body dynamics gives rise to passive alternation in the bilateral wing pitch angles when the insect body is engaged in a fast yaw turn. Specially, we showed that a damselfly dominantly uses this passive mechanism for maintaining the bilateral wing pitch asymmetry that generates the aerodynamic yaw torque for keeping the body in rotation. We further showed that the conclusions on the coupling in the dynamics of the wing and the body hold true for other insects as well as other types of motion.

Taken together, these findings emphasize the important role that the aerodynamics of flapping flight plays in the wing kinematics control. Previously, many studies investigated the effect of the wing motion on generating the unsteady aerodynamic forces in insect flight [Sane and Dickinson, 2002, Ellington, 1994]. However, there is much to be learned about the reverse relationship. Wings of the insects are in constant interaction with the surrounding air, even though they are powered from within the thorax by the flight muscles. Here we showed that the combination of the wing hinge 
flexibility and the aerodynamics of flapping flight allows modulations of the wing motion without additional assistance from the flight muscles in the thorax. Yet, more studies are necessary to unravel the mutual relationship between these two aspects of flapping flight. 


\section{Chapter 8}

\section{Conclusions}

\subsection{Summary of contributions}

An integrative approach to the study of insect flight focuses on the interactions and connections of different components of the flight such as the wings, body, sensory network, neuro-muscular system and etc. Each one of these systems as well their interactions are complex and the work towards understanding them is far from finished yet. In this thesis, we analyzed the connection of the two most important components of flight, the wing and body, from dynamics point of view.

A summary of the conclusions of each chapter is provided below;

In chapter 3, turn takeoff flight of several dragonflies was recorded, using a high speed photogrammetry system. We investigated strength of the dynamic coupling by dividing pitch, roll and yaw angular accelerations into two contributions; one from aerodynamic torque and one from dynamic coupling effect. The latter term is referred to as Dynamic Coupling Acceleration (DCA). The DCA term can be measured directly from instantaneous rotational velocities of the insect. By means of a simple conceptual model, and further by our experimental measurements, we showed how DCA can change the orientation of the body during the flight. In the measured flight data, a strong correlation was found between the pitch velocity at the end of each wingbeat and the time integral of the corresponding DCA term. Our results suggest that pitching up and down motion, which was a characteristic of this maneuvering flight, is partially controlled via dynamic coupling rather than relying only on fine adjustments in the aerodynamic torque. This implies application of a simpler control strategy for execution of a three dimensional turn during which the existing 
kinetic energy of the system can be employed to manipulate the motion. We argued that employing this mechanism allows executions of a variety of maneuvers without fine adjustment over the aerodynamic torque.

In chapter 4, the measured wing and body kinematics of cicada (Tibicen linnei) in free flight was presented. We covered a vast range of flights including flight periods of both little and substantial body reorientations. It was found that cicadas employ a common force vectoring technique to execute all these flights. We showed that the direction of the half-stroke averaged aerodynamic force relative to the body is independent of the body orientation, varying in a range of merely 20 deg. Despite directional limitation of the aerodynamic force, pitch and roll torque were generated by altering wing angle of attack and its mean position relative to the center of mass. This resulted in body rotations which redirect the wing force in the global frame and thus change the flight trajectory.

Chapter 5 presented measurements of the wing and body kinematics of three different species of damselflies in yaw turn. Although there have been numerous studies on the aerial maneuvers of a variety of insects, there is a lack of sufficient information on both the measurements and analysis of free flight of damselflies. Therefore this study helps filling this gap by providing these measurements. The yaw turn was characterized by a short acceleration phase which is immediately followed by an elongated deceleration phase. Most of the heading change takes place during the latter stage of the flight. Our observations showed that yaw turns are executed via drastic rather than subtle changes in the kinematics of all four wings. The motion of the inner and the outer wings were found to be strongly linked through their orientation as well as the velocities with inner wings moving faster than the outer wings. The wing angle of attack exerted the strongest influence on the yaw torque, followed by the flapping and deviation velocities of the wings. Moreover, no evidence of active generation of counter torque was found in the flight data implying that deceleration and stopping of the maneuver is dominated by passive damping.

Chapter 6 quantitatively analyzed behavior of the insect wing pitching by combining models of the wing biomechanics and the unsteady aerodynamics of flapping flight. We showed that the half-stroke-averaged angle of attack as well as the force coefficient vary by the wing aspect ratio and the ratio of the aerodynamic to elastic force $(C h)$. Across a specific range of these dimensionless numbers, high lift production can be achieved. The results of this quantitative analysis can be used 
to inform design of flapping wings. Moreover, our model predicts that the body motion induces passive changes in the wing kinematics by altering effective $C h$ of the wing. Consequently, the aerodynamic force coefficient changes which leads to adjusting the body motion. This chain of actions and interactions results in coupling of the dynamics of the wing and the body. Finally, our analysis suggest that the current model of the insect flight dynamics is inaccurate and should be modified to incorporate the wing-body interactions.

Chapter 7 investigated the connection between the wing and the body motion in insect free flight via analyzing fast yaw turns of three different species of damselflies. The measurements indicated that, during a fast yaw turn, the angular velocity of the damselfly body stays within 15\%-30\% of that of the wing throughout most of the maneuver duration, indicating the proximity of the time scales of the wing and body motions. In this combined experimental and theoretical study, we showed that the dynamics of the wings and the body of a damselfly are coupled together. When the insect is engaged in a fast yaw turn, the rotation of the body causes the aerodynamic pitching torque, about the wing pitching axis, to increase on one wing and decrease on the other. This generates asymmetric pitch angles on the bilateral wings. As a result a damselfly is able to maintain the yaw torque that keeps the body in rotation. Furthermore, our model was able to accurately predict the wing pitch angles of a dragonfly in turning takeoff and cicada in banked turn, indicating the broader application of the proposed model across different flight modes and insect species.

Taken together, the contribution of this thesis is threefold:

(1) Investigation of the free flight mechanics of insects. Direct observation and measurement of the free flight is one of the best approach to understanding the flight mechanics. Recent advents in the high speed videography and accurate tracking techniques made these investigations possible. In this thesis, we studied free flight of several species of insects; dragonflies, damselflies and cicadas. All these insects have unique flying features. Dragonflies and damselflies are among the most maneuverable insects with two functionally independent pairs of wings. Cicada is a simpler flier with large wing loading and yet a wide range of flight capabilities. Some of the data gathered on the free flight of these insects, as well as the flight mechanisms revealed in our study are among the first of their kind to be reported.

(2) Provide guidelines for Micro Air Vehicle (MAV) design. The results of this thesis not only provide a better understanding of the insect flight dynamics but many of the lessons learned can 
be applied to the design of agile MAVs. For instance, in chapter 4, our measurements and analysis of the free flight of cicada indicated that a wide range of flight modes can be achieved by a simple control strategy that involves minor modulations in the wing stroke plane. A similar technique can be implemented in design of MAV, providing the desired flight capacity and simplifying the mechanics of the wing. In addition, the results of our analysis in chapters 6 and 7 promote application of flexible wing structure in design of agile flapping wing MAV's. We showed that the wing kinematics as well as the flight force can be effectively controlled if the structural properties of the wing hinge is appropriately selected in harmony with the wing morphology and kinematics.

(3) Proposing a more accurate model of the insect flight dynamics. Our work towards understanding the flapping wing dynamics and basics of wing actuation has led to improving our understanding of the insect flight as a system. The results of our analysis indicated that the body motion alters the balance of forces on the wing causing the wing motion to change even in the absence of active control. This phenomenon, that was predicted theoretically and verified experimentally in this thesis, cannot be explained by the current model of the insect flight dynamics. To incorporate this effect, we proposed a new model that integrates the wing into the insect flight dynamics. The validity of the proposed model and the accuracy of its predictions were verified through direct measurement of the wing and body kinematics of several insect species in free flight.

\subsection{Suggestions for future work}

This thesis provides an incremental advancement of our understanding of the insect flight dynamics by investigating the integrative dynamics of the wing and the body. However, it is by no means an exhaustive research and there is room left to improve and advance this work.

Due to the complexity of the problem, several simplifications were made in modeling the wing geometry and its biomechanics as well as the aerodynamic force and torque predictions. For instance, a quasi-steady approximation of the aerodynamic force and torque was applied to simplify the numerical simulations of the wing-air interactions. However, the range of accuracy of this model is limited. Although the major conclusions of this work hold, regardless of these potential inaccuracies, more accurate force calculation methods such as Computational Fluid Dynamics (CFD) can be applied to improve the accuracy of the predictions. In addition, our experimental measurements 
were limited to a few species of insects. The experimental data pool can be potentially expanded to include other insect species and a wider variety of aerial maneuvers. One of the main challenges faced, regarding the expansion of the experimental database, was the tediousness of the manual tracking technique that was employed to extract the flight data from the high speed videos. Currently, on average, it takes about 30 hours to reconstruct $100 \mathrm{~ms}$ of flight videos. In low flapping frequency flight, that is only 3-4 wingbeats compared to 10-12 wingbeats for high flapping frequency insects such as fruit flies. Investing on developing automatic or semi-automatic tracking algorithms can help eliminating this barrier.

Here, listed are three possible branches that the current work could be extended to:

(1) Revising assumptions of constant wing kinematics in the study of insect flight dynamics. Active control of the wing motion requires neural and mechanical feedback from the body motion as well as feedforward motor neurons to transport the control signal to the flight muscle. Therefore, any adjustment in the wing kinematics in response to an internally or externally induced change in the flight condition will be delayed inevitably. This also implies that the openloop dynamics of the flight is driven by the pre-disturbance kinematics of the wings which remains constant until active control commands arrive to the driving muscles. Our analysis, in this thesis, showed that there is an alternative passive pathway by which changes in the flight condition can affect the wing kinematics. These immediate changes cause alternations in the flight force and consequently transform the response of the insect. Therefore, it is important to consider wing-body coupling in the investigations of both open-loop and close-loop dynamics of insect free flight.

(2) Investigating the effect of wing surface flexibility on wing-body coupling. The biomechanics of the insect wing is complex. To simplify the analysis, in this work, we assumed that the wing surface is rigid and all the torsional flexibility of the wing is concentrated at its hinge. However, the flexibility of the wing surface can potentially play a similar role as that of the wing hinge, meaning that the body motion can induce passive twisting of the wing surface. Investigation of this problem requires more complex structure models that can mimic the flexibility of the veins along the wing length. A possible model would involve a rigid wing surface that is connected to a variable cross section beam at its leading edge. The aerodynamic load on the wing causes the beam, and consequently the wing, to twist. Moreover, more elaborate numerical modeling of the flow structure interactions need to be implemented in this problem. 
(3) Extension of the conclusions to swimming animals. Flying and swimming animals share many similarities in the kinematics and morphology of the wings/fins. Similar to the wings, the propulsive surfaces of swimming animals experience passive deformations during locomotion. For instance, the propulsive performance of pectoral fins is function of the surface stiffness and its deformations [Moored et al., 2011]. It is therefore possible that an analogous coupling between the fin and body dynamics occur. A similar investigation of this phenomenon, in swimming, can be conducted employing the techniques and methods that were developed in this thesis. However, a more accurate model of the fin structure may be needed. 


\section{Bibliography}

[Kem, ] Investigating Batoid-Inspired Propulsion: The Development, Testing, and Performance Analysis of a Tensegrity-Based Robotic Fin for Underwater Locomotion. PhD thesis, University of Virginia. 10

[Alexander, 1986] Alexander, D. E. (1986). Wind tunnel studies of turns by flying dragonflies. Journal of Experimental Biology, 122(1):81-98. 25, 47, 64, 67, 69, 82, 115

[Altshuler et al., 2005] Altshuler, D. L., Dickson, W. B., Vance, J. T., Roberts, S. P., and Dickinson, M. H. (2005). Short-amplitude high-frequency wing strokes determine the aerodynamics of honeybee flight. Proceedings of the National Academy of Sciences of the United States of America, 102(50):18213-18218. 65

[Andersen et al., 2005] Andersen, A., Pesavento, U., and Wang, Z. J. (2005). Unsteady aerodynamics of fluttering and tumbling plates. Journal of Fluid Mechanics, 541:65-90. 50, 98, 99

[Balint and Dickinson, 2001] Balint, C. N. and Dickinson, M. H. (2001). The correlation between wing kinematics and steering muscle activity in the blowfly calliphora vicina. Journal of experimental biology, 204(24):4213-4226. 20, 67

[Balint and Dickinson, 2004] Balint, C. N. and Dickinson, M. H. (2004). Neuromuscular control of aerodynamic forces and moments in the blowfly, calliphora vicina. Journal of Experimental Biology, 207(22):3813-3838. 67

[Bastian, 1972] Bastian, J. (1972). Neuro-muscular mechanisms controlling a flight maneuver in the honeybee. Journal of comparative physiology, 77(2):126-140. 67

[Bender and Dickinson, 2006] Bender, J. A. and Dickinson, M. H. (2006). Visual stimulation of saccades in magnetically tethered drosophila. The Journal of experimental biology, 209(16):3170-3182. 67

[Berger and Kutsch, 2003] Berger, S. and Kutsch, W. (2003). Turning manoeuvres in free-flying locusts: High-speed video-monitoring. Journal of Experimental Zoology Part A: Comparative Experimental Biology, 299(2):127-138. 25

[Bergou et al., 2010] Bergou, A. J., Ristroph, L., Guckenheimer, J., Cohen, I., and Wang, Z. J. (2010). Fruit flies modulate passive wing pitching to generate in-flight turns. Physical review letters, 104(14):148101. 8, 21, 48, 49, 67, 69, 76, 77, 82, 85, 88, 89, 95, 97, 105, 107, 112, 115, 116,123

[Bergou et al., 2007] Bergou, A. J., Xu, S., and Wang, Z. (2007). Passive wing pitch reversal in insect flight. Journal of Fluid Mechanics, 591:321-337. 7, 11, 21, 94, 98, 99, 115 
[Betts and Wootton, 1988] Betts, C. and Wootton, R. (1988). Wing shape and flight behaviour in butterflies (lepidoptera: Papilionoidea and hesperioidea): a preliminary analysis. Journal of Experimental Biology, 138(1):271-288. 6

[Birch and Dickinson, 2001] Birch, J. M. and Dickinson, M. H. (2001). Spanwise flow and the attachment of the leading-edge vortex on insect wings. Nature, 412(6848):729-733. 12

[Birch and Dickinson, 2003] Birch, J. M. and Dickinson, M. H. (2003). The influence of wingwake interactions on the production of aerodynamic forces in flapping flight. Journal of Experimental Biology, 206(13):2257-2272. 13

[Birch et al., 2004] Birch, J. M., Dickson, W. B., and Dickinson, M. H. (2004). Force production and flow structure of the leading edge vortex on flapping wings at high and low reynolds numbers. Journal of Experimental Biology, 207(7):1063-1072. 12

[Bisplinghoff et al., 2013] Bisplinghoff, R. L., Ashley, H., and Halfman, R. L. (2013). Aeroelasticity. Courier Corporation. 99

[Bode Oke et al., 2015] Bode Oke, A., Zeyghami, S., and Dong, H. (2015). Optimized body deformation in dragonfly maneuvers. AIAA paper. 16, 17

[Camhi, 1970] Camhi, J. M. (1970). Sensory control of abdomen posture in flying locusts. J. exp. Biol, 52(3):533. 17

[Cheng and Deng, 2010] Cheng, B. and Deng, X. (2010). Near-hover dynamics and attitude stabilization of an insect model. In American Control Conference (ACC), 2010, pages 39-44. IEEE. 19

[Cheng and Deng, 2011] Cheng, B. and Deng, X. (2011). Translational and rotational damping of flapping flight and its dynamics and stability at hovering. Robotics, IEEE Transactions on, 27(5):849-864. 2, 90

[Cheng et al., 2011] Cheng, B., Deng, X., and Hedrick, T. L. (2011). The mechanics and control of pitching manoeuvres in a freely flying hawkmoth (manduca sexta). The Journal of experimental biology, 214(24):4092-4106. 19, 42

[Cheng et al., 2010] Cheng, B., Fry, S., Huang, Q., and Deng, X. (2010). Aerodynamic damping during rapid flight maneuvers in the fruit fly drosophila. The Journal of experimental biology, 213(4):602-612. 5

[Cobb, 2010] Cobb, M. (2010). The damselfly enigma: Better bigger or smaller? The Journal of Experimental Biology, 213(17):vi-vi. 67

[Combes and Daniel, 2003] Combes, S. A. and Daniel, T. L. (2003). Flexural stiffness in insect wings i. scaling and the influence of wing venation. Journal of Experimental Biology, 206(17):2979-2987. 14, 95

[Combes and Dudley, 2009] Combes, S. A. and Dudley, R. (2009). Turbulence-driven instabilities limit insect flight performance. Proceedings of the National Academy of Sciences, 106(22):91059108. 30

[Combes et al., 2012] Combes, S. A., Rundle, D., Iwasaki, J., and Crall, J. (2012). Linking biomechanics and ecology through predator-prey interactions: flight performance of dragonflies and their prey. The Journal of experimental biology, 215(6):903-913. 6, 25 
[Daniel and Combes, 2002] Daniel, T. L. and Combes, S. A. (2002). Flexible wings and fins: bending by inertial or fluid-dynamic forces? Integrative and Comparative Biology, 42(5):10441049. 94,123

[David, 1978] David, C. T. (1978). The relationship between body angle and flight speed in freeflying drosophila. Physiological Entomology, 3(3):191-195. 65

[de Croon et al., 2012] de Croon, G. C., Groen, M., De Wagter, C., Remes, B., Ruijsink, R., and van Oudheusden, B. W. (2012). Design, aerodynamics and autonomy of the delfly. Bioinspiration \& biomimetics, 7(2):025003. 22

[Deng et al., 2006] Deng, X., Schenato, L., Wu, W. C., and Sastry, S. S. (2006). Flapping flight for biomimetic robotic insects: Part i-system modeling. Robotics, IEEE Transactions on, 22(4):776-788. 105, 121

[Dickinson, 2003] Dickinson, M. (2003). Animal locomotion: how to walk on water. Nature, 424(6949):621-622. 115

[Dickinson, 2005] Dickinson, M. H. (2005). The initiation and control of rapid flight maneuvers in fruit flies. Integrative and comparative biology, 45(2):274-281. 77, 82

[Dickinson et al., 2000] Dickinson, M. H., Farley, C. T., Full, R. J., Koehl, M., Kram, R., and Lehman, S. (2000). How animals move: an integrative view. Science, 288(5463):100-106. 90

[Dickinson and Gotz, 1993] Dickinson, M. H. and Gotz, K. G. (1993). Unsteady aerodynamic performance of model wings at low reynolds numbers. The Journal of Experimental Biology, 174(1):45-64. 19, 104

[Dickinson et al., 1998] Dickinson, M. H., Lehmann, F.-O., and Chan, W. P. (1998). The control of mechanical power in insect flight. American zoologist, 38(4):718-728. 20

[Dickinson et al., 1993] Dickinson, M. H., Lehmann, F.-O., and Gotz, K. (1993). The active control of wing rotation by drosophila. The Journal of experimental biology, 182(1):173-189. 13, $69,95,115,117$

[Dickinson et al., 1999] Dickinson, M. H., Lehmann, F.-O., and Sane, S. P. (1999). Wing rotation and the aerodynamic basis of insect flight. Science, 284(5422):1954-1960. 11, 13, 15, 67

[Dickson et al., 2006] Dickson, W. B., Straw, A. D., Poelma, C., and Dickinson, M. H. (2006). An integrative model of insect flight control. In Proceedings of the 44th AIAA Aerospace Sciences Meeting and Exhibit, pages 31-38. 97

[Dillon and Dudley, 2004] Dillon, M. E. and Dudley, R. (2004). Allometry of maximum vertical force production during hovering flight of neotropical orchid bees (apidae: Euglossini). Journal of Experimental Biology, 207(3):417-425. 64, 65

[Dong and Liang, 2010] Dong, H. and Liang, Z. (2010). Effects of ipsilateral wing-wing interactions on aerodynamic performance of flapping wings. In Proceedings of 48 th AIAA aerospace sciences meeting including the new horizons forum and aerospace exposition, pages 2010-71. 13

[Dudley, 2002] Dudley, R. (2002). The biomechanics of insect flight: form, function, evolution. Princeton University Press. 5, 6, 7, 8, 11, 13, 16, 21, 26, 67, 95, 117 
[Dudley and Ellington, 1990] Dudley, R. and Ellington, C. (1990). Mechanics of forward flight in bumblebees: I. kinematics and morphology. Journal of Experimental Biology, 148(1):19-52. 8

[Dyhr et al., 2013] Dyhr, J. P., Morgansen, K. A., Daniel, T. L., and Cowan, N. J. (2013). Flexible strategies for flight control: an active role for the abdomen. The Journal of experimental biology, 216(9):1523-1536. 26

[Ellington, 1984a] Ellington, C. (1984a). The aerodynamics of hovering insect flight. ii. morphological parameters. Philosophical Transactions of the Royal Society of London. B, Biological Sciences, 305(1122):17-40. 7

[Ellington, 1984b] Ellington, C. (1984b). The aerodynamics of hovering insect flight. iv. aeorodynamic mechanisms. Philosophical Transactions of the Royal Society B: Biological Sciences, 305(1122):79-113. 6, 10, 14, 64, 65, 98, 112

[Ellington, 1991] Ellington, C. (1991). Limitations on animal flight performance. Journal of Experimental Biology, 160(1):71-91. 47, 64

[Ellington, 1994] Ellington, C. (1994). Unsteady aerodynamics of insect flight. In Symposia of the Society for Experimental Biology, volume 49, pages 109-129. 139

[Ellington, 1999] Ellington, C. P. (1999). The novel aerodynamics of insect flight: applications to micro-air vehicles. Journal of Experimental Biology, 202(23):3439-3448. 8, 11, 65

[Ellington et al., 1996] Ellington, C. P., Van Den Berg, C., Willmott, A. P., and Thomas, A. L. (1996). Leading-edge vortices in insect flight. 11

[Ennos, 1988] Ennos, A. R. (1988). The importance of torsion in the design of insect wings. Journal of Experimental Biology, 140(1):137-160. 7, 21, 94, 105, 115, 123

[Fry et al., 2003] Fry, S. N., Sayaman, R., and Dickinson, M. H. (2003). The aerodynamics of freeflight maneuvers in drosophila. Science, 300(5618):495-498. 6, 8, 25, 47, 49, 64, 67, 69, 76, 82, $88,95,112,115,127$

[Fry et al., 2005] Fry, S. N., Sayaman, R., and Dickinson, M. H. (2005). The aerodynamics of hovering flight in drosophila. Journal of Experimental Biology, 208(12):2303-2318. 47, 48, 49, 64

[Fuller et al., 2014] Fuller, S. B., Straw, A. D., Peek, M. Y., Murray, R. M., and Dickinson, M. H. (2014). Flying drosophila stabilize their vision-based velocity controller by sensing wind with their antennae. Proceedings of the National Academy of Sciences, 111(13):E1182-E1191. 116

[Gaston, 1996] Gaston, K. J. (1996). Spatial covariance in the species richness of higher taxa. Aspects of the genesis and maintenance of biological diversity, pages 221-242. 5

[GOTZ, 1987] GOTZ, K. G. (1987). Course-control, metabolism and wing interference during ultralong tethered flight in drosophila melanogaster. Journal of experimental biology, 128(1):3546. 19

[Gotz et al., 1979] Gotz, K. G., Hengstenberg, B., and Biesinger, R. (1979). Optomotor control of wing beat and body posture in drosophila. Biological Cybernetics, 35(2):101-112. 17, 26

[Gotz and Wehrhahn, 1984] Gotz, K. G. and Wehrhahn, C. (1984). Optomotor control of the force of flight in drosophila and musca. Biological cybernetics, 51(2):129-134. 64 
[Hedrick and Biewener, 2007] Hedrick, T. and Biewener, A. (2007). Low speed maneuvering flight of the rose-breasted cockatoo (eolophus roseicapillus). i. kinematic and neuromuscular control of turning. Journal of Experimental Biology, 210(11):1897-1911. 25, 48

[Hedrick and Daniel, 2006] Hedrick, T. and Daniel, T. (2006). Flight control in the hawkmoth manduca sexta: the inverse problem of hovering. The journal of experimental Biology, 209(16):3114-3130. 30, 67

[Hedrick et al., 2009] Hedrick, T. L., Cheng, B., and Deng, X. (2009). Wingbeat time and the scaling of passive rotational damping in flapping flight. Science, 324(5924):252-255. 33, 37, 47, $48,67,74,88,89,107,116,121,127,131$

[Heide and Gotz, 1996] Heide, G. and Gotz, K. G. (1996). Optomotor control of course and altitude in drosophila melanogaster is correlated with distinct activities of at least three pairs of flight steering muscles. The Journal of experimental biology, 199(8):1711-1726. 67

[Hinterwirth and Daniel, 2010] Hinterwirth, A. J. and Daniel, T. L. (2010). Antennae in the hawkmoth manduca sexta (lepidoptera, sphingidae) mediate abdominal flexion in response to mechanical stimuli. Journal of Comparative Physiology A, 196(12):947-956. 17

[Huxley, 1974] Huxley, A. (1974). Muscular contraction. The Journal of physiology, 243(1):1-43. 20

[Iriarte-Diaz and Swartz, 2008] Iriarte-Diaz, J. and Swartz, S. M. (2008). Kinematics of slow turn maneuvering in the fruit bat cynopterus brachyotis. Journal of Experimental Biology, 211(21):3478-3489. 25, 48

[Ishihara et al., 2009] Ishihara, D., Yamashita, Y., Horie, T., Yoshida, S., and Niho, T. (2009). Passive maintenance of high angle of attack and its lift generation during flapping translation in crane fly wing. Journal of Experimental Biology, 212(23):3882-3891. 11, 21, 94, 98, 100, 105, $115,123,124$

[Jones, 1941] Jones, W. P. (1941). The virtual inertias of a tapered wing in still air. HM Stationery Office. 11

[Kammer, 1971] Kammer, A. E. (1971). The motor output during turning flight in a hawkmoth, manduca sexta. Journal of Insect Physiology, 17(6):1073-1086. 21

[Karban, 1981] Karban, R. (1981). Flight and dispersal of periodical cicadas. Oecologia, 49(3):385-390. 47

[Keennon et al., 2012] Keennon, M., Klingebiel, K., Won, H., and Andriukov, A. (2012). Development of the nano hummingbird: A tailless flapping wing micro air vehicle. In AIAA Aerospace Sciences Meeting, pages 1-24. 22

[Koehler et al., 2012] Koehler, C., Liang, Z., Gaston, Z., Wan, H., and Dong, H. (2012). 3d reconstruction and analysis of wing deformation in free-flying dragonflies. The Journal of experimental biology, 215(17):3018-3027. 6, 25, 27, 28, 50, 95, 116

[Lehmann, 2009] Lehmann, F.-O. (2009). Wing-wake interaction reduces power consumption in insect tandem wings. Experiments in fluids, 46(5):765-775. 13 
[Lehmann and Dickinson, 1997] Lehmann, F.-O. and Dickinson, M. H. (1997). The changes in power requirements and muscle efficiency during elevated force production in the fruit fly drosophila melanogaster. The Journal of experimental biology, 200(7):1133-1143. 6

[Lehmann and Dickinson, 1998] Lehmann, F.-O. and Dickinson, M. H. (1998). The control of wing kinematics and flight forces in fruit flies (drosophila spp.). The Journal of experimental biology, 201(3):385-401. 6, 19, 67, 69, 115

[Lehmann and Dickinson, 2001] Lehmann, F.-O. and Dickinson, M. H. (2001). The production of elevated flight force compromises manoeuvrability in the fruit fly drosophila melanogaster. Journal of experimental biology, 204(4):627-635. 67

[Lentink and Dickinson, 2009] Lentink, D. and Dickinson, M. H. (2009). Rotational accelerations stabilize leading edge vortices on revolving fly wings. Journal of Experimental Biology, 212(16):2705-2719. 12

[Ma et al., 2013] Ma, K. Y., Chirarattananon, P., Fuller, S. B., and Wood, R. J. (2013). Controlled flight of a biologically inspired, insect-scale robot. Science, 340(6132):603-607. 22

[Marden, 1987] Marden, J. H. (1987). Maximum lift production during takeoff in flying animals. Journal of Experimental Biology, 130(1):235-258. 11

[McLachlan et al., 2003] McLachlan, A., Ladle, R., and Crompton, B. (2003). Predator-prey interactions on the wing: aerobatics and body size among dance flies and midges. Animal behaviour, 66(5):911-915. 6, 25

[Moored et al., 2011] Moored, K., Kemp, T., Houle, N., and Bart-Smith, H. (2011). Analytical predictions, optimization, and design of a tensegrity-based artificial pectoral fin. International Journal of Solids and Structures, 48(22):3142-3159. 146

[Morison et al., 1950] Morison, J., Johnson, J., Schaaf, S., et al. (1950). The force exerted by surface waves on piles. Journal of Petroleum Technology, 2(05):149-154. 99

[Muijres et al., 2014] Muijres, F. T., Elzinga, M. J., Melis, J. M., and Dickinson, M. H. (2014). Flies evade looming targets by executing rapid visually directed banked turns. Science, 344(6180):172-177. 5, 6, 48, 67, 76, 89, 107

[Nabawy and Crowther, 2014] Nabawy, M. R. A. and Crowther, W. J. (2014). On the quasisteady aerodynamics of normal hovering flight part i: the induced power factor. Journal of The Royal Society Interface, 11(93). 15

[Nayfeh and Mook, 2008] Nayfeh, A. H. and Mook, D. T. (2008). Nonlinear oscillations. John Wiley \& Sons. 18

[Noca et al., 1997] Noca, F., Shiels, D., and Jeon, D. (1997). Measuring instantaneous fluid dynamic forces on bodies, using only velocity fields and their derivatives. Journal of Fluids and Structures, 11(3):345-350. 12

[OBERDORSTER and Grant, 2007] OBERDORSTER, U. and Grant, P. R. (2007). Predator foolhardiness and morphological evolution in 17-year cicadas (magicicada spp.). Biological Journal of the Linnean Society, 90(1):1-13. 47, 49 
[Osborne, 1951] Osborne, M. (1951). Aerodynamics of flapping flight with application to insects. Journal of Experimental Biology, 28(2):221-245. 14

[Pesavento and Wang, 2004] Pesavento, U. and Wang, Z. J. (2004). Falling paper: Navierstokes solutions, model of fluid forces, and center of mass elevation. Physical review letters, 93(14):144501. 15, 71, 99

[Pfau, 1986] Pfau, H. K. (1986). Untersuchungen zur Konstruktion, Funktion und Evolution des Flugapparates der Libellen (Insecta, Odonata). Nederlandse entomologische Vereniging. 20, 21

[Pringle, 1949] Pringle, J. (1949). The excitation and contraction of the flight muscles of insects. The Journal of physiology, 108(2):226-232. 20

[Ribak and Swallow, 2007] Ribak, G. and Swallow, J. G. (2007). Free flight maneuvers of stalkeyed flies: do eye-stalks affect aerial turning behavior? Journal of Comparative Physiology A, 193(10):1065-1079. 25

[Ristroph et al., 2010] Ristroph, L., Bergou, A. J., Ristroph, G., Coumes, K., Berman, G. J., Guckenheimer, J., Wang, Z. J., and Cohen, I. (2010). Discovering the flight autostabilizer of fruit flies by inducing aerial stumbles. Proceedings of the National Academy of Sciences, 107(11):4820-4824. 18, 116

[Ristroph et al., 2009] Ristroph, L., Berman, G. J., Bergou, A. J., Wang, Z. J., and Cohen, I. (2009). Automated hull reconstruction motion tracking applied to sideways maneuvers of freeflying insects. Journal of Experimental Biology, 212(9):1324-1335. 6, 25

[Ros et al., 2015] Ros, I. G., Badger, M. A., Pierson, A. N., Bassman, L. C., and Biewener, A. A. (2015). Pigeons produce aerodynamic torques through changes in wing trajectory during low speed aerial turns. The Journal of experimental biology, 218(3):480-490. 48, 86

[Ruppell, 1989] Ruppell, G. (1989). Kinematic analysis of symmetrical flight manoeuvres of odonata. Journal of Experimental Biology, 144(1):13-42. 19, 68, 117

[Sane and Dickinson, 2002] Sane, S. P. and Dickinson, M. H. (2002). The aerodynamic effects of wing rotation and a revised quasi-steady model of flapping flight. Journal of Experimental Biology, 205(8):1087-1096. 13, 15, 16, 19, 99, 112, 139

[Schilstra and Hateren, 1999] Schilstra, C. and Hateren, J. (1999). Blowfly flight and optic flow. i. thorax kinematics and flight dynamics. The journal of experimental biology, 202(11):1481-1490. 26

[Scruton et al., 1941] Scruton, C., Committee, A. R., et al. (1941). Some experimental determinations of the apparent additional mass effect for an aerofoil and for flat plates. HM Stationery Office. 11

[Sherman and Dickinson, 2003] Sherman, A. and Dickinson, M. H. (2003). A comparison of visual and haltere-mediated equilibrium reflexes in the fruit fly drosophila melanogaster. Journal of Experimental Biology, 206(2):295-302. 67

[Sherman and Dickinson, 2004] Sherman, A. and Dickinson, M. H. (2004). Summation of visual and mechanosensory feedback in drosophila flight control. Journal of Experimental Biology, 207(1):133-142. 67 
[Shih and Buchanan, 1971] Shih, C. C. and Buchanan, H. J. (1971). The drag on oscillating flat plates in liquids at low reynolds numbers. Journal of Fluid Mechanics, 48(02):229-239. 19

[Spedding and Maxworthy, 1986] Spedding, G. and Maxworthy, T. (1986). The generation of circulation and lift in a rigid two-dimensional fling. Journal of Fluid Mechanics, 165:247-272. 11

[Springthorpe et al., 2012] Springthorpe, D., Fernandez, M. J., and Hedrick, T. L. (2012). Neuromuscular control of free-flight yaw turns in the hawkmoth manduca sexta. The Journal of experimental biology, 215(10):1766-1774. 20, 21

[Sun and Xiong, 2005] Sun, M. and Xiong, Y. (2005). Dynamic flight stability of a hovering bumblebee. The Journal of experimental biology, 208(3):447-459. 18

[Tanaka et al., 2005] Tanaka, H., Hoshino, K., Matsumoto, K., and Shimoyama, I. (2005). Flight dynamics of a butterfly-type ornithopter. In Intelligent Robots and Systems, 2005.(IROS 2005). 2005 IEEE/RSJ International Conference on, pages 2706-2711. IEEE. 22

[Taylor et al., 2003] Taylor, G. K., Nudds, R. L., and Thomas, A. L. (2003). Flying and swimming animals cruise at a strouhal number tuned for high power efficiency. Nature, 425(6959):707-711. 10

[Taylor and Thomas, 2003] Taylor, G. K. and Thomas, A. L. (2003). Dynamic flight stability in the desert locust schistocerca gregaria. Journal of Experimental Biology, 206(16):2803-2829. 18, 30

[Taylor and Zbikowski, 2005] Taylor, G. K. and Zbikowski, R. (2005). Nonlinear time-periodic models of the longitudinal flight dynamics of desert locusts schistocerca gregaria. Journal of The Royal Society Interface, 2(3):197-221. 18

[Tiegs, 1955] Tiegs, O. (1955). The flight muscles of insects-their anatomy and histology; with some observations on the structure of striated muscle in general. Philosophical Transactions of the Royal Society of London. Series B, Biological Sciences, pages 221-348. 20

[Trimarchi and Schneiderman, 1995] Trimarchi, J. R. and Schneiderman, A. M. (1995). Different neural pathways coordinate drosophila flight initiations evoked by visual and olfactory stimuli. The Journal of experimental biology, 198(5):1099-1104. 67

[Tu and Dickinson, 1996] Tu, M. and Dickinson, M. (1996). The control of wing kinematics by two steering muscles of the blowfly (calliphora vicina). Journal of Comparative Physiology A, 178(6):813-830. 8, 20

[Van Den Berg and Ellington, 1997] Van Den Berg, C. and Ellington, C. P. (1997). The threedimensional leading-edge vortex of a 'hovering'model hawkmoth. Philosophical Transactions of the Royal Society of London. Series B: Biological Sciences, 352(1351):329-340. 11

[Vanella et al., 2009] Vanella, M., Fitzgerald, T., Preidikman, S., Balaras, E., and Balachandran, B. (2009). Influence of flexibility on the aerodynamic performance of a hovering wing. Journal of Experimental Biology, 212(1):95-105. 14

[Wagner, 1986] Wagner, H. (1986). Flight performance and visual control of flight of the freeflying housefly (musca domestica 1.) i. organization of the flight motor. Philosophical Transactions of the Royal Society B: Biological Sciences, 312(1158):527-551. 6, 25 
[Wakeling and Ellington, 1997] Wakeling, J. and Ellington, C. (1997). Dragonfly flight. ii. velocities, accelerations and kinematics of flapping flight. Journal of experimental biology, 200(3):557582. 19, 68, 117

[Wan et al., 2015] Wan, H., Dong, H., and Gai, K. (2015). Computational investigation of cicada aerodynamics in forward flight. Journal of The Royal Society Interface, 12(102):20141116. 13, $14,49,50,51,59$

[Wang et al., 2008] Wang, H., Ando, N., and Kanzaki, R. (2008). Active control of free flight manoeuvres in a hawkmoth, agrius convolvuli. Journal of Experimental Biology, 211(3):423-432. 20

[Wang et al., 2003] Wang, H., Zeng, L., Liu, H., and Yin, C. (2003). Measuring wing kinematics, flight trajectory and body attitude during forward flight and turning maneuvers in dragonflies. Journal of Experimental Biology, 206(4):745-757. 25, 28, 67, 127

[Wang et al., 2004] Wang, Z. J., Birch, J. M., and Dickinson, M. H. (2004). Unsteady forces and flows in low reynolds number hovering flight: two-dimensional computations vs robotic wing experiments. Journal of Experimental Biology, 207(3):449-460. 15

[Wang and Russell, 2007] Wang, Z. J. and Russell, D. (2007). Effect of forewing and hindwing interactions on aerodynamic forces and power in hovering dragonfly flight. Physical review letters, 99(14):148101. 13

[Whitney and Wood, 2010] Whitney, J. and Wood, R. (2010). Aeromechanics of passive rotation in flapping flight. Journal of Fluid Mechanics, 660:197-220. 11, 16, 95, 97, 99, 100, 105, 123

[Williams and Simon, 1995] Williams, K. S. and Simon, C. (1995). The ecology, behavior, and evolution of periodical cicadas. Annual review of entomology, 40(1):269-295. 49

[Willmott and Ellington, 1997] Willmott, A. P. and Ellington, C. P. (1997). The mechanics of flight in the hawkmoth manduca sexta. i. kinematics of hovering and forward flight. The Journal of Experimental Biology, 200(21):2705-2722. 8

[Willmott et al., 1997] Willmott, A. P., Ellington, C. P., and Thomas, A. L. (1997). Flow visualization and unsteady aerodynamics in the flight of the hawkmoth, manduca sexta. Philosophical Transactions of the Royal Society B: Biological Sciences, 352(1351):303-316. 11, 12

[Wolf, 1990] Wolf, H. (1990). On the function of a locust flight steering muscle and its inhibitory innervation. Journal of Experimental Biology, 150(1):55-80. 21

[Wood et al., 2012] Wood, R., Finio, B., Karpelson, M., Ma, K., Pérez-Arancibia, N., Sreetharan, P., Tanaka, H., and Whitney, J. (2012). Progress on 'pico' air vehicles. The International Journal of Robotics Research, 31(11):1292-1302. 23

[Wood, 2007] Wood, R. J. (2007). Design, fabrication, and analysis of a 3dof, 3cm flapping-wing mav. In Intelligent Robots and Systems, 200\%. IROS 200\%. IEEE/RSJ International Conference on, pages 1576-1581. IEEE. 22

[Wu and Sun, 2012] Wu, J. H. and Sun, M. (2012). Floquet stability analysis of the longitudinal dynamics of two hovering model insects. Journal of The Royal Society Interface, 9(74):20332046. 18 
[Zanker, 1988] Zanker, J. (1988). On the mechanism of speed and altitude control in drosophila melanogaster. Physiological entomology, 13(3):351-361. 62

[Zeyghami and Dong, 2012] Zeyghami, S. and Dong, H. (2012). A body reorientation strategy in insect takeoff flight. In 50th AIAA Aerospace Sciences Meeting including the New Horizons Forum and Aerospace Exposition, page 25. 30

[Zeyghami and Dong, 2015] Zeyghami, S. and Dong, H. (2015). Study of turning takeoff maneuver in free-flying dragonflies: effect of dynamic coupling. arXiv preprint arXiv:1502.06858. 30

[Zhang and Sun, 2010] Zhang, Y. and Sun, M. (2010). Dynamic flight stability of a hovering model insect: lateral motion. Acta Mechanica Sinica, 26(2):175-190. 18, 69

[Zhao et al., 2009] Zhao, L., Huang, Q., Deng, X., and Sane, S. P. (2009). Aerodynamic effects of flexibility in flapping wings. Journal of The Royal Society Interface, page rsif20090200. 14 